\title{
Geometria das hipersuperfícies de conflito
}

\author{
Plácido de Jesus da Silva Leitão Junior
}

\author{
DISSERTAÇÃO APRESENTADA \\ $\mathrm{AO}$ \\ INSTITUTO DE MATEMÁTICA E ESTATÍSTICA \\ DA \\ UNIVERSIDADE DE SÃO PAULO \\ PARA \\ OBTENÇÃO DO GRAU DE MESTRE \\ EM \\ MATEMÁTICA APLICADA
}

Orientador: Prof. Dr. Jorge Manuel Sotomayor Tello

Durante a elaboração deste trabalho o autor foi bolsista da FAPESP Processo 98/15064-2

São Paulo, 10 de dezembro de 2001 


\title{
Geometria das hipersuperfícies de Conflito
}

\author{
Este exemplar corresponde à redação final da \\ dissertação devidamente corrigida e defendida \\ por Plácido de Jesus da Silva Leitão Junior e \\ aprovada pela comissão julgadora.
}

São Paulo, 10 de dezembro de 2001.

Banca examinadora:

Prof. Dr. Jorge Sotomayor (IME-USP)

Prof. Dr. Plínio Simões (IME-USP)

Prof. Dr. Ronaldo Garcia (IME-UFG) 
Dedico este trabalho a meus pais Plácido de Jesus da Silva Leitão, Irene Moraes Leitão e a meus irmãos Mercedes, Camila e Diogo . 


\section{Resumo}

Dados dois conjuntos $A$ e $B$ no $\mathbb{R}^{n}$, estudaremos o conjunto $C(A, B)=\{x \in$ $\left.\mathbb{R}^{n} / d(x, A)=d(x, B)\right\}$, denominado conjunto de conflito de $A$ e $B$. Abordaremos o caso em que o conjunto de conflito é uma hipersuperfície de classe $C^{k}, k \geq 3$. No caso $n=3$ determinamos, para as superfícies de conflito, as curvaturas Gaussianas e Média, a localização dos pontos umbílicos e um resultado inicial sobre o tipo Darbouxiano destes pontos. A matriz da derivada da normal, $D N$, e as funções simétricas elementares das hipersuperfícies de conflito do $\mathbb{R}^{4}$ também são estabelecidos. Os resultados são expressos em função das direções e curvaturas principais dos bordos dos conjuntos $A$ e $B$ dados, os quais são supostos convexos. 


\section{Abstract}

Given two sets $A$ and $B$ in $\mathbb{R}^{n}$, we will study the set $C(A, B)=\left\{x \in \mathbb{R}^{n} / d(x, A)=\right.$ $d(x, B)\}$, called the conflict set of $A$ and $B$. We will consider the case in which the conflict set is a hypersurface of class $C^{k}, k \geq 3$. In the case $n=3$ we find, for conflict surfaces, the Gaussian and Mean curvatures, the location of umbilic points and an initial result concerning the Darbouxian type of these points. The matrix of the derivative, $D N$, of the normal, as well as the elementary symmetric functions of conflict hipersurface in $\mathbb{R}^{4}$ are also found. The results are expressed in terms of the principal directions and curvatures of the borders of the given sets $A$ and $B$, which are assumed to be convex. 


\section{Agradecimentos}

- Ao Prof. Jorge Sotomayor pela sua orientação, estando sempre disponível para o bom desenvolvimento do nosso trabalho.

- Ao Prof. Plínio Simões que por diversas vezes, atenciosamente, me indicou e emprestou livros. Além disso, contribuiu também na elaboração dos exemplos deste trabalho.

- Ao Prof. Geraldo Ávila por ter contribuído de forma significativa na minha formação acadêmica.

- A todos os meus familiares pelo amor e atenção dados a mim durante todo este tempo de luta.

- A Cássia.

- Ao meus amigos do 508: Glória, Jarlle, Ribamar, Eduardo, Wellington, Robson.

- Ao meu eterno e melhor amigo Wellington Barros, que apesar da distância sempre esteve comigo.

- A todos os meus amigos do IME-USP.

- Aos amigos ... 


\section{Índice}

Resumo

Abstract

iv

Agradecimentos

V

Introdução

1 Conjuntos de Conflito no $\mathbb{R}^{3} \quad 7$

1.1 Introdução . . . . . . . . . . . . . . . . . . . . . . 7

1.2 Superfícies de Conflito . . . . . . . . . . . . . . . . 8

1.3 Geometria do Conflito . . . . . . . . . . . . . . . . . . . 12

1.4 Pontos Umbílicos nas Superfícies de Conflito . . . . . . . . . . . . . 25

1.5 Exemplos de Conjuntos de Conflito . . . . . . . . . . . . . . . . 31

1.5.1 Curvas de Conflito . . . . . . . . . . . . . . . . . . 31

1.5.2 Superfícies de Conflito . . . . . . . . . . . . . 36

2 Conjuntos de Conflito no $\mathbb{R}^{4} \quad 42$

2.1 Introdução . . . . . . . . . . . . . . . . . . . . . . . . 42

2.2 Hipersuperfícies de Conflito do $\mathbb{R}^{4} \ldots \ldots$. . . . . . . . . . . . 43

2.3 Funções Simétricas Elementares . . . . . . . . . . . . . . . . . . . . 45

$\begin{array}{ll}\text { Referências Bibliográficas } & 54\end{array}$ 


\section{Introdução}

Considerando dois conjuntos $A_{1}$ e $A_{2}$ no espaço Euclidiano. Um problema clássico é o estudo do conjunto de pontos de igual distância de ambos, tal é o caso da reta e um ponto que leva à parábola. O objetivo do presente estudo é analisar algumas propriedades geométricas desses conjuntos, ditos de conflito.

Os conjuntos de igual distância no espaço Euclidiano são denominados por vários nomes: bissetores (em geometria computacional), conjuntos equidistântes, conjuntos de conflito da função distância (em teoria de singularidades), etc. Nós adotaremos a expressão $C\left(A_{1}, A_{2}\right)$ para denominar o conjunto de conflito de $A_{1}$ e $A_{2}$, em certas ocasiões omitiremos os conjuntos usando apenas $C$.

Baseamos nosso trabalho no artigo de Sotomayor, Garcia e Siersma [13] que estuda as curvaturas das superfícies de conflito, definidas por pares de conjuntos convexos no $\mathbb{R}^{3}$. Também foi útil o artigo de Siersma [11], que faz um estudo dos conjuntos de conflito no plano, mostrando suas propriedades de diferenciabilidade e obtendo uma expressão para curvatura da curva de conflito. Considerando o caso em que os conjuntos são convexos.

Ambos os artigos determinam a derivada da normal ao conjunto de conflito em função dos dados geométricos dos bordos dos conjuntos $A_{i}$. No caso do plano, a curvatura da curva de conflito é dada em função das curvaturas das curvas que determinam os bordos dos conjuntos $A_{i}$. No caso do espaço tridimensional a curvatura. Gaussiana, a curvatura Média e a localização dos pontos umbílicos na superfície são determinadas em função das direções principais e das curvaturas dos bordos $B_{i}=\partial A_{i}$. A hipótese de convexidade dos bordos é fundamental nos trabalhos acima e será adotada neste trabalho. Um resultado inicial relativo ao tipo Darbouxiano dos 
pontos umbílicos na superfície de conflito é também estabelecido (ver definição na seção 1.4).

Presumimos que o nosso leitor conheça os fundamentos de Geometria Diferencial e Equações Diferenciais Ordinárias. Tomamos como base para este estudo os livros de Carmo [1], Struik [18], Gray [5] e O'Neil [8] para a parte de Geometria Diferencial e Sotomayor [12] para a parte de EDO (Equações Diferenciais Ordinárias). Na seção 1.4, apresentaremos uma introdução do estudo do comportamento das linhas de curvatura na vizinhança de um ponto umbílico, como apresentado por Sotomayor e Gutiérrez em [16], [15], [14] e por Sotomayor e Garcia em [4]. Nessa mesma seção é feito, um estudo inicial do tipo genérico de pontos umbílicos que podem aparecer em um determinado ponto da superfície de conflito.

No Capítulo 1 estudaremos as superfícies de conflito no $\mathbb{R}^{3}$. Sejam dois conjuntos fechados $A_{1}$ e $A_{2}$ no espaço $\mathbb{R}^{3}$, o qual é munido pela orientação canônica e da distância:

$$
d(p, q)=|p-q|=\sqrt{\sum_{i=1}^{3}\left(p_{i}-q_{i}\right)^{2}}=\langle p-q, p-q\rangle^{\frac{1}{2}},
$$

onde $<$, $>$ é o produto interno Euclidiano em $\mathbb{R}^{3}$.

O conjunto, $C\left(A_{1}, A_{2}\right)$, dito de conflito entre $A_{1}$ e $A_{2}$ está definido por

$$
C\left(A_{1}, A_{2}\right)=\left\{p ; d\left(p, A_{1}\right)=d\left(p, A_{2}\right)\right\}
$$

onde $d(p, A)=\inf \{d(p, q) ; q \in A\}$.

Consideraremos conjuntos convexos fechados, não vazios, com seus interiores disjuntos e bordos regulares $B_{i}$ de classe $C^{k}, k \geq 2$, orientados por seus campos de vetores normais $N_{i}$, apontando para o interior de $A_{i}$.

Um dos resultados mais importantes deste capítulo é a determinação da matriz de $D N$, a derivada de $N$ a normal a superfície de conflito em um ponto $p$, em função dos dados geométricos dos $A_{i}$. Através deste resultado, podemos encontrar as funções simétricas elementares dos valores próprios, que nada mais são que as curvaturas Gaussiana e Média. Outros resultados importantes podem ser extraídos da matriz. Abaixo nos referimos a alguns resultados que obteremos.

Lembramos que as curvaturas principais $k_{1}^{i} \leq k_{2}^{i}$ de $B_{i}$ são os valores próprios de $-D N_{i}$, e que a condição de convexidade de $A_{i}$ é equivalente a dizer que ambas tem 
o mesmo sinal. A curvatura Gaussiana é definida por

$$
\mathcal{K}^{i}=k_{1}^{i} k_{2}^{i}=\operatorname{det}\left(-D N_{i}\right)
$$

isto implica que $\mathcal{K}^{i}>0$. Lembramos também, que a curvatura média de $B_{i}$ é dada por

$$
\mathcal{H}^{i}=\frac{1}{2} \operatorname{tra} \varsigma o\left(-D N_{i}\right)=\frac{1}{2}\left(k_{1}^{i}+k_{2}^{i}\right),
$$

A expressão $\mathcal{U}^{i}$, também chamada de curvatura reversa,

$$
\mathcal{U}^{i}=\frac{1}{2}\left(k_{1}^{i}-k_{2}^{i}\right)=\sqrt{\left(\mathcal{H}^{i}\right)^{2}-\mathcal{K}^{i}}
$$

cujos zeros localizam os pontos umbilicos da superfície, também aparecerá a seguir.

Através da matriz de $D N$, podemos obter várias propriedades geométricas da superfície de conflito. Mostraremos que as curvaturas Gaussiana e Média em um ponto $p \in C / M$ a uma distância $r$ dos conjuntos $A_{i}$, são dadas respectivamente por

$$
\begin{gathered}
\mathcal{K}=\left[\frac{1}{2}\left(\mathcal{K}^{1}(r)+\mathcal{K}^{2}(r)\right)-\mathcal{H}^{1}(r) \mathcal{H}^{2}(r)+\cos \left(2\left(\alpha_{2}-\alpha_{1}\right)\right) \mathcal{U}^{1}(r) \mathcal{U}^{2}(r)\right], \\
\mathcal{H}=\left(\frac{1+\operatorname{sen}^{2} \phi}{4 \operatorname{sen} \phi}\right)\left[\left(\mathcal{H}^{1}(r)-\mathcal{H}^{2}(r)\right)+\left(\frac{\cos ^{2} \phi}{1+\operatorname{sen} \phi}\right)\left(\cos \left(2 \alpha_{1}\right) \mathcal{U}^{1}(r)-\cos \left(2 \alpha_{2}\right) \mathcal{U}^{2}(r)\right)\right],
\end{gathered}
$$

onde $M$ é o conjunto dos pontos da superfície de conflito, no qual o campo $N_{1}+N_{2}$ é singular. A seguir definiremos os elementos que constituem as expressões acima.

Chamaremos de $\Pi_{i}$ a função que projeta um ponto $p$ da superfície de conflito no ponto $p_{i}$ de $B_{i}$, que realiza a distância mínima. Os ângulos $\alpha_{1}$ e $\alpha_{2}$ são os que as direções principais mínimas de $B_{1}$ e $B_{2}$, nos pontos $p_{1}$ e $p_{2}$ fazem com os vetores $D \Pi_{i}\left(T_{1}(p)\right)$, onde $T_{1}$ o vetor unitário na direção de $N_{1}+N_{2}$. O ângulo $\phi$ é a metade do ângulo entre $N_{1}$ e $N_{2}$.

Nas expressões acima, as curvaturas $\mathcal{K}^{i}(r)=\frac{\mathcal{K}^{i}}{1-2 r \mathcal{H}^{i}+r^{2} \mathcal{K}^{i}}, \mathcal{H}^{i}(r)=\frac{\mathcal{H}^{i}-r \mathcal{K}^{i}}{1-2 r \mathcal{H}^{i}+r^{2} \mathcal{K}^{i}}$, $\mathcal{U}^{i}(r)=\frac{\mathcal{U}^{i}}{1-2 r \mathcal{H}^{i}+r^{2} \mathcal{K}^{i}}$ são as das superfícies deslocadas'normalmente duma distância $r$ das $B_{i}$.

Estes resultados devidos a Sotomayor, Siersma e Garcia [13], estendem para superfícies o trabalho de Siersma [11], desenvolvido para curvas no plano. Damos a nossa versão de como obter o resultado do Siersma como uma decorrência dos resultados tratados neste capítulo. 


\section{Introdução}

Outro resultado obtido estabelece que um ponto $p \in C$ é umbílico se e somente se $\Upsilon=\Psi=0$, onde

$$
\begin{gathered}
\Upsilon=\mathcal{U}^{2} \operatorname{sen}\left(2 \alpha_{2}\right)-\mathcal{U}^{1} \operatorname{sen}\left(2 \alpha_{1}\right) \\
\Psi=\left(\frac{3-\cos 2 \phi}{2}\right)\left\{\left[\mathcal{H}^{1}-\mathcal{H}^{2}\right]\left(\frac{1+\cos 2 \phi}{3-\cos 2 \phi}\right)+\left[\mathcal{U}^{1} \cos \left(2 \alpha_{1}\right)-\mathcal{U}^{2} \cos \left(2 \alpha_{2}\right)\right]\right\} .
\end{gathered}
$$

Também foi provado que num ponto não umbílico $p \in C$ as direções principais são caracterizadas por fazerem um ângulo $\theta \operatorname{com} T_{1}$, onde $\theta$ verifica:

$$
\tan 2 \theta=\frac{2 \Upsilon \operatorname{sen} \theta}{\Psi}
$$

Isto é equivalente a dar, em linguagem geométrica, as equações diferenciais das linhas de curvatura principal nas superfícies de conflito.

Encontramos uma expressão para a curvatura normal à superfície de conflito, em $p \in C / M$ na direção de um vetor unitário $X$ tangente a $C / M$ em $p$, dada por

$$
\begin{aligned}
k_{n}(X)=-\left\{\frac{\cos ^{2}(\theta) \operatorname{sen} \phi}{2}\left[k_{n}^{2}(r)-k_{n}^{1}(r)\right]+\right. & \frac{\operatorname{sen}^{2}(\theta)}{2 \operatorname{sen} \phi}\left[k_{n \perp}^{2}(r)-k_{n \perp}^{1}(r)\right] \\
& \left.+\cos (\theta) \operatorname{sen}(\theta)\left[\tau_{g}^{2}(r)-\tau_{g}^{1}(r)\right]\right\},
\end{aligned}
$$

onde $\theta$ é o ângulo entre $\mathrm{X}$ e $T_{1}$. Igualando esta expressão a zero, conseguimos uma forma equivalente as equações diferenciais para as linhas assintóticas.

Faremos uma breve introdução sobre como caracterizar o tipo de ponto umbílico genérico que pode aparecer em uma superfície. Condições algébricas serão impostas ao 3-jato da superfície para que tenhamos o tipo Darbouxiano do ponto umbílico. Com o intuito de caracterizarmos o tipo umbílico que pode aparecer no ponto $p_{m} \in$ $M$, calculamos o 3-jato da superfície de conflito neste ponto, em função dos jatos dos bordos $B_{i}$.

Por fim daremos alguns exemplos de curvas e superfícies de conflito com a ajuda do programa Mathematica 4.

No Capítulo 2 estudaremos as hipersuperfícies de conflito no $\mathbb{R}^{4}$. Sejam dois conjuntos fechados $A_{1}$ e $A_{2}$ no espaço $\mathbb{R}^{4}$. As definições que usaremos neste capítulo são as mesmas que introduzimos no capítulo anterior, com as devidas adaptações para o $\mathbb{R}^{4}$.

Para que tenhamos uma boa regularidade nas hipersuperfícies de conflito consideraremos somente o caso em que os $A_{i}$ são conjuntos convexos fechados, não vazios, 
com seus interiores disjuntos e bordos regulares $B_{i}=\partial A_{i}$ de classe $C^{k}, k \geq 2$, orientados por seus campos de vetores normais $N_{i}$, apontando para o interior de $A_{i}$.

Faremos um estudo semelhante ao do capítulo 1, com o propósito de obtermos uma expressão para a matriz de $D N$, a derivada da normal a hipersuperfície de conflito gerada pelos dois conjuntos dados $A_{1}$ e $A_{2}$. Na proposição 2, a matriz será dada em função dos dados geométricos dos $B_{i}$, chegando à seguinte representação para $D N$ em um ponto $p \in C \backslash M$ em relação a base $\left\{T_{1}, T_{2}, T_{3}\right\}$ do hiperplano tangente,

$$
D N=\left(\begin{array}{ccc}
\frac{\operatorname{sen} \phi}{2}\left[k_{n_{1}}^{2}(r)-k_{n_{1}}^{1}(r)\right] & \frac{1}{2}\left[\tau_{12}^{2}(r)-\tau_{12}^{1}(r)\right] & \frac{1}{2}\left[\tau_{13}^{2}(r)-\tau_{13}^{1}(r)\right] \\
\frac{1}{2}\left[\tau_{12}^{2}(r)-\tau_{12}^{1}(r)\right] & \frac{1}{2 \operatorname{sen} \phi}\left[k_{n_{2}}^{2}(r)-k_{n_{2}}^{1}(r)\right] & \frac{1}{2 \operatorname{sen} \phi}\left[\tau_{23}^{2}(r)-\tau_{23}^{1}(r)\right] \\
\frac{1}{2}\left[\tau_{13}^{2}(r)-\tau_{13}^{1}(r)\right] & \frac{1}{2 \operatorname{sen} \phi}\left[\tau_{23}^{2}(r)-\tau_{23}^{1}(r)\right] & \frac{1}{2 \operatorname{sen} \phi}\left[k_{n_{3}}^{2}(r)-k_{n_{3}}^{1}(r)\right]
\end{array}\right)
$$

onde $M$ é o conjunto dos pontos onde o campo $N_{1}+N_{2}=0$ e os objetos que aparecem na matriz acima estão em função das curvaturas e direções principais dos $B_{i}$.

Lembramos que as curvaturas principais $k_{1}^{i} \leq k_{2}^{i} \leq k_{3}^{i}$ de $B_{i}$ são os valores próprios de $-D N_{i}$, e que a condição de convexidade de $A_{i}$ é equivalente a dizer que todas possuem o mesmo sinal. As funções simétricas elementares das hipersuperfícies $B_{i}$ são obtidas através dos autovalores da matriz $D N_{i}$. e suas expressões são dadas por

$$
\begin{gathered}
\mathcal{K}^{i}=k_{1}^{i} k_{2}^{i} k_{3}^{i}=\operatorname{det}\left(-D N_{i}\right), \\
\mathcal{H}_{1}^{i}=\frac{1}{2}\left(k_{1}^{i} k_{2}^{i}+k_{1}^{i} k_{3}^{i}+k_{2}^{i} k_{3}^{i}\right) . \\
\mathcal{H}^{i}=\frac{1}{3}\left(k_{1}^{i}+k_{2}^{i}+k_{3}^{i}\right)=\frac{1}{3} \operatorname{tra} \varsigma\left(-D N_{i}\right),
\end{gathered}
$$

De acordo com a notação introduzida por Forsyth [3] (p.40 seção 281 capítulo XVI) temos que $\mathcal{H}^{i}$ é chamada por curvatura linear, $\mathcal{H}_{1}^{i}$ de curvatura superficial e $\mathcal{K}^{i}$ a curvatura espacial (ou curvatura volumétrica).

A importância de determinarmos a matriz $D N$ em função dos dados dos $B_{i}$ é que nos permite também encontrar as funções simétricas elementares da hipersuperfície de conflito em função dos objetos geométricos dos bordos dos conjuntos $A_{i}$. Obtemos as seguintes expressões para as funções simétricas elementares de $C$ :

$$
\begin{gathered}
\mathcal{K}=\operatorname{det}(-D N)=-\left[k_{n_{1}} k_{n_{2}} k_{n_{3}}+2 \tau_{12} \tau_{13} \tau_{23}-k_{n_{1}}\left(\tau_{23}\right)^{2}-k_{n_{2}}\left(\tau_{13}\right)^{2}-k_{n_{3}}\left(\tau_{12}\right)^{2}\right], \\
\mathcal{H}=\operatorname{traço~}(-D N)=-\frac{1}{3}\left(k_{n_{1}}+k_{n_{2}}+k_{n_{3}}\right), \\
\mathcal{H}_{1}=\frac{1}{2}\left\{\left[k_{n_{1}} k_{n_{2}}-\left(\tau_{12}\right)^{2}\right]+\left[k_{n_{1}} k_{n_{3}}-\left(\tau_{13}\right)^{2}\right]+\left[k_{n_{2}} k_{n_{3}}-\left(\tau_{23}\right)^{2}\right]\right\},
\end{gathered}
$$


lembramos que como $D N$ esta dada em função das curvaturas e direções principais de $B_{i}$, as expressões acima também estão.

Este é o resultado do estudo desenvolvido sobre as expressões das funções simétricas elementares da hipersuperfície de conflito, com a intenção de expressá-las em função das funções simétricas elementares dos $B_{i}$, como foi feito para o $\mathbb{R}^{3}$. Este estudo esta ainda em fase inicial. 


\section{Conjuntos de Conflito no $\mathbb{R}^{3}$}

\subsection{Introdução}

Neste capítulo serão estudados as superfícies de conflito do $\mathbb{R}^{3}$.

Dados dois conjuntos $A_{1}$ e $A_{2}$ no $\mathbb{R}^{3}$, definiremos o conjunto de conflito, $C\left(A_{1}, A_{2}\right)$, como sendo o conjunto dos pontos, que estão a uma mesma distância dos dois conjuntos dados.

Para o desenvolvimento deste capítulo, nos baseamos no trabalho de Sotomayor, Garcia e Siersma [13], na qual foi feita uma extensão dos resultados de Siersma [11] para curvas de conflito planas.

Trataremos o caso em que o conjunto de conflito é uma superfície regular de classe $C^{k}, k \geq 1$. A regularidade é obtida ao considerarmos conjuntos fechados, não vazios, com seus interiores disjuntos e bordos regulares de classe $C^{k}, k \geq 2$.

Um dos resultados mais importantes deste capítulo, é a determinação da matriz de $D N$, a derivada de $N$ a normal a superfície de conflito em um ponto $p$, em função dos dados geométricos dos $A_{i}$. Através deste resultado, podemos encontrar as funções simétricas elementares, que são as curvaturas Gaussiana e Média.

Determinaremos outros objetos geométricos das superfícies de conflito tais como: localização dos pontos umbílicos e direções principais, curvatura normal e direções assintóticas. Estes resultados saem diretamente da matriz de $D N$.

No trabalho de Siersma [11], é obtida no plano $\mathbb{R}^{2}$, a expressão para a curvatura da curva de conflito em função das curvaturas dos bordos dos conjuntos que à define. 
Damos nossa interpretação, de como obter os resultados do Siersma através dos dados da matriz $D N$, fazendo uma restrição dos objetos introduzidos para curvas sobre a superfície de conflito.

Faremos uma introdução sobre o comportamento das linhas de curvatura na vizinhança de um ponto umbílico. Daremos algumas condições sobre o 3-jato das superfícies com o intuito de classificar os tipos genéricos de pontos umbílicos, que podem surgir. Para termos essa caracterização dos pontos umbílicos sobre as superfícies de conflito, acharemos uma expressão para o 3-jato da superfície de conflito em um ponto $p \in M$.

Por fim, daremos alguns exemplos de curvas e superfícies de conflito.

\subsection{Superfícies de Conflito}

Sejam $A_{1}$ e $A_{2}$ dois conjuntos não vazios, fechados no espaço Euclidiano $\mathbb{R}^{3}$. Adotaremos a orientação canônica do $\mathbb{R}^{3}$, através da seguinte classe de equivalência: Diremos que duas bases $\mathrm{E}=\left\{e_{1}, e_{2}, e_{3}\right\}$, base canônica, e $\mathrm{F}=\left\{f_{1}, f_{2}, f_{3}\right\}$ são igualmente orientadas, quando a matriz de passagem de $\mathrm{E}$ para $\mathrm{F}$ tiver determinante positivo.

A distância que utilizaremos é dada por

$$
d(p, q)=|p-q|=\sqrt{\sum_{i=1}^{3}\left(p_{i}-q_{i}\right)^{2}}=\langle p-q, p-q\rangle^{\frac{1}{2}}
$$

onde $p=\left(p_{1}, p_{2}, p_{3}\right), q=\left(q_{1}, q_{2}, q_{3}\right)$ e $\langle$.$\rangle é o produto interno Euclidiano usual,$ expresso da seguinte forma: sejam $w$ e $v \in \mathbb{R}^{3}$, temos que

$$
\langle w, v\rangle=w_{1} v_{1}+w_{2} v_{2}+w_{3} v_{3}
$$

onde $w=\left(w_{1}, w_{2}, w_{3}\right)$ e $v=\left(v_{1}, v_{2}, v_{3}\right)$.

O conjunto de conflito $C\left(A_{1}, A_{2}\right)$ entre $A_{1}$ e $A_{2}$ é definido por

$$
C\left(A_{1}, A_{2}\right)=\left\{p \in \mathbb{R}^{3} ; d\left(p, A_{1}\right)=d\left(p, A_{2}\right)\right\},
$$

onde $d(p, A)=\inf \{d(p, q) ; q \in A\}$.

O conjunto $C\left(A_{1}, A_{2}\right)$ é também visto como o bordo comum entre o território de $A_{1}$ relativo a $A_{2}$, definido por 


$$
\operatorname{Terr}\left(A_{1}, A_{2}\right)=\left\{p ; d\left(p, A_{1}\right)<d\left(p, A_{2}\right)\right\},
$$

e $\operatorname{Terr}\left(A_{2}, A_{1}\right)$, que é o território de $A_{2}$ relativo a $A_{1}$.

O conjunto de conflito também é chamado de bissetor na Geometria Computacional e de conjunto equidistante entre $A_{1}$ e $A_{2}$.

Em todo este capítulo consideraremos somente o caso onde os $A_{i}$ são conjuntos fechados, convexos, com interiores disjuntos e bordos regulares $B_{i}=\partial A_{i}$, de classe $C^{k}, k \geq 2$.

Os bordos $B_{i}$ são orientados, ou seja, existem campos diferenciáveis de vetores unitários $N_{i}: B_{i} \rightarrow \mathbb{R}^{3}$ que associam para cada $p \in B_{i}$ um vetor normal unitário $N_{i}(p) \in \mathbb{R}^{3}$ para $B_{i}$ em $p$. A convenção adotada será que $N_{i}$ estará sempre apontando para o interior de $A_{i}$.

Para cada $p \in B_{i}$ podemos escolher, na linha normal passando por $p$, um intervalo aberto $I_{p}$ próximo de $p$ de comprimento, digamos, $2 \epsilon_{p}\left(\epsilon_{p}\right.$ variando com $\left.p\right)$ de modo que se $p \neq q \in B_{i}$, então $I_{p} \cap I_{q}=\varnothing$. A união $\bigcup I_{p}, p \in B_{i}$, constitui um conjunto aberto $V_{i}$ de $\mathbb{R}^{3}$, que contém $B_{i}$ e tem a propriedade que por cada ponto de $V_{i}$ passa uma única reta normal a $B_{i}$. Denominamos $V_{i}$ de vizinhança tubular de $B_{i}$. Como os $B_{i}$, por hipótese, são convexos, podemos garantir que este processo pode ser aplicado para $\epsilon$ qualquer na direção de $-N_{i}$, já que $N_{i}$ esta apontando para o interior de $B_{i}$. O mesmo não pode ser feito para $\epsilon$ qualquer na direção da normal, já que pela hipótese das superfícies serem convexas, nesta direção as normais vizinhas se intersectam ao longo do conjunto focal. ( M. Carmo [1], p. 110, seção 2-7)

Denotaremos por $A_{i}^{r}$ o conjunto dos pontos a uma distância $r \geq 0$ de $A_{i}$; seu bordo é a superfície $B_{i}^{r}$ obtida pelo deslocamento de cada ponto $p_{i}$ de $B_{i}$ pela função $\sum_{i}^{r}\left(p_{i}\right)=p_{i}-r N_{i}\left(p_{i}\right)$. De acordo com as propriedades acima, de vizinhança tubular, temos que $\Sigma_{i}^{r}$ é um difeomorfismo de classe $C^{k-1}$, cuja inversa, definida no conjunto fechado $E_{i}=\mathbb{R}^{3} \backslash \operatorname{Int}\left(A_{i}\right)$, associa as projeções $\Pi_{i}(p)=p_{i}$ nos $B_{i}$, caracterizadas por

$$
d\left(p, A_{i}\right)=\left\langle\Pi_{i}(p)-p, N_{i}\left(\Pi_{i}(p)\right)\right\rangle,
$$

estas funções $\Pi_{i}$ serão úteis nos cálculos a seguir, restringindo o seu domínio ao conjunto de conflito, no qual estamos interessados. Podemos também definir a projeção $\Pi_{i}^{r}$, através da seguinte composição $\Pi_{i}^{r}=\Sigma_{i}^{r}$ o $\Pi_{i}$, que levará um ponto de $E_{i}$ para $B_{i}^{r}$. 
Além disso, $C\left(A_{1}, A_{2}\right)$ é definido implicitamente como o conjunto de nível zero da função

$$
c(p)=d\left(p, A_{2}\right)-d\left(p, A_{1}\right),
$$

que é regular de classe $C^{k-1}$, isto se verifica facilmente pela definição que demos acima da função $\Pi_{i}$. Calculando o gradiente dessa função temos

$$
\nabla c(p)=N_{1}\left(\Pi_{1}(p)\right)-N_{2}\left(\Pi_{2}(p)\right)
$$

Neste trabalho, a superfície de conflito $C$ será orientada pela normal unitária $N$, ao longo do $\nabla c$ e apontando de $A_{2}$ para $A_{1}$ :

$$
N=\left|N_{1}\left(\Pi_{1}(p)\right)-N_{2}\left(\Pi_{2}(p)\right)\right|^{-1}\left[N_{1}\left(\Pi_{1}(p)\right)-N_{2}\left(\Pi_{2}(p)\right)\right]
$$

Para simplificar a notação escreveremos $\nu(V)=|V|^{-1} V$ para denotar a normali$z a c ̧ a \tilde{o} o$ de um vetor não nulo $V$. Logo

$$
N=\nu\left(N_{1}\left(\Pi_{1}(p)\right)-N_{2}\left(\Pi_{2}(p)\right)\right)
$$

Um triedro móvel positivo definido em $C$ é dado por $\left\{T_{1}, T_{2}, N\right\}$, onde:

$$
\begin{gathered}
T_{1}=\nu\left(N_{1}\left(\Pi_{1}(p)\right)+N_{2}\left(\Pi_{2}(p)\right)\right), \\
T_{2}=N_{1}\left(\Pi_{1}(p)\right) \wedge N_{2}\left(\Pi_{2}(p)\right) .
\end{gathered}
$$

Note que o campo de vetores $T_{i}$ são singulares nos pontos $p$ do conjunto fechado $M=M\left(A_{1}, A_{2}\right)$, onde $N_{1}\left(\Pi_{1}(p)\right)+N_{2}\left(\Pi_{2}(p)\right)=0$, que ocorre quando a distância de $C$ para $A_{i}$,

$$
r(p)=d\left(p, A_{1}\right)=d\left(p, A_{2}\right),
$$

é mínima, assumindo o valor $d_{m}=\frac{1}{2} d\left(A_{1}, A_{2}\right)$. A condição de convexidade estrita dos $B_{i}$ nos conjuntos $\Pi_{i}(M)$, reduz $M$ a um único ponto $p_{m}$.

Observamos também que $T_{2}$ pode ser definido como o vetor unitário obtido da intersecção dos planos tangentes de $B_{i}^{r}$ em $p$, de tal forma que a base fique positiva. Isso é óbvio, pois se temos um vetor $v$ na intersecção dos planos tangente a $B_{i}^{r} \mathrm{em}$ $p$, isso implica que $v$ é normal a $N_{1}$ e a $N_{2}$. Portanto $v$ é paralelo a $T_{2}$. Na figura abaixo temos um esquema disto, onde $T C$ é o espaço tangente em $p \in C \backslash M$ e $T B_{i}^{r}$ são os espaços tangentes a $p_{i}=p=\Pi_{i}^{r}(p) \in B_{i}^{r}$. 


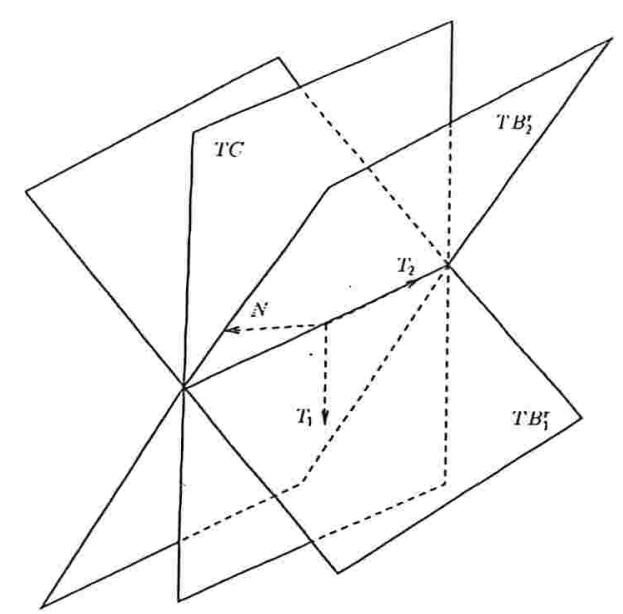

Figura 1.1: Triedro $\left\{T_{1}, T_{2}, N\right\}$

Podemos dividir a superfície de conflito nos pontos onde a intersecção do planos tangentes de $B_{i}^{r}$ em $p \in C$ é gerada por um vetor, neste caso, estamos em $C \backslash M$. Já quando é gerada por dois vetores linearmente independentes, estamos em $M$.

Considerando a convexidade estrita de $B_{i}$ em $p_{i}$, implica que $D N_{i}$ é um automorfismo do espaço tangente $T B_{i}$, com a identificação usual do espaço tangente $T B_{i}$ em $p_{i}$ com o da esfera unitária $T S^{2}$, em $N_{i}\left(p_{i}\right)$. Em termos das curvaturas principais $k_{1}^{i} \leq k_{2}^{i}$, que são os autovalores de $-D N_{i}$, a condição de convexidade estrita e a orientação do campo normal implica que $0<k_{1}^{i} \leq k_{2}^{i}$ (Rodrigues [10], Cap. 3, pag. 30). Em termos da curvatura Gaussiana

$$
\mathcal{K}^{i}=k_{1}^{i} k_{2}^{i}=\operatorname{det}\left(-D N_{i}\right)
$$

isto implica que $\mathcal{K}^{i}>0$. Lembramos também que a curvatura média de $B_{i}$ é dada por

$$
\mathcal{H}^{i}=\frac{1}{2} \operatorname{traço}\left(-D N_{i}\right)=\frac{1}{2}\left(k_{1}^{i}+k_{2}^{i}\right),
$$

A expressão

$$
\mathcal{U}^{i}=\frac{1}{2}\left(k_{1}^{i}-k_{2}^{i}\right)=\sqrt{\left(\mathcal{H}^{i}\right)^{2}-\mathcal{K}^{i}}
$$

também chamada de curvatura reversa, cujos zeros localizam os pontos umbiticos da superfície, será também usada neste trabalho. 


\subsection{Geometria do Conflito}

Seja $p \in C \backslash M$ tal que $p_{i}=\Pi_{i}(p)$ não sejam pontos umbílicos de $B_{i}$. Seja $\left\{E_{1}^{i}, E_{2}^{i}, N_{i}\right\}$ o triedro móvel positivo principal em $B_{i}$, numa vizinhança de $p_{i}$. Isto implica que

$$
D N_{i} \cdot E_{j}^{i}=-k_{j}^{i} E_{j}^{i}
$$

onde $0<k_{1}^{i}<k_{2}^{i}, i=1,2$, são as curvaturas principais de $B_{i}$. Denotemos por $\alpha_{i}$ o ângulo entre os vetores $E_{1}^{i}$ e $F_{1}^{i}=\nu\left(D \Pi_{i}(p) \cdot T_{1}\right)$. Escrevemos

$$
\tau_{g}^{i}=\tau_{g}^{i}\left(\alpha_{i}\right)=\left(k_{2}^{i}-k_{1}^{i}\right) \operatorname{sen}\left(\alpha_{i}\right) \cos \left(\alpha_{i}\right)
$$

para a torção geodésica na direção do vetor unitário $F_{1}^{i}$ de $B_{i}$. Escrevemos

$$
k_{n}^{i}=k_{n}^{i}\left(\alpha_{i}\right)=k_{1}^{i} \cos ^{2}\left(\alpha_{i}\right)+k_{2}^{i} \operatorname{sen}^{2}\left(\alpha_{i}\right)
$$

que pela fórmula de Euler é a curvatura normal de $B_{i}$ na direção de $F_{1}^{i}=\nu\left(D \Pi_{i}(p) \cdot T_{1}\right)$.

Similarmente, a curvatura normal de $B_{i}$ na direção de $F_{2}^{i}=\nu\left(D \Pi_{i}(p) \cdot T_{2}\right)$ é dada por

$$
k_{n \perp}^{i}=k_{n \perp}^{i}\left(\alpha_{i}\right)=k_{n}^{i}\left(\alpha_{i}+\frac{\pi}{2}\right)=k_{1}^{i} \operatorname{sen}^{2}\left(\alpha_{i}\right)+k_{2}^{i} \cos ^{2}\left(\alpha_{i}\right),
$$

Mostraremos algumas propriedades geométricas de $F_{1}^{i}$ e $F_{2}^{i}$ que serão úteis no decorrer deste capítulo.

A figura 1.2, a seguir, mostra um esquema unidimensional de como os vetores $F_{1}^{i}$ e $T B_{i}^{r}$, os planos tangentes a $B_{i}^{r}$, ficam dispostos em um ponto de $p \in C \backslash M$.

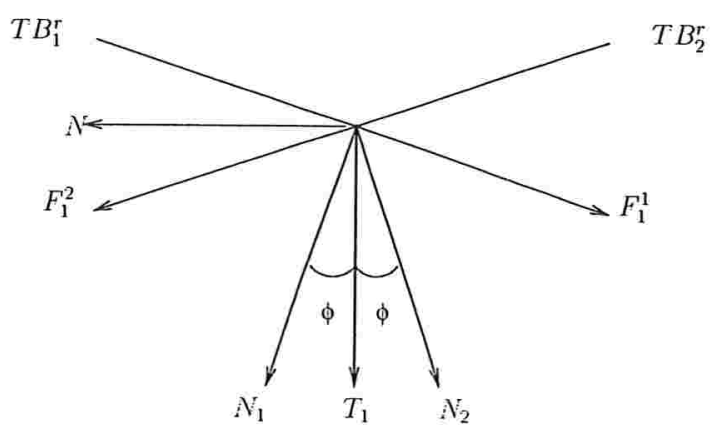

Figura 1.2: Seção plana no ponto p 
Lema 1 Denotemos por $\phi$ o ângulo entre $N_{i}$ e $T_{1}$. Então

$$
\operatorname{sen} \phi=\frac{1}{2}\left|N_{1}\left(\Pi_{1}(p)\right)-N_{2}\left(\Pi_{2}(p)\right)\right|=\left\langle F_{1}^{1}, T_{1}\right\rangle=\left\langle F_{1}^{2}, T_{1}\right\rangle
$$

Prova. Observemos que

$$
\begin{aligned}
2 \operatorname{sen}^{2} \phi & =1-\cos 2 \phi=1-\left\langle N_{1}, N_{2}\right\rangle \\
& =\frac{2-2\left\langle N_{1}, N_{2}\right\rangle}{2}=\frac{\left\langle N_{1}, N_{1}\right\rangle+\left\langle N_{2}, N_{2}\right\rangle-2\left\langle N_{1}, N_{2}\right\rangle}{2} \\
& =\frac{1}{2}\left\langle N_{1}-N_{2}, N_{1}-N_{2}\right\rangle=\frac{\left|N_{1}-N_{2}\right|^{2}}{2} .
\end{aligned}
$$

Então

$$
\operatorname{sen} \phi=\frac{1}{2}\left|N_{1}\left(\Pi_{1}(p)\right)-N_{2}\left(\Pi_{2}(p)\right)\right|
$$

Temos que $T_{1}$ pode ser escrito como combinação linear de $N_{i}$ e $F_{1}^{i}$, como mostra a figura 1.2. Logo $\left\langle F_{1}^{i}, T_{1}\right\rangle=\left\langle F_{1}^{i}, \cos \phi N_{i}+\operatorname{sen} \phi F_{1}^{i}\right\rangle=\operatorname{sen} \phi$.

Lema $2 A$ base $\left\{T_{1}, T_{2}\right\}$ em $C \backslash M$ é projetada pela derivada de $\Pi_{i}$ na base ortonormal $\left\{F_{1}^{i}, F_{2}^{i}\right\}$ em $B_{i}$.

Prova. Isto é visto facilmente através das propriedades da função projeção. Temos que $T_{2}$ pertence tanto ao plano tangente de $C \backslash M$ como a $T B_{i}^{r}$ (ver figura 1.1), logo $F_{2}^{i}$ será paralelo a $T_{2}$. Já que $T_{2}$ é ortogonal ao plano gerado por $N$ e $T_{1}$, que contém $F_{1}^{i}$, concluímos então que $\left\{F_{1}^{i}, F_{2}^{i}\right\}, i=1,2$, são bases ortonormais.

Lema 3 Os vetores $\left\{F_{1}^{i}, F_{2}^{i}\right\}, i=1$,2, podem ser escritos da seguinte forma

$$
\begin{gathered}
\left\{F_{1}^{1}=\nu\left(N_{2}-\left\langle N_{1}, N_{2}\right\rangle N_{1}\right), F_{2}^{1}=T_{2}\right\}, \\
\left\{F_{1}^{2}=\nu\left(-N_{1}+\left\langle N_{1}, N_{2}\right\rangle N_{2}\right), F_{2}^{2}=T_{2}\right\} .
\end{gathered}
$$

Prova. Para verificarmos a primeira afirmativa, basta escrever $N_{2}$ como combinação linear de $F_{1}^{1}$ e $N_{1}$ (ver figura 1.2), ou seja,

$$
\begin{aligned}
& N_{2}=\left\langle N_{1}, N_{2}\right\rangle N_{1}+\left\langle F_{1}^{1}, N_{2}\right\rangle F_{1}^{1} \Rightarrow\left\langle F_{1}^{1}, N_{2}\right\rangle F_{1}^{1}=N_{2}-\left\langle N_{1}, N_{2}\right\rangle N_{1} \\
& \Rightarrow \nu\left(\left\langle F_{1}^{1}, N_{2}\right\rangle F_{1}^{1}\right)=\nu\left(N_{2}-\left\langle N_{1}, N_{2}\right\rangle N_{1}\right) \Rightarrow F_{1}^{1}=\nu\left(N_{2}-\left\langle N_{1}, N_{2}\right\rangle N_{1}\right)
\end{aligned}
$$

utilizando-se da notação vetorial $F_{2}^{1}=T_{2}$, já que possuem a mesma direção e comprimento. Analogamente podemos mostrar a segunda afirmação. 
Ao fazermos o deslocamento paralelo na direção da normal das superfícies $B_{i}$ até termos $B_{i}^{r}$, o vetor $D \Pi_{i}^{r}\left(T_{1}\right)$ é dado por $D \Pi_{i}^{r}\left(T_{1}\right)=\operatorname{sen} \phi F_{1}^{i}$. Para ver esta afirmação basta tomar uma curva $\gamma(t) \subset C$ tal que $\gamma(0)=p, \gamma(0)=T_{1}$ e projetá-la sobre $B_{1}^{r}$. Fazendo a projeção teremos uma curva $\gamma_{1}^{r}(t)$ tal que $\gamma_{1}^{r}(0)=p_{1}=p$ e $\left(\gamma_{1}^{r}\right)^{\prime}(0)=$ $D \Pi_{1}^{r}\left(T_{1}\right)$. Com o auxílio da figura 1.3 podemos concluir que $\left(\gamma_{1}^{r}\right)^{\prime}(0)=\operatorname{sen} \phi F_{1}^{1}$. Observamos que este argumento pode ser utilizado para $i=2$.

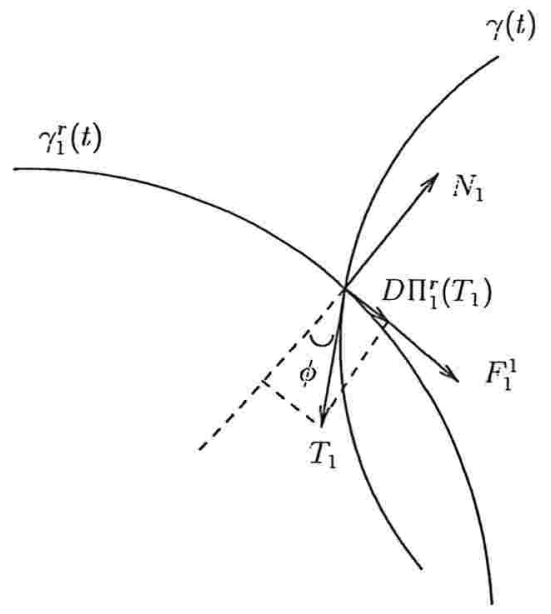

Figura 1.3: Derivada da Projeção

Os triedros principais $\left\{E_{1}^{i}, E_{2}^{i}, N_{i}\right\}$ em $B_{i}$ são transladados ao longo da normal para o triedro principal em $B_{i}^{r}$. Isto segue do fato que $\Sigma_{i}^{r}$ preserva os campos de direções principais assim como os pontos umbílicos. As curvaturas principais sofrem mudanças para $k_{j}^{i}(r)=\frac{k_{j}^{i}}{1-r k_{j}^{i}}$. Provaremos a seguir este fato.

Lema 4 Seja $\Sigma_{i}^{r}: B_{i} \rightarrow B_{i}^{r}$. Então para $X$ em $T B_{i}\left(p_{i}\right), D \Sigma_{i}^{r}(X)=X+r D N_{i}(X)$, $D N_{i}^{r}\left(D \Sigma_{i}^{r}(X)\right)=D N_{i}(X), \Sigma_{i}^{r}$ preserva as direções principais e umbilicos. Se $k_{j}^{i}$ é a curvatura principal de $B_{i}$ em $p_{i}$ na direção $E_{j}^{i}$, entãa $k_{j}^{i}(r)=\frac{k_{j}^{i}}{1-r k_{j}^{i}}$ é a correspondente curvatura principal de $B_{i}^{r}$ em $\sum_{i}^{r}\left(p_{i}\right)$ na direção $D \sum_{i}^{r}\left(E_{j}^{i}\right)$. Além disso, temos que as curvaturas Gaussiana e Média de $B_{i}^{r}$ são dadas respectivamente por:

$$
\begin{aligned}
\mathcal{K}^{i}(r) & =\frac{\mathcal{K}^{i}}{1-2 r \mathcal{H}^{i}+r^{2} \mathcal{K}^{i}}, \\
\mathcal{H}^{i}(r) & =\frac{\mathcal{H}^{i}-r \mathcal{K}^{i}}{1-2 r \mathcal{H}^{i}+r^{2} \mathcal{K}^{i}},
\end{aligned}
$$

onde $\mathcal{K}^{i}$ e $\mathcal{H}^{i}$ denotam as curvaturas Gaussiana e Média de $B_{i}$. 
Prova. Suponhamos que a normal $N_{i}$ seja dada por $\left(a_{1}, a_{2}, a_{3}\right)$ onde cada $a_{i}$ é de classe $C^{k-1}, k \geq 2$. Se $p_{i}=\left(P_{1}, P_{2}, P_{3}\right)$ está em $B_{i}^{r}$, então $\Sigma_{i}^{r}\left(p_{i}\right)=\left(P_{1}-r a_{1}\left(p_{i}\right), P_{2}-\right.$ $\left.r a_{2}\left(p_{i}\right), P_{3}-r a_{3}\left(p_{i}\right)\right)$ esta em $B_{i}^{r}$.

Para calcular $D \Sigma_{i}^{r}(X)$, tomemos uma curva $\sigma(t)=\left(b_{1}(t), b_{2}(t), b_{3}(t)\right)$ com $X=$ $\left(b_{1}^{\prime}(0), b_{2}^{\prime}(0), b_{3}^{\prime}(0)\right)$, e calculemos a tangente para $\Sigma_{i}^{r}$ o $\sigma$ em $t=0$. Seja $N_{i}(\sigma(t))=$ $\left(a_{1}(t), a_{2}(t), a_{3}(t)\right)$; então $\Sigma_{i}^{r}$ o $\sigma=\left(b_{1}-r a_{1}(t), b_{2}-r a_{2}(t), b_{3}-r a_{3}(t)\right)$, e seu tangente em $t=0$ é dado por $X+r D N_{i}(X)$. Além disso, $N_{i}(\sigma(t))=N_{i}\left(\Sigma_{i}^{r} \circ \sigma(t)\right)=N_{i}^{r}\left(\Sigma_{i}^{r}\right.$ o $\sigma(t))$ da definição de $\sum_{i}^{r}$, de $B_{i}^{r}$ e que estamos trabalhando com normais unitárias. Desta forma $D N_{i}(X)=\left(a_{1}^{\prime}(0), a_{2}^{\prime}(0), a_{3}^{\prime}(0)\right)=D N_{i}\left(D \Sigma_{i}^{r}(X)\right)=D N_{i}^{r}\left(D \Sigma_{i}^{r}(X)\right)$.

Agora seja $E_{j}^{i}$ um vetor unitário em $p_{i} \in B_{i} \operatorname{com} D N_{i}\left(E_{j}^{i}\right)=k_{j}^{i} E_{j}^{i}$, então $D N_{i}\left(D \Sigma_{i}^{r}\left(E_{j}^{i}\right)\right)=D N_{i}\left(E_{j}^{i}\right)=k_{j}^{i} E_{j}^{i}$ e $D \Sigma_{i}^{r}\left(E_{j}^{i}\right)=\left(1-r k_{j}^{i}\right) E_{j}^{i}$. Se $1-r k_{j}^{i}=0$, então $D \Sigma_{i}^{r}\left(E_{j}^{i}\right)=0$ e $D N_{i}\left(D \Sigma_{i}^{r}\left(E_{j}^{i}\right)\right)=k_{j}^{i} E_{j}^{i}=0$, daí $k_{j}^{i}=0$ e $1=0$, portanto $1-r k_{j}^{i} \neq 0$ já que $B_{i}^{r}$ é uma superfície regular. Concluímos que $D N_{i}\left(D \Sigma_{i}^{r}\left(E_{j}^{i}\right)\right)=$ $\left(\frac{k_{j}^{i}}{1-r k_{j}^{i}}\right) D \Sigma_{i}^{r}\left(E_{j}^{i}\right)$, com isso mostramos que $\Sigma_{i}^{r}$ preserva as direções principais, umbílicos e que $k_{j}^{i}(r)=\frac{k_{j}^{i}}{1-r k_{j}^{i}}$.

As expressões de $\mathcal{K}^{i}(r)$ e $\mathcal{H}^{i}(r)$ saem dos seguintes cálculos:

$$
\begin{aligned}
\mathcal{K}^{i}(r) & =k_{1}^{i}(r) k_{2}^{i}(r)=\frac{k_{1}^{i} k_{2}^{i}}{\left(1-r k_{1}^{i}\right)\left(1-r k_{2}^{i}\right)}=\frac{\mathcal{K}^{i}}{1-2 r \mathcal{H}^{i}+r^{2} \mathcal{K}^{i}}, \\
2 \mathcal{H}^{i} & =k_{1}^{i}(r)+k_{2}^{i}(r)=\frac{k_{1}^{i}}{1-r k_{1}^{i}}+\frac{k_{2}^{i}}{1-r k_{2}^{i}}=\frac{k_{1}^{i}\left(1-r k_{2}^{i}\right)+k_{2}^{i}\left(1-r k_{1}^{i}\right)}{\left(1-r k_{1}^{i}\right)\left(1-r k_{2}^{i}\right)} \\
& =\frac{k_{1}^{i}+k_{2}^{i}-2 r k_{1}^{i} k_{2}^{i}}{\left(1-r k_{1}^{i}\right)\left(1-r k_{2}^{i}\right)}=\frac{2\left(\mathcal{H}^{i}-r \mathcal{K}^{i}\right)}{1-2 r \mathcal{H}^{i}+r^{2} \mathcal{K}^{i}} .
\end{aligned}
$$

O Lema acima pode ser estendido facilmente para hipersuperfícies no $\mathbb{R}^{n}$. A demonstração pode ser encontrada em (Hicks [6], p. 36 seção 2.6 ).

As expressões para $\tau_{g}^{i}, k_{n}^{i}$ e $k_{n \perp}^{i}$ em $B_{i}$, são definidas em termos das curvaturas principais, podendo ser obviamente modificadas para valerem em $B_{i}^{r}$ e denotaremos respectivamente por $\tau_{g}^{i}(r), k_{n}^{i}(r)$ e $k_{n \perp}^{i}(r)$. Portanto:

$$
\begin{gathered}
\tau_{g}^{i}(r)=\tau_{g}^{i}\left(r, \alpha_{i}\right)=\left(k_{2}^{i}(r)-k_{1}^{i}(r)\right) \operatorname{sen}\left(\alpha_{i}\right) \cos \left(\alpha_{i}\right), \\
k_{n}^{i}(r)=k_{n}^{i}\left(r, \alpha_{i}\right)=k_{1}^{i}(r) \cos ^{2}\left(\alpha_{i}\right)+k_{2}^{i}(r) \operatorname{sen}^{2}\left(\alpha_{i}\right), \\
k_{n \perp}^{i}(r)=k_{n \perp}^{i}\left(r, \alpha_{i}\right)=k_{1}^{i}(r) \operatorname{sen}^{2}\left(\alpha_{i}\right)+k_{2}^{i}(r) \cos ^{2}\left(\alpha_{i}\right) .
\end{gathered}
$$


Proposição 1 Com a notação acima, nos pontos de $C \backslash M$, temos

$$
\begin{aligned}
& D N . T_{1}=\frac{\operatorname{sen} \phi}{2}\left[k_{n}^{2}(r)-k_{n}^{1}(r)\right] T_{1}+\frac{1}{2}\left[\tau_{g}^{2}(r)-\tau_{g}^{1}(r)\right] T_{2}, \\
& D N . T_{2}=\frac{1}{2}\left[\tau_{g}^{2}(r)-\tau_{g}^{1}(r)\right] T_{1}+\frac{1}{2 \operatorname{sen} \phi}\left[k_{n \perp}^{2}(r)-k_{n \perp}^{1}(r)\right] T_{2} .
\end{aligned}
$$

Prova. A conclusão segue do cálculo dos produtos internos em

$$
\begin{aligned}
& D N\left(T_{1}\right)=\left\langle D N\left(T_{1}\right), T_{1}\right\rangle T_{1}+\left\langle D N\left(T_{1}\right), T_{2}\right\rangle T_{2} \\
& D N\left(T_{2}\right)=\left\langle D N\left(T_{2}\right), T_{1}\right\rangle T_{1}+\left\langle D N\left(T_{2}\right), T_{2}\right\rangle T_{2} .
\end{aligned}
$$

Diferenciando $N$, obtemos:

$$
D N=\frac{D N_{1} \cdot D \Pi_{1}^{r}-D N_{2} \cdot D \Pi_{2}^{r}}{2 \operatorname{sen} \phi}+\frac{1}{2} D\left[(\operatorname{sen} \phi)^{-1}\right] \cdot\left[N_{1}\left(\Pi_{1}(p)\right)-N_{2}\left(\Pi_{2}(p)\right)\right] .
$$

Como queremos fazer o produto interno de $D N$ por vetores tangentes, a contribuição do segundo termo é nula. Logo nos concentraremos somente no primeiro termo. Lembrando que,

$$
D \Pi_{i}^{r}\left(T_{1}\right)=\operatorname{sen} \phi F_{1}^{i} \text { e } D \Pi_{i}^{r}\left(T_{2}\right)=F_{2}^{i},
$$

e que as bases $E_{i}$ e $F_{i}$ são relacionadas por

$$
F_{1}^{i}=\cos \left(\alpha_{i}\right) E_{1}^{i}+\operatorname{sen}\left(\alpha_{i}\right) E_{2}^{i}, \quad F_{2}^{i}=-\operatorname{sen}\left(\alpha_{i}\right) E_{1}^{i}+\cos \left(\alpha_{i}\right) E_{2}^{i} .
$$

Calculemos agora $D N_{i} D \Pi_{i}^{r}$ nas direções $T_{1}$ e $T_{2}$, para sabermos o valor de $D N$ nestas direções.

$$
\begin{aligned}
D N_{i} \cdot D \Pi_{i}^{r}\left(T_{1}\right)= & D N_{i}\left(\operatorname{sen} \phi F_{1}^{i}\right) \\
= & \operatorname{sen} \phi D N_{i}\left(\cos \left(\alpha_{i}\right) E_{1}^{i}+\operatorname{sen}\left(\alpha_{i}\right) E_{2}^{i}\right) \\
= & \operatorname{sen} \phi\left[-k_{1}^{i}(r) \cos \left(\alpha_{i}\right) E_{1}^{i}-k_{2}^{i}(r) \operatorname{sen}\left(\alpha_{i}\right) E_{2}^{i}\right] \\
= & \operatorname{sen} \phi\left\{-k_{1}^{i}(r) \cos \left(\alpha_{i}\right)\left[\cos \left(\alpha_{i}\right) F_{1}^{i}-\operatorname{sen}\left(\alpha_{i}\right) F_{2}^{i}\right]\right. \\
& \left.-k_{2}^{i}(r) \operatorname{sen}\left(\alpha_{i}\right)\left[\operatorname{sen}\left(\alpha_{i}\right) F_{1}^{i}+\cos \left(\alpha_{i}\right) F_{2}^{i}\right]\right\} \\
= & \operatorname{sen} \phi\left\{\left[-k_{1}^{i}(r) \cos ^{2}\left(\alpha_{i}\right)-k_{2}^{i}(r) \operatorname{sen}^{2}\left(\alpha_{i}\right)\right] F_{1}^{i}\right. \\
& \left.+\left[k_{1}^{i}(r)-k_{2}^{i}(r)\right] \operatorname{sen}\left(\alpha_{i}\right) \cos \left(\alpha_{i}\right) F_{2}^{i}\right\} \\
= & \operatorname{sen} \phi\left\{\left[-k_{n}^{i}(r)\right] F_{1}^{i}+\left[-\tau_{g}^{i}(r)\right] F_{2}^{i}\right\} .
\end{aligned}
$$


Analogamente

$$
\begin{aligned}
D N_{i} . D \Pi_{i}^{r}\left(T_{2}\right)= & D N_{i}\left(F_{2}^{i}\right) \\
= & D N_{i}\left(-\operatorname{sen}\left(\alpha_{i}\right) E_{1}^{i}+\cos \left(\alpha_{i}\right) E_{2}^{i}\right) \\
= & {\left[k_{1}^{i}(r) \operatorname{sen}\left(\alpha_{i}\right) E_{1}^{i}-k_{2}^{i}(r) \cos \left(\alpha_{i}\right) E_{2}^{i}\right] } \\
= & \left\{k_{1}^{i}(r) \operatorname{sen}\left(\alpha_{i}\right)\left[\cos \left(\alpha_{i}\right) F_{1}^{i}-\operatorname{sen}\left(\alpha_{i}\right) F_{2}^{i}\right]\right. \\
& \left.-k_{2}^{i}(r) \cos \left(\alpha_{i}\right)\left[\operatorname{sen}\left(\alpha_{i}\right) F_{1}^{i}+\cos \left(\alpha_{i}\right) F_{2}^{i}\right]\right\} \\
= & \left\{\left[\left(k_{1}^{i}(r)-k_{2}^{i}(r)\right)\right] \operatorname{sen}\left(\alpha_{i}\right) \cos \left(\alpha_{i}\right) F_{1}^{i}\right. \\
& \left.-\left[k_{1}^{i}(r) \operatorname{sen}^{2}\left(\alpha_{i}\right)+k_{2}^{i}(r) \cos ^{2}\left(\alpha_{i}\right)\right] F_{2}^{i}\right\} \\
= & \left\{\left[-\tau_{g}^{i}(r)\right] F_{1}^{i}-\left[k_{n \perp}^{i}(r)\right] F_{2}^{i}\right\} .
\end{aligned}
$$

De (1.5), temos que

$$
\begin{aligned}
D N\left(T_{1}\right) & =\frac{\operatorname{sen} \phi}{2 \operatorname{sen} \phi}\left\{\left[-k_{n}^{1}(r) F_{1}^{1}-\tau_{g}^{1}(r) F_{2}^{1}\right]-\left[-k_{n}^{2}(r) F_{1}^{2}-\tau_{g}^{2}(r) F_{2}^{2}\right]\right\} \\
& =\frac{1}{2}\left\{\left[k_{n}^{2}(r) F_{1}^{2}-k_{n}^{1}(r) F_{1}^{1}\right]+\left[\tau_{g}^{2}(r) F_{2}^{2}-\tau_{g}^{1}(r) F_{2}^{1}\right]\right\} \\
D N\left(T_{2}\right) & =\frac{1}{2 \operatorname{sen} \phi}\left\{\left[\tau_{g}^{1} F_{1}^{1}-k_{n \perp}^{1}(r) F_{2}^{1}\right]-\left[-\tau_{g}^{2}(r) F_{1}^{2}-k_{n \perp}^{2}(r) F_{2}^{2}\right]\right\} \\
& =\frac{1}{2 \operatorname{sen} \phi}\left\{\left[k_{n \perp}^{2}(r) F_{2}^{2}-k_{n \perp}^{1}(r) F_{2}^{1}\right]+\left[\tau_{g}^{2}(r) F_{1}^{2}-\tau_{g}^{1}(r) F_{1}^{1}\right]\right\}
\end{aligned}
$$

Concluindo a demonstração, utilizamos os Lemas de 1 a 3 , para fazermos os produtos internos de (1.3) e (1.4), e chegamos as seguintes expressões:

$$
\begin{aligned}
\left\langle D N\left(T_{1}\right), T_{1}\right\rangle & =\frac{\operatorname{sen} \phi}{2}\left[k_{n}^{2}(r)-k_{n}^{1}(r)\right], \\
\left\langle D N\left(T_{1}\right), T_{2}\right\rangle & =\frac{1}{2}\left[\tau_{g}^{2}(r)-\tau_{g}^{1}(r)\right], \\
\left\langle D N\left(T_{2}\right), T_{1}\right\rangle & =\frac{1}{2}\left[\tau_{g}^{2}(r)-\tau_{g}^{1}(r)\right], \\
\left\langle D N\left(T_{2}\right), T_{2}\right\rangle & =\frac{1}{2 \operatorname{sen} \phi}\left[k_{n \perp}^{2}(r)-k_{n \perp}^{1}(r)\right] .
\end{aligned}
$$

Note que a prova da proposição usa somente a base principal no ponto (e não em conjuntos abertos) de $B_{i}$. Além disso, os cálculos de $D N$ são válidos também nos pontos de $C \backslash M$, cuja projeção em uma (ou ambas) as superfícies $B_{i}$ são pontos umbílicos. Neste caso, temos $k_{1}^{i}(r)=k_{2}^{i}(r)$ e podemos considerar $\alpha_{i}=0$ (já que 
toda direção é principal), assim concluímos que valem as seguintes igualdades para. as projeções umbílicas,

$$
\tau_{g}^{i}(r)=0 \quad \text { e } \quad k_{n}^{i}(r)=k_{1}^{i}(r)=k_{n \perp}^{i}(r)=k_{2}^{i}(r)=k^{i}(r)
$$

logo no caso das duas projeções serem umbílicas, obtemos pela proposição anterior

$$
\begin{aligned}
& D N\left(T_{1}\right)=\frac{\operatorname{sen} \phi}{2}\left[k^{2}(r)-k^{1}(r)\right] T_{1}, \\
& D N\left(T_{2}\right)=\frac{1}{2 \operatorname{sen} \phi}\left[k^{2}(r)-k^{1}(r)\right] T_{2} .
\end{aligned}
$$

Teorema 1 Sejam $\mathcal{K}^{i}=\mathcal{K}^{i}(r)=k_{1}^{i}(r) k_{2}^{i}(r), \mathcal{H}^{i}=\mathcal{H}^{i}(r)=\frac{1}{2}\left(k_{1}^{i}(r)+k_{2}^{i}(r)\right), \mathcal{U}^{i}=$ $\mathcal{U}^{i}(r)=\frac{1}{2}\left(k_{2}^{i}(r)-k_{1}^{i}(r)\right)$, onde $k_{j}^{i}(r)=\frac{k_{j}^{i}}{1-r k_{j}^{i}}$.

a) A curvatura Gaussiana da superfície de conflito $C$ é dada por

$$
\mathcal{K}=\left[\frac{1}{2}\left(\mathcal{K}^{1}+\mathcal{K}^{2}\right)-\mathcal{H}^{1} \mathcal{H}^{2}+\cos \left(2\left(\alpha_{2}-\alpha_{1}\right)\right) \mathcal{U}^{1} \mathcal{U}^{2}\right]
$$

b) A curvatura média da superfície de conflito $C$ é dada por

$$
\mathcal{H}=\left(\frac{1+\operatorname{sen}^{2} \phi}{4 \operatorname{sen} \phi}\right)\left[\left(\mathcal{H}^{1}-\mathcal{H}^{2}\right)+\left(\frac{\cos ^{2} \phi}{1+\operatorname{sen} \phi}\right)\left(\cos \left(2 \alpha_{1}\right) \mathcal{U}^{1}-\cos \left(2 \alpha_{2}\right) \mathcal{U}^{2}\right)\right]
$$

Prova. a) A curvatura Gaussiana de $C$ é dada por $\operatorname{det}(-D N)$. Além disso, precisaremos no decorrer dos cálculos que

$$
\begin{aligned}
k_{n}^{i}(r)= & k_{1}^{i}(r) \cos ^{2}\left(\alpha_{i}\right)+k_{2}^{i}(r) \operatorname{sen}^{2}\left(\alpha_{i}\right) \\
= & \frac{1}{2} k_{1}^{i}(r)\left[1-\operatorname{sen}^{2}\left(\alpha_{i}\right)\right]+\frac{1}{2} k_{1}^{i}(r) \cos ^{2}\left(\alpha_{i}\right)+\frac{1}{2} k_{2}^{i}(r)\left[1-\cos ^{2}\left(\alpha_{i}\right)\right]+\frac{1}{2} k_{2}^{i}(r) \operatorname{sen}^{2}\left(\alpha_{i}\right) \\
= & \frac{1}{2}\left[k_{1}^{i}(r)+k_{2}^{i}(r)\right]-\frac{1}{2}\left[k_{2}^{i}(r) \cos ^{2}\left(\alpha_{i}\right)-k_{1}^{i}(r) \cos ^{2}\left(\alpha_{i}\right)-k_{2}^{i}(r) \operatorname{sen}^{2}\left(\alpha_{i}\right)\right. \\
& \left.\quad+k_{1}^{i}(r) \operatorname{sen}^{2}\left(\alpha_{i}\right)\right] \\
= & \mathcal{H}^{i}-\mathcal{U}^{i} \cos \left(2 \alpha_{i}\right), \\
k_{n \perp}^{i}(r)= & k_{1}^{i}(r) \operatorname{sen}^{2}\left(\alpha_{i}\right)+k_{2}^{i}(r) \cos ^{2}\left(\alpha_{i}\right) \\
= & \frac{1}{2} k_{1}^{i}(r)\left[1-\cos ^{2}\left(\alpha_{i}\right)\right]+\frac{1}{2} k_{1}^{i}(r) \operatorname{sen}^{2}\left(\alpha_{i}\right)+\frac{1}{2} k_{2}^{i}(r)\left[1-\operatorname{sen}^{2}\left(\alpha_{i}\right)\right]+\frac{1}{2} k_{2}^{i}(r) \cos ^{2}\left(\alpha_{i}\right) \\
= & \frac{1}{2}\left[k_{1}^{i}(r)+k_{2}^{i}(r)\right]+\frac{1}{2}\left[k_{2}^{i}(r) \cos ^{2}\left(\alpha_{i}\right)-k_{1}^{i}(r) \cos ^{2}\left(\alpha_{i}\right)-k_{2}^{i}(r) \operatorname{sen}^{2}\left(\alpha_{i}\right)\right. \\
\left.\quad \quad+k_{1}^{i}(r) \operatorname{sen}^{2}\left(\alpha_{i}\right)\right] &
\end{aligned}
$$




$$
\begin{aligned}
\tau_{g}^{2}(r)-\tau_{g}^{1}(r) & =\left[k_{2}^{2}(r)-k_{1}^{2}(r)\right] \operatorname{sen}\left(\alpha_{2}\right) \cos \left(\alpha_{2}\right)-\left[k_{2}^{1}(r)-k_{1}^{1}(r)\right] \operatorname{sen}\left(\alpha_{1}\right) \cos \left(\alpha_{1}\right) \\
& =2 \mathcal{U}^{2} \operatorname{sen}\left(\alpha_{2}\right) \cos \left(\alpha_{2}\right)-2 \mathcal{U}^{1} \operatorname{sen}\left(\alpha_{1}\right) \cos \left(\alpha_{1}\right) \\
& =\mathcal{U}^{2} \operatorname{sen}\left(2 \alpha_{2}\right)-\mathcal{U}^{1} \operatorname{sen}\left(2 \alpha_{1}\right), \\
\left(\mathcal{U}^{i}\right)^{2} & =\left(\mathcal{H}^{i}\right)^{2}-\mathcal{K}^{i} .
\end{aligned}
$$

obtemos

$$
\begin{aligned}
\mathcal{K}= & \frac{1}{4}\left\{\left[k_{n}^{2}(r)-k_{n}^{1}(r)\right]\left[k_{n \perp}^{2}(r)-k_{n \perp}^{1}(r)\right]-\left[\tau_{g}^{2}(r)-\tau_{g}^{1}(r)\right]^{2}\right\} \\
= & \frac{1}{4}\left\{[ ( \mathcal { H } ^ { 2 } - \mathcal { U } ^ { 2 } \operatorname { c o s } ( 2 \alpha _ { 2 } ) ) - ( \mathcal { H } ^ { 1 } - \mathcal { U } ^ { 1 } \operatorname { c o s } ( 2 \alpha _ { 1 } ) ) ] \left[\left(\mathcal{H}^{2}+\mathcal{U}^{2} \cos \left(2 \alpha_{2}\right)\right.\right.\right. \\
& \left.\left.\left.\quad-\mathcal{H}^{1}+\mathcal{U}^{1} \cos \left(2 \alpha_{1}\right)\right)\right]-\left[\mathcal{U}^{2} \operatorname{sen}\left(2 \alpha_{2}\right)-\mathcal{U}^{1} \operatorname{sen}\left(2 \alpha_{1}\right)\right]^{2}\right\} \\
= & \frac{1}{4}\left\{\left(\mathcal{H}^{2}\right)^{2}-\left(\mathcal{U}^{2} \cos \left(2 \alpha_{2}\right)\right)^{2}+\left(\mathcal{H}^{1}\right)^{2}-\left(\mathcal{U}^{1} \cos \left(2 \alpha_{1}\right)\right)^{2}-2 \mathcal{H}^{1} \mathcal{H}^{2}\right. \\
& \left.+2 \mathcal{U}^{1} \mathcal{U}^{2} \cos \left(2 \alpha_{1}\right) \cos \left(2 \alpha_{2}\right)-\left[\mathcal{U}^{2} \operatorname{sen}\left(2 \alpha_{2}\right)-\mathcal{U}^{1} \operatorname{sen}\left(2 \alpha_{1}\right)\right]^{2}\right\} \\
= & \frac{1}{4}\left\{\left(\mathcal{H}^{2}\right)^{2}-\left(\mathcal{U}^{2}\right)^{2}+\left(\mathcal{U}^{2} \operatorname{sen}\left(2 \alpha_{2}\right)\right)^{2}+\left(\mathcal{H}^{1}\right)^{2}-\left(\mathcal{U}^{1}\right)^{2}+\left(\mathcal{U}^{1} \operatorname{sen}\left(2 \alpha_{1}\right)\right)^{2}-2 \mathcal{H}^{1} \mathcal{H}^{2}\right. \\
& \left.\quad+2 \mathcal{U}^{1} \mathcal{U}^{2} \cos \left(2 \alpha_{1}\right) \cos \left(2 \alpha_{2}\right)-\left[\mathcal{U}^{2} \operatorname{sen}\left(2 \alpha_{2}\right)-\mathcal{U}^{1} \operatorname{sen}\left(2 \alpha_{1}\right)\right]^{2}\right\} \\
= & \frac{1}{4}\left\{\left(\mathcal{K}^{1}+\mathcal{K}^{2}\right)-2 \mathcal{H}^{1} \mathcal{H}^{2}+2 \mathcal{U}^{1} \mathcal{U}^{2} \cos \left(2\left(\alpha_{1}-\alpha_{2}\right)\right)\right\}
\end{aligned}
$$

b) A curvatura média é dada pelo traço(- $\left.\frac{1}{2} D N\right)$. Deduzimos usando as expressões da parte $a)$ que

$$
\begin{aligned}
\mathcal{H}= & -\frac{1}{4}\left\{\operatorname{sen} \phi\left[k_{n}^{2}(r)-k_{n}^{1}(r)\right]+\frac{1}{\operatorname{sen} \phi}\left[k_{n \perp}^{2}(r)-k_{n \perp}^{1}(r)\right]\right\} \\
= & -\frac{1}{4}\left\{\operatorname{sen} \phi\left[\mathcal{H}^{2}-\mathcal{H}^{1}+\mathcal{U}^{1} \cos \left(2 \alpha_{1}\right)-\mathcal{U}^{2} \cos \left(2 \alpha_{2}\right)\right]+\frac{1}{\operatorname{sen} \phi}\left[\mathcal{H}^{2}-\mathcal{H}^{1}\right.\right. \\
& \left.+\mathcal{U}^{2} \cos \left(2 \alpha_{2}\right)-\mathcal{U}^{1} \cos \left(2 \alpha_{1}\right)\right] \\
= & -\frac{1}{4}\left\{\left[\mathcal{H}^{2}-\mathcal{H}^{1}\right]\left(\operatorname{sen} \phi+\frac{1}{\operatorname{sen} \phi}\right)+\left[\mathcal{U}^{1} \cos \left(2 \alpha_{1}\right)-\mathcal{U}^{2} \cos \left(2 \alpha_{2}\right)\right]\left(\operatorname{sen} \phi-\frac{1}{\operatorname{sen} \phi}\right)\right\} \\
= & \left(\frac{1+\operatorname{sen}^{2} \phi}{4 \operatorname{sen} \phi}\right)\left\{\left[\mathcal{H}^{1}-\mathcal{H}^{2}\right]+\left(\frac{\cos ^{2} \phi}{1+\operatorname{sen}^{2} \phi}\right)\left[\mathcal{U}^{1} \cos \left(2 \alpha_{1}\right)-\mathcal{U}^{2} \cos \left(2 \alpha_{2}\right)\right]\right\}
\end{aligned}
$$

Corolário 1 ponto $p \in C \backslash M$ é umbílico se somente se $\Upsilon=\Psi=0$, onde

$$
\begin{aligned}
\Upsilon & =\left[\tau_{g}^{2}(r)-\tau_{g}^{1}(r)\right]=\mathcal{U}^{2} \operatorname{sen}\left(2 \alpha_{2}\right)-\mathcal{U}^{1} \operatorname{sen}\left(2 \alpha_{1}\right), \\
\Psi & =\operatorname{sen}^{2} \phi\left[k_{n}^{2}(r)-k_{n}^{1}(r)\right]-\left[k_{n \perp}^{2}(r)-k_{n \perp}^{1}(r)\right] \\
& =\left[\mathcal{H}^{1}-\mathcal{H}^{2}\right] \cos ^{2} \phi+\left(1+\operatorname{sen}^{2} \phi\right)\left[\mathcal{U}^{1} \cos \left(2 \alpha_{1}\right)-\mathcal{U}^{2} \cos \left(2 \alpha_{2}\right)\right] \\
& =\left(\frac{3-\cos 2 \phi}{2}\right)\left\{\left[\mathcal{H}^{1}-\mathcal{H}^{2}\right]\left(\frac{1+\cos 2 \phi}{3-\cos 2 \phi}\right)+\left[\mathcal{U}^{1} \cos \left(2 \alpha_{1}\right)-\mathcal{U}^{2} \cos \left(2 \alpha_{2}\right)\right]\right\} .
\end{aligned}
$$


Prova. $(\Rightarrow)$ Se $p$ é um ponto umbílico temos que toda direção tangente em $p$ é principal. Disto e das expressões (1.1) e (1.2), temos

$$
\begin{aligned}
& D N . T_{1}=\tilde{k} T_{1}=\frac{\operatorname{sen} \phi}{2}\left[k_{n}^{2}(r)-k_{n}^{1}(r)\right] T_{1} \\
& D N . T_{2}=\hat{k} T_{2}=\frac{1}{2 \operatorname{sen} \phi}\left[k_{n \perp}^{2}(r)-k_{n \perp}^{1}(r)\right] T_{2}
\end{aligned}
$$

A contribuição de $T_{2}$ em (1.6) é nula. Logo de (1.1), temos

$$
\Upsilon=\left(\tau_{g}^{2}(r)-\tau_{g}^{1}(r)\right)=0
$$

analogamente, poderíamos obter a mesma expressão levando em conta que a contribuição de $T_{1}$ é nula em (1.7), podemos recorrer a expressão (1.2) para obtermos o mesmo resultado.

Com isto a matriz de $D N$ esta diagonalizada, ou seja, seus elementos da diagonal são os autovalores de $D N$. Como $p$ é umbílico, temos

$$
\begin{aligned}
\tilde{k}=\hat{k} & \Rightarrow \tilde{k}-\hat{k}=0 \\
& \Rightarrow \frac{\operatorname{sen} \phi}{2}\left[k_{n}^{2}(r)-k_{n}^{1}(r)\right]-\frac{1}{2 \operatorname{sen} \phi}\left[k_{n \perp}^{2}(r)-k_{n \perp}^{1}(r)\right]=0 \\
& \Rightarrow \operatorname{sen}^{2} \phi\left[k_{n}^{2}(r)-k_{n}^{1}(r)\right]-\left[k_{n \perp}^{2}(r)-k_{n \perp}^{1}(r)\right]=0 \\
& \Rightarrow \Psi=0
\end{aligned}
$$

$(\Leftarrow)$ Se $\Upsilon=0$ então a matriz de $D N$ fica da seguinte forma

$$
D N=\left(\begin{array}{cc}
\frac{\operatorname{sen} \phi}{2}\left(k_{n}^{2}(r)-k_{n}^{1}(r)\right) & 0 \\
0 & \frac{1}{2 \operatorname{sen} \phi}\left(k_{n \perp}^{2}(r)-k_{n \perp}^{1}(r)\right)
\end{array}\right)
$$

Como $\Psi=0$, a subtração dos elementos da diagonal é zero. Daí, concluímos que a subtração dos autovalores de $D N$ é zero, ou seja, $k_{1}-k_{2}=0 \Rightarrow k_{1}=k_{2} \Rightarrow p$ é umbílico

Corolário 2 As direções principais em um ponto $p$ não umbílico de $C \backslash M$ são caracterizadas por fazerem um ângulo $\theta$ com $T_{1}$, dado por

$$
\begin{aligned}
\tan 2 \theta & =\frac{2\left[\tau_{g}^{2}(r)-\tau_{g}^{1}(r)\right] \operatorname{sen} \theta}{\operatorname{sen}^{2} \phi\left[k_{n}^{2}(r)-k_{n}^{1}(r)\right]-\left[k_{n \perp}^{2}(r)-k_{n \perp}^{1}(r)\right]} \\
\tan 2 \theta & =\frac{2 \Upsilon \operatorname{sen} \theta}{\Psi}
\end{aligned}
$$


Prova. Seja X um vetor unitário do plano tangente no ponto $p \in C \backslash M$. Escrevendo $X$ na base $\left[T_{1}, T_{2}\right]$ e chamando de $\bar{X}$ o vetor ortogonal a $X$, neste plano, temos que a. torção geodésica na direção $X$ é dada por

$$
\begin{aligned}
\tau_{g}(X) & =-\langle D N X, \bar{X}\rangle \\
\tau_{g}(X) & =-\left\langle D N\left(\cos \theta T_{1}+\operatorname{sen} \theta T_{2}\right),-\operatorname{sen} \theta T_{1}+\cos \theta T_{2}\right\rangle \\
\tau_{g}(X) & =\left(\operatorname{sen}^{2} \theta-\cos ^{2} \theta\right)\left\langle D N T_{1}, T_{2}\right\rangle+\cos \theta \operatorname{sen} \theta\left(\left\langle D N T_{1}, T_{1}\right\rangle-\left\langle D N T_{2}, T_{2}\right\rangle\right)
\end{aligned}
$$

Daí, X é uma direção principal, se somente se, $\tau_{g}(X)=0$. Portanto igualando a zero (1.5), obtemos

$$
\begin{aligned}
0=\left(\operatorname{sen}^{2} \theta\right. & \left.-\cos ^{2} \theta\right)\left\langle D N T_{1}, T_{2}\right\rangle+\cos \theta \operatorname{sen} \theta\left(\left\langle D N T_{1}, T_{1}\right\rangle-\left\langle D N T_{2}, T_{2}\right\rangle\right) \\
\frac{2 \cos \theta \operatorname{sen} \theta}{\left(\cos ^{2} \theta-\operatorname{sen}^{2} \theta\right)} & =\frac{2\left\langle D N T_{1}, T 2\right\rangle}{\left(\left\langle D N T_{1}, T_{1}\right\rangle-\left\langle D N T_{2}, T_{2}\right\rangle\right)} \\
\tan 2 \theta & =\frac{2\left(\frac{1}{2}\left[\tau_{g}^{2}(r)-\tau_{g}^{1}(r)\right]\right)}{\frac{\operatorname{sen} \phi}{2}\left[k_{n}^{2}(r)-k_{n}^{1}(r)\right]-\frac{1}{\operatorname{sen} \phi}\left[k_{n \perp}^{2}(r)-k_{n \perp}^{1}(r)\right]} \\
\tan 2 \theta & =\frac{2\left[\tau_{g}^{2}(r)-\tau_{g}^{1}(r)\right] \operatorname{sen} \phi}{\operatorname{sen}^{2} \phi\left[k_{n}^{2}(r)-k_{n}^{1}(r)\right]-\left[k_{n \perp}^{2}(r)-k_{n \perp}^{1}(r)\right]} \\
\tan 2 \theta & =\frac{2 \Upsilon \operatorname{sen} \phi}{\Psi}
\end{aligned}
$$

Corolário 3 Seja $X$ um vetor unitário tangente a $C / M$ em p. Então a curvatura normal na direção de $X$ é dada por

$$
\begin{aligned}
k_{n}(X)=-\left\{\frac{\cos ^{2}(\theta) \operatorname{sen} \phi}{2}\left[k_{n}^{2}(r)-k_{n}^{1}(r)\right]+\right. & \frac{\operatorname{sen}^{2}(\theta)}{2 \operatorname{sen} \phi}\left[k_{n \perp}^{2}(r)-k_{n \perp}^{1}(r)\right] \\
& \left.+\cos (\theta) \operatorname{sen}(\theta)\left[\tau_{g}^{2}(r)-\tau_{g}^{1}(r)\right]\right\},
\end{aligned}
$$

onde $\theta$ é o ângulo entre $X$ e $T_{1}$.

Prova. Como $X$ um vetor unitário tangente a $C / M$ em $p, \mathrm{X}$ pode ser escrito da forma $X=\cos \theta T_{1}+\operatorname{sen} \theta T_{2}$. Daí temos que

$$
\begin{aligned}
& k_{n}(X)=-\langle D N(X), X\rangle \\
&=-\left\langle D N\left(\cos \theta T_{1}+\operatorname{sen} \theta T_{2}\right), \cos \theta T_{1}+\operatorname{sen} \theta T_{2}\right\rangle \\
&=-\cos ^{2} \theta\left\langle D N\left(T_{1}\right), T_{1}\right\rangle+\operatorname{sen}^{2} \theta\left\langle D N\left(T_{2}\right), T_{2}\right\rangle+2 \cos \theta \operatorname{sen} \theta\left\langle D N\left(T_{1}\right), T_{2}\right\rangle \\
&=-\left\{\frac{\cos ^{2} \theta \operatorname{sen} \phi}{2}\left[k_{n}^{2}(r)-k_{n}^{1}(r)\right]+\frac{\operatorname{sen}^{2} \theta}{2 \operatorname{sen} \phi}\left[k_{n \perp}^{2}(r)-k_{n \perp}^{1}(r)\right]\right. \\
&\left.\quad+\cos \theta \operatorname{sen} \theta\left[\tau_{g}^{2}(r)-\tau_{g}^{1}(r)\right]\right\} .
\end{aligned}
$$


Note, que igualando a zero a expressão acima para a curvatura normal obtemos uma expressão para as direções assintóticas em função do ângulo $\theta$ e dos dados relativos aos $B_{i}$, analogamente a que obtemos para as direções principais. A seguir, temos um corolário que nos dar a expressão de $k_{n}$ em um ponto de $M$.

Corolário 4 Seja $p=p_{m} \in M$ e suponha que $p_{i}=\Pi_{i}(p)$ não é ponto umbilico de $B_{i}, i=1,2$. Seja $X$ um vetor unitário de $T_{p} C$ e $\theta$ o ângulo formado entre $X$ e $E_{1}^{1}$. Então a curvatura normal na direção $X$ é dada por

$$
k_{n}(X)=k_{n}(\theta)=-\frac{1}{2}\left[k_{n}^{2}(r)-k_{n}^{1}(r)\right] .
$$

Prova. Seja $X$ um vetor unitário tangente a $C$ em $p$. Como $N_{1}$ e $N_{2}$ são paralelos neste ponto temos que $X$ pode ser escrito das seguintes formas:

$$
X=\cos \theta E_{1}^{1}+\operatorname{sen} \theta E_{2}^{1}=\cos (\theta+\beta) E_{1}^{2}+\operatorname{sen}(\theta+\beta) E_{2}^{2}
$$

onde $\theta$ é o ângulo que $X$ faz com $E_{1}^{1}$ e $\beta$ é o ângulo entre $E_{1}^{1}$ e $E_{1}^{2}$.

Calculemos então a curvatura normal na direção $X$, que é dada por

$$
k_{n}(X)=k_{n}(\theta)=-\langle D N(X), X\rangle \text {, }
$$

da expressão de $D N$, em (1.5), só é necessário a primeira primeira parcela, já que vamos fazer um produto interno com um vetor tangente $X$. Daí

$$
\begin{aligned}
= & -\left\langle\frac{D N_{1} D \Pi_{1}^{r}(X)-D N_{2} D \Pi_{2}^{r}(X)}{2 \operatorname{sen} \phi}, X\right\rangle \\
= & \frac{-1}{2 \operatorname{sen} \phi}\left[\left\langle D N_{1} D \Pi_{1}^{r}(X), X\right\rangle-\left\langle D N_{2} D \Pi_{2}^{r}(X), X\right\rangle\right] \\
= & \frac{-1}{2 \operatorname{sen} \phi}\left[\left\langle D N_{1} D \Pi_{1}^{r}\left(\cos (\theta) E_{1}^{1}+\operatorname{sen}(\theta) E_{2}^{1}\right), \cos (\theta) E_{1}^{1}+\operatorname{sen}(\theta) E_{2}^{1}\right\rangle\right. \\
& \left.-\left\langle D N_{2} D \Pi_{2}^{r}\left(\cos (\theta+\beta) E_{1}^{2}+\operatorname{sen}(\theta+\beta) E_{2}^{2}\right), \cos (\theta+\beta) E_{1}^{2}+\operatorname{sen}(\theta+\beta) E_{2}^{2}\right\rangle\right] \\
= & \frac{-1}{2 \operatorname{sen} \phi}\left[\left(-k_{1}^{1}(r) \cos ^{2}(\theta)-k_{2}^{1}(r) \operatorname{sen}^{2}(\theta)\right)-\left(-k_{1}^{2}(r) \cos ^{2}(\theta+\beta)-k_{2}^{2}(r) \operatorname{sen}^{2}(\theta+\beta)\right)\right] \\
= & \frac{-1}{2 \operatorname{sen} \phi}\left[k_{n}^{2}(r, \theta)-k_{n}^{1}(r, \theta)\right],
\end{aligned}
$$

como $\phi=\frac{\pi}{2}$, obtemos a expressão desejada.

Já que $k_{n}(X)$, a curvatura normal na direção do vetor unitário $X \in T_{p} C$, não depende da escolha da curva regular $\gamma$, tal que $\gamma(0)=p$ e $\gamma^{\prime}(0)=X$. Podemos 
utilizar da curva gerada pela intersecção do plano ortogonal a $X \wedge N(p) \operatorname{com} C$. Se a curvatura da curva no ponto $p$ é maior que zero, $k(0)>0$, então o vetor normal $n(0)= \pm N(p)$ e portanto, segue-se que

$$
k_{n}(X)= \pm k(0)
$$

disto podemos enunciar o seguinte teorema:

Teorema 2 Seja $\gamma$ dada como acima, na qual $\gamma^{\prime}(0)=T_{1}$. Suponhamos que $F_{1}^{i}$ são direçôes principais. Então a curvatura da curva $\gamma$ em $\gamma(0)=p$, é dada por

$$
k(0)=-\frac{\operatorname{sen} \phi}{2}\left[\frac{k_{j}^{2}}{1-r k_{j}^{2}}-\frac{k_{j}^{1}}{1-r k_{j}^{1}}\right]
$$

quando $F_{1}^{i}$ é paralelo a $E_{j}^{i}$.

Prova. Supondo o sinal positivo em (1.9) e utilizando o corolário 3, temos a seguinte expressão para a curvatura de $\gamma$ em $p$ onde $\gamma^{\prime}(0)=X$,

$$
\begin{aligned}
k(0)=-\left\{\frac{\cos ^{2}(\theta) \operatorname{sen} \phi}{2}\left[k_{n}^{2}(r)-k_{n}^{1}(r)\right]+\right. & \frac{\operatorname{sen}^{2}(\theta)}{2 \operatorname{sen} \phi}\left[k_{n \perp}^{2}(r)-k_{n \perp}^{1}(r)\right] \\
& \left.+\cos (\theta) \operatorname{sen}(\theta)\left[\tau_{g}^{2}(r)-\tau_{g}^{1}(r)\right]\right\} .
\end{aligned}
$$

Para o caso de $X=T_{1}$ e os vetores $F_{1}^{i}$ serem direções principais, basta fazer $\theta=0$ e $\alpha_{i}=0$. Daí obtemos

$$
k(0)=-\frac{\operatorname{sen} \phi}{2}\left[\frac{k_{j}^{2}}{1-r k_{j}^{2}}-\frac{k_{j}^{1}}{1-r k_{j}^{1}}\right]
$$

quando $F_{1}^{i}$ é paralelo a $E_{j}^{i}$.

Podemos ver facilmente que no caso do $\mathbb{R}^{2}$, a forma de procedimento para encontrarmos a curvatura da curva de conflito definida por dois conjuntos convexos $A_{1}$ e $A_{2}$ é semelhante. Já que $T_{1}=T$ e $N$ são definidos como a subtração e a soma das normais $N_{i}$, pelas propriedades análogas as citadas aqui, podemos mostrar que estes dois vetores são respectivamente tangente e normal as curvas de conflito do $\mathbb{R}^{2}$. Utilizando-se das mesmas definições para $F_{1}^{i}=F^{i}=\nu\left(D \Pi_{i}(p) \cdot T_{1}\right)$, teremos que estes vetores são tangentes a.os bordos $B_{i}$ dos conjuntos $A_{i}$.

Achamos bastante natural, fazermos estas associações, pois assim podemos chegar na expressão obtida por Siersma em [11]. Abaixo, mostraremos como obter a curvatura das curvas de conflito em função dos dados geométricos dos $B_{i}$ no $\mathbb{R}^{2}$. 
Teorema 3 Sejam $A_{1}$ e $A_{2}$ dois conjuntos convexos, disjuntos e com bordo $C^{k}, k \geq$ 2, no plano $\mathbb{R}^{2}$. Então

$$
k=-\frac{\operatorname{sen} \phi}{2}\left[\frac{k_{2}}{1-r k_{2}}-\frac{k_{1}}{1-r k_{1}}\right]
$$

onde $k$ é a curvatura em um ponto $p$ do conjunto de conflito, $k_{i}$ são as curvaturas nos pontos $p_{i}=\Pi_{i}(p)$ em $B_{i}$ e $r$ é a distância de $p$ para $A_{i}$.

Prova. Diferenciando $N$, obtemos:

$$
d N=\frac{d N_{1} \cdot d \Pi_{1}^{r}-d N_{2} \cdot d \Pi_{2}^{r}}{2 \operatorname{sen} \phi}+\frac{1}{2} d\left[(\operatorname{sen} \phi)^{-1}\right] \cdot\left[N_{1}\left(\Pi_{1}(p)\right)-N_{2}\left(\Pi_{2}(p)\right)\right] .
$$

Como queremos fazer o produto interno de $d N$ pelo vetor tangente $T$, a contribuição do segundo termo é nulo. Logo nos concentraremos somente no primeiro termo. Lembrando que,

$$
d \Pi_{i}^{r}(T)=\operatorname{sen} \phi F^{i}
$$

Calculemos agora $d N_{i} \cdot d \Pi_{i}^{r}$ na direção de $T$, para sabermos o valor de $d N$ nesta direção.

$$
d N_{i} \cdot d \Pi_{i}^{r}(T)=d N_{i}\left(\operatorname{sen} \phi F^{i}\right)=\operatorname{sen} \phi d N_{i}\left(F^{i}\right)=\operatorname{sen} \phi\left[-k^{i}(r) F^{i}\right]
$$

Daí chegamos à seguinte expressão para $d N$,

$$
d N=\frac{1}{2}\left[k^{2}(r) F^{2}-k^{1}(r) F^{1}\right]
$$

Para calcular a curvatura $k$ do conflito é suficiente fazer o produto interno

$$
\begin{aligned}
k & =\langle-d N, T\rangle=-\left\langle\frac{k^{2}(r) F^{2}-k^{1}(r) F^{1}}{2}, T\right\rangle \\
& =-\frac{\operatorname{sen} \phi}{2}\left[k^{2}(r)-k^{1}(r)\right]=-\frac{\operatorname{sen} \phi}{2}\left[\frac{k_{2}}{1-r k_{2}}-\frac{k_{1}}{1-r k_{1}}\right] .
\end{aligned}
$$

Lembramos, que a orientação dos vetores $N_{i}$ usada, neste trabalho é para o interior dos conjuntos $A_{i}$. No artigo de Siersma [11], a orientação escolhida para as normais $N_{i}$ é para o exterior dos conjuntos $A_{i}$. Por este motivo, houveram algumas mudanças de sinais. Na figura 1.4 temos um noção de como os vetores estão dispostos.

No seção seguinte estudaremos o comportamento das linhas de curvatura na vizinhança de um ponto umbílico. Faremos uma introdução do tipo umbílico que pode surgir em $p_{m} \in M$. 


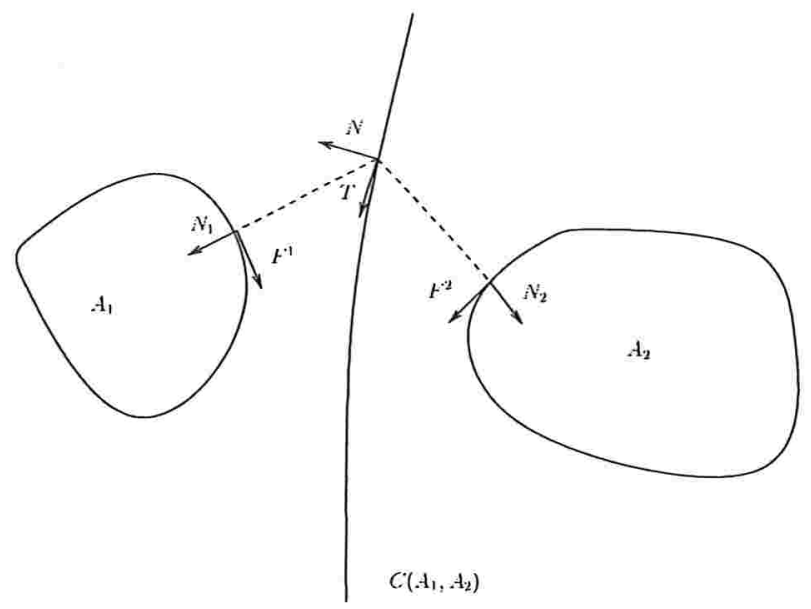

Figura 1.4: Conflito no Plano

\subsection{Pontos Umbílicos nas Superfícies de Conflito}

Nesta seção será mostrado como determinar o tipo Darbouxiano de um ponto umbílico $p_{m} \in M$.

Faremos uma pequena introdução sobre pontos umbílicos. Estabeleceremos condições para que um ponto umbílico seja Darbouxiano, assim como a configuração principal na vizinhança desse pontos.

Seja $p_{o} \in S$ um ponto umbílico da superfície $S$. Considere a carta $(u, v):\left(S, p_{o}\right) \rightarrow$ $\left(\mathbb{R}^{2}, 0\right)$ em torno de $p_{o}$, onde a superfície é o gráfico de uma função da forma:

$$
w=\frac{k}{2}\left(u^{2}+v^{2}\right)+\frac{a}{6} u^{3}+\frac{b}{2} u v^{2}+\frac{c}{6} v^{3}+O\left[\left(u^{2}+v^{2}\right)^{2}\right] .
$$

Isto é obtido através da projeção de $S$ em $T S\left(p_{o}\right)$ ao longo da normal $N\left(p_{o}\right)$ e escolhendo uma carta ortonormal $(u, v)$ em que o coeficiente de termo cúbico $u^{2} v$ da projeção, $w$, de $S$ em $N\left(p_{o}\right)$ é nulo.

Um ponto umbílico é chamado Darbouxiano se satisfaz, para a carta acima, as seguintes condições, $T$ e $D$, como segue:

T) A Condição de Transversalidade: $b(b-a) \neq 0$

D) A Condição Discriminante:

$\left.D_{1}\right):\left(\frac{c}{2 b}\right)^{2}-\frac{a}{b}+2<0$

$\left.D_{2}\right):\left(\frac{c}{2 b}\right)^{2}+2>\frac{a}{b}>1, a \neq b$

$\left.D_{3}\right): \frac{a}{b}<1$. 
Na carta $(u, v)$ acima, as equações para as linhas principais são escritas por

$$
[b v+L] d v^{2}-[(b-a) u+c v+M] d u d v-[b v+N] d u^{2}=0
$$

onde $L, M$ e $N$ são funções de ordem $O\left[\left(u^{2}+v^{2}\right)^{2}\right]$.

A condição $\mathbf{T}$, implica que as curvas

$$
b v+L=0 \quad e \quad(b-a) u+c v+M=0,
$$

cuja intersecção define os pontos umbílicos, são regulares e se intersectam transversalmente no $0=(0,0)$.

Associada a equação 1.11, consideremos a equação diferencial implícita, no espaço $\mathbb{R}^{2} \times \mathbb{P}_{1} \mathbb{R}$, definida na carta $(u, v, p)$ por,

$$
F(u, v, p)=[b v+L] p^{2}-[(b-a) u+c v+M] p-[b v+N]=0,
$$

onde $p=\frac{d y}{d x}$ e na carta $(u, v, q), q=\frac{d x}{d y}$, por

$$
G(u, v, q)=[b v+L]-[(b-a) u+c v+M] q-[b v+N] q^{2}=0 .
$$

Considerando o conjunto

$$
\mathbb{M}=\left\{(u, v,[u: v]) \in \mathbb{R}^{2} \times \mathbb{P}_{1} \mathbb{R}: F(u, v, p)=G(u, v, q)=0\right\}
$$

A condição de transversalidade é equivalente a dizer que $\mathbb{M}$ é regular em uma vizinhança da linha projetiva $\mathbb{P}_{1} \mathbb{R}$. De fato, na carta $(u, v, p)$ segue que no ponto $(0,0, p)$, temos:

$$
F_{u}=-(b-a) p=0, \quad F_{v}=b p^{2}-c p-b=0 \quad \text { e } \quad F_{p}=0
$$

então $p=0$ e $b=0$ ou $p \neq 0$ e $b=a$. Pela condição $\mathbf{T}$ nenhuma das duas é satisfeita.

Considere também a projeção $\pi: \mathbb{M} \rightarrow \mathbb{R}^{2}, \pi(u, v, p)=(u, v)$. O conjunto singular da projeção $\pi$ é definido por $F(u, v, p)=F_{p}(u, v, p)=0$ e coincide com a linha projetiva $\mathbb{P}_{1} \mathbb{R}$.

O campo de vetores $X=F_{p} \frac{\partial}{\partial u}+p F_{p} \frac{\partial}{\partial v}-\left(F_{u}+p F_{v}\right) \frac{\partial}{\partial p}$ está definido em $\mathbb{M}$ e a projeção das curvas integrais de $X$ são as soluções da equação diferencial implícita 1.11, com $q=\frac{d u}{d v} \neq 0$. Isso é facilmente verificado diferenciando a equação $F(u(t), v(t), p(t))=0$ com relação a $t$, ou seja, $F_{u} \dot{u}+F_{v} \dot{v}+F_{p} \dot{p}=0$. Então o campo 
de vetores $Y=\left(1, p,-\left(F_{u}+p F_{v}\right) / F_{p}\right), p=\frac{\dot{v}}{\dot{u}}$, esta bem definido em $\mathbb{M} \backslash\left\{F_{p}=0\right\}$. Multiplicando $Y$ por $F_{p}$ temos o campo de vetores $X=\left(F_{p}, p F_{p},-\left(F_{u}+p F_{v}\right)\right)$ que tem as mesmas curvas integrais de $Y$ em $\mathbb{M} \backslash \mathbb{P}_{1} \mathbb{R}$, além disso o conjunto singular de $\pi$ é somente a linha projetiva.

Os pontos singulares de $X$ na superfície implícita $\mathbb{M}$ são dados por: $\left(0,0, p_{i}\right)$, onde $p_{i}$ são as raízes da equação cúbica.

$$
h(p)=-p\left[b p^{2}-c p+(a-2 b)\right]=0,
$$

ou seja, os zeros de $-\left(F_{u}+p F_{v}\right)$. Os zeros do polinômio acima são dados por

$$
\begin{gathered}
p_{0}=0, \\
p_{1}=\frac{c}{2 b}-\sqrt{\left(\frac{c}{2 b}\right)^{2}-\frac{a}{b}+2} \mathrm{e} \\
p_{2}=\frac{c}{2 b}+\sqrt{\left(\frac{c}{2 b}\right)^{2}-\frac{a}{b}+2} .
\end{gathered}
$$

Eles representam as possíveis direções principais em que as linhas principais podem aproximar o ponto umbílico.

Mostremos agora que as singularidades de $X$, em $\mathbb{M}$, são de fato hiperbólicas, ou seja, as singularidades tem parte real diferente de zero, sobre as condições $\mathbf{T}$ e $\mathbf{D}$, que denominaremos de condições Darbouxianas.

Cálculos diretos mostram que

$$
D X(0,0, p)=\left(\begin{array}{ccc}
-(b-a) & 2 p b-c & 0 \\
-p(b-a) & 2 p^{2} b-p c & 0 \\
* & * & -3 b p^{2}+2 c p+(2 b-a)
\end{array}\right)
$$

Os autovalores de $D X(0,0, p)$ nas singularidades são dados por:

Na origem nós temos $\lambda_{t}=-(b-a)$ e $\lambda_{p}=(2 b-a)$, onde $\lambda_{t}$ é o autovalor associado ao autovetor transversal a linha projetiva e $\lambda_{p}$ esta associado a linha projetiva que é invariante pelo campo de vetores $X$.

Além disso se $\frac{a}{b}<1$ e $\frac{a}{b}>2$ a origem é um ponto de sela hiperbólico. Se $1<\frac{a}{b}<2$ a origem é um ponto hiperbólico nodal.

Para $p \neq 0$ os autovalores são dados por $\lambda_{t}=\left[2 b p^{2}-p c-(b-a)\right]$ e $\lambda_{p}=h^{\prime}(p)$, com isso temos que: 
Quando $\frac{a}{b}>\left(\frac{c}{2 b}\right)^{2}+2$ a origem é o único ponto singularidade de $X$, que é um ponto de sela hiperbólico.

Para $\frac{a}{b}<1$ nós temos três pontos de sela hiperbólicos.

Temos também que para $\left(\frac{c}{2 b}\right)^{2}+2>\frac{a}{b}>1, a \neq 2 b$, obtemos dois pontos de sela hiperbólicos e um ponto hiperbólico nodal.

Na carta $(x, y, q)$ o campo de vetores $Y=\left(q F_{p}, F_{p},-\left(q F_{u}+F_{v}\right)\right)$ é regular na vizinhança do ponto 0 e a análise deste campo é análoga ao do campo $X$.

Daí concluímos que a condição $\mathbf{D}$ é imposta para que as singularidades do campo $X$ e $Y$ sejam hiperbólicas.

Ao projetarmos as curvas integrais dos campos $X$ e $Y$, obtemos o comportamento das linhas de curvatura na vizinhança do ponto umbílico $p_{o}$.

Podemos agora enunciarmos o seguinte teorema que caracteriza a configuração dos pontos umbílicos Darbouxianos:

Teorema 4 (Gutierrez, Sotomayor,1982) Seja $p$ um ponto umbilico de uma imersão $\alpha$ dada nas coordenadas de Monge por:

$$
\alpha(u, v)=\left(u, v, \frac{k}{2}\left(u^{2}+v^{2}\right)+\frac{a}{6} u^{3}+\frac{b}{2} u^{2} v+\frac{c}{6} v^{3}+o(4)\right)
$$

Suponha as seguintes condições:

$$
\begin{gathered}
\mathrm{T}) \mathrm{b}(\mathrm{b}-\mathrm{a}) \neq 0 \\
\left.\left.\left.\mathrm{D}_{1}\right)\left(\frac{\mathrm{c}}{2 \mathrm{~b}}\right)^{2}-\frac{\mathrm{a}}{\mathrm{b}}+2<0 \quad \mathrm{D}_{2}\right)\left(\frac{\mathrm{c}}{2 \mathrm{~b}}\right)^{2}+2>\frac{\mathrm{a}}{\mathrm{b}}>1, \mathrm{a} \neq 2 \mathrm{~b} \quad \mathrm{D}_{3}\right) \frac{\mathrm{a}}{\mathrm{b}}<1
\end{gathered}
$$

Então o comportamento das linhas de curvatura entorno do ponto umbilico $p$, nos casos $\mathbf{D}_{\mathbf{1}}, \mathbf{D}_{2}$ e $\mathbf{D}_{3}$, chamados de Umbíticos Darbouxianos, é como na figura 1.5.

Logo a caracterização do tipos Darbouxianos depende do 3-jato da superfície, o qual determina o comportamento das linhas de curvatura em torno do ponto umbílico. Com o objetivo de caracterizar o tipo Darbouxiano dos pontos umbílicos de $C$, calcularemos o 3-jato da superfície de conflito em $p \in C$, em termos dos correspondentes 3-jatos das superfícies $B_{1}$ e $B_{2}$ em $p_{1}$ e $p_{2}$.

Então as superfícies convexas $B_{1}$ e $B_{2}$ são localmente dadas na carta de Monge $(x, y)$ e $(u, v)$ pelos gráficos de 


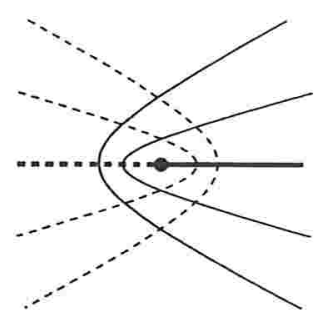

$D_{1}$

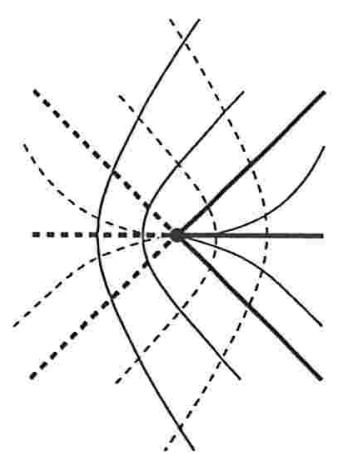

$D_{2}$

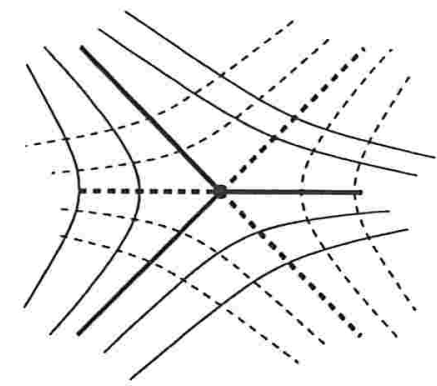

$D_{3}$

Figura 1.5: Umbílicos Darbouxianos

$$
\begin{aligned}
& f_{1}(x, y)=r+\frac{a}{2} x^{2}+\frac{b}{2} y^{2}+\frac{1}{6}\left(a_{30} x^{3}+3 a_{21} x^{2} y+3 a_{12} x y^{2}+a_{03} y^{3}+\ldots\right), \\
& f_{2}(u, v)=-r-\frac{a}{2} u^{2}-\frac{b}{2} v^{2}-\frac{1}{6}\left(b_{30} u^{3}+3 b_{21} u^{2} v+3 b_{12} u v^{2}+b_{03} v^{3}+\ldots\right),
\end{aligned}
$$

Seja $P=(X, Y, Z)$ um ponto do $\mathbb{R}^{3}$. Pelas considerações acima segue que $p=$ $(0,0,0) \in C$.

As expressões coordenadas dos jatos de segunda ordem em $p$ das projeções $\Pi_{1}(P)$ e $\Pi_{2}(P)$, com expressão nas coordenadas de Monge $(x, y)$ e $(u, v)$ serão calculadas agora.

A projeção $\Pi_{1}(X, Y, Z)=(x, y)$, cuja expressão coordenada, é definida implicitamente pelas equações

$$
\frac{\partial F}{\partial x}=\frac{\partial F}{\partial y}=0
$$

onde $F(x, y, X, Y, Z)=d\left(P, B_{1}\right)^{2}=(X-x)^{2}+(Y-y)^{2}+\left(Z-f_{1}(x, y)\right)^{2}$.

Usando o Teorema da Função Implícita, após cálculos extensos com auxílio do Maple, obtemos que solução do sistema de equações acima é dado por

$$
\begin{aligned}
x= & \frac{1}{1+r a} X-\frac{r a_{30}}{2(1+r a)^{3}} X^{2}-\frac{r a_{21}}{(1+r b)(1+r a)^{2}} X Y \\
& -\frac{r a_{12}}{2(1+r a)(1+b)^{2}} Y^{2}+\frac{a}{(1+r a)^{2}} X Z+\ldots \\
y= & \frac{1}{1+r b} Y-\frac{r a_{21}}{2(1+r b)(1+r a)^{2}} X^{2}-\frac{r a_{12}}{(1+r a)(1+r b)^{2}} X Y \\
& -\frac{r a_{30}}{2(1+b)^{3}} Y^{2}+\frac{b}{(1+r b)^{2}} Y Z+\ldots
\end{aligned}
$$


Similarmente, consideraremos a função

$$
G(x, y, X, Y, Z)=d\left(P, B_{2}\right)^{2}=(X-x)^{2}+(Y-y)^{2}+\left(Z-f_{2}(x, y)\right)^{2},
$$

obtemos que a projeção $\Pi_{2}(X, Y, Z)=(u, v)$, nas expressões coordenadas, é dada por

$$
\begin{aligned}
u= & \frac{1}{1+r a} X+\frac{r b_{30}}{2(1+r a)^{3}} X^{2}+\frac{r b_{21}}{(1+r b)(1+r a)^{2}} X Y \\
& +\frac{r b_{12}}{2(1+r a)(1+b)^{2}} Y^{2}-\frac{a}{(1+r a)^{2}} X Z+\ldots \\
v= & \frac{1}{1+r b} Y+\frac{r b_{21}}{2(1+r b)(1+r a)^{2}} X^{2}+\frac{r b_{12}}{(1+r a)(1+r b)^{2}} X Y \\
& +\frac{r b_{30}}{2(1+b)^{3}} Y^{2}-\frac{b}{(1+r b)^{2}} Y Z+\ldots
\end{aligned}
$$

Lembrando que a superfície de conflito $C$ é definida pela equação

$$
c(X, Y, Z)=d\left((X, Y, Z),\left(u, v, f_{2}(u, v)\right)\right)-d\left((X, Y, Z),\left(x, y, f_{1}(x, y)\right)\right)=0
$$

com $(x, y)$ e $(u, v)$ representando as projeções $\Pi_{1}(X, Y, Z)$ e $\Pi_{2}(X, Y, Z)$, dadas pelas expressões acima.

Um cálculo extenso nos leva para a seguinte expressão para o 3-jato da representação de Monge de $C$ na vizinhança de $p=(0,0,0)$ :

$$
\begin{aligned}
Z & =\frac{\left(a_{30}-b_{30}\right)}{2(1+a r)^{3}} \frac{X^{3}}{6}+\frac{\left(a_{21}-b_{21}\right)}{2(1+b r)(1+a r)^{2}} \frac{X^{2} Y}{2} \\
& +\frac{\left(a_{12}-b_{12}\right)}{2(1+a r)(1+b r)^{2}} \frac{X Y^{2}}{2}+\frac{\left(a_{03}-b_{03}\right)}{2(1+b r)^{3}} \frac{Y^{3}}{6}+\ldots
\end{aligned}
$$

Isto segue do Teorema da Função Implícita aplicado a função $c(X, Y, Z)$, observando que $c(0,0,0)=0$ e $\frac{\partial c}{\partial Z}(0,0,0)=4 r \neq 0$.

Como isto, podemos através do teorema de Gutierrez e Sotomayor demonstrado acima nesta seção, caracterizarmos o tipo Darbouxiano do umbílico que pode aparecer no ponto $p=(0,0,0)$. Observamos que da maneira que foi dada a expressão de $Z$, é preciso fazer uma rotação para eliminarmos o termo $X^{2} Y$, pois no teorema citado, a expressão não possui este termo. Note-se, entretanto, que quando $a_{21}=b_{21}, Z$ tem a forma reduzida acima. No trabalho de Darboux [2] existe uma caracterização dos pontos umbílicos Darbouxiano em função de todos os termos da parte cúbica, que pode ser aplicada diretamente a expressão de $Z$. 


\subsection{Exemplos de Conjuntos de Conflito}

Nesta seção daremos uma série de exemplos de conjuntos de conflito no $\mathbb{R}^{2}$ e $\mathbb{R}^{3}$. Daremos primeiramente alguns exemplos no $\mathbb{R}^{2}$ obtidos através de programas implementados no programa Mathematica 4; nestes pode-se obter uma seqüência satisfatória de pontos do conjunto de conflito, que nos indica o comportamento das curvas para certos conjuntos convexos do plano. Este método, já não é tão eficaz no $\mathbb{R}^{3}$ por causa dos fatores de visualização. Ao invés de termos pontos do conjunto de conflito, teremos curvas que ficam atrás das superfícies, logo é inviável ver o comportamento da superfície através deste método. No entanto, construímos alguns exemplos de superfícies de conflito através da obtenção da parametrização de tais superfícies.

\subsubsection{Curvas de Conflito}

O exemplos de curvas de conflito no plano são dados de duas maneiras: a primeira, usando exclusivamente algoritmos no programa Mathematica4, na qual obtemos uma descrição do comportamento das curvas de conflito através de alguns pontos desta curva, usando o deslocamento paralelo na direção da normal dos bordos dos conjuntos dados. Entretanto, não obtemos as parametrizações das curvas. A segunda, é mais completa pois chegamos nas parametrizações das curvas. Utilizando o método anterior para visualizar e verificar se a parametrização obtida passa pelos pontos da curva obtidos pelo primeiro método. Os exemplos obtidos pelo segundo método são elementares, pois o método fica inviável partir do momento em que não é possível isolar certas variáveis em função das outras, obtendo assim, funções implícitas como no caso da elipse, que não trataremos aqui.

A curva alpha no plano pode ser definida no Mathematica por

$$
\text { alpha[t] }:=\{\mathrm{a} 1[\mathrm{t}], \mathrm{a} 2[\mathrm{t}]\},
$$

onde a1 e a2 são funções de t. Nós podemos definir $\mathbf{J}$, o operador rotação dado por

$$
\mathbf{J}\left[\left\{\mathrm{p} 1_{-}, \mathrm{p} 2_{-}\right\}\right]:=\{-\mathrm{p} 2, \mathrm{p} 1\},
$$

que para cada vetor do plano dar um giro de $\pi / 2$ no sentido anti-horário.

A curva paralela a uma curva regular $\alpha$ é a curva plana dada por

$$
\text { parcurve }[\alpha][\mathbf{s}](\mathbf{t})=\alpha(\mathbf{t})+\frac{\mathbf{s} \mathbf{J} \alpha^{\prime}(\mathbf{t})}{\left\|\alpha^{\prime}(\mathbf{t})\right\|} \text {. }
$$


A versão Mathematica para esta definição é o operador parcuve dado por parcuve[alpha_] $\left[\mathrm{s}_{-}\right]\left[\mathrm{t}_{-}\right]:=$alpha[tt] $-\mathrm{sJ}[\mathrm{D}[\mathrm{alpha}[\mathrm{tt}], \mathrm{tt}]] /$ Sqrt $[$ Simplify[

$$
\mathrm{D}[\text { alpha[tt], tt].D[alpha[tt], tt]]]/.tt } \rightarrow \mathrm{t}
$$

O operador rotação de um ângulo $\theta$ é definido no Mathematica da seguinte forma

$$
\begin{aligned}
& \operatorname{rt}\left[\text { theta }_{-}, \text {alpha }_{-}\right]\left[\mathbf{t}_{-}\right]:=\{\operatorname{Cos}[\text { theta } \text { alpha }[\mathbf{t}][[1]]-\operatorname{Sin}[\text { theta }] \text { alpha }[\mathbf{t}][[2]], \\
& \operatorname{Sin}[\text { theta }] \text { alpha }[\mathbf{t}][[1]]+\operatorname{Cos}[\text { theta }] \text { alpha }[\mathbf{t}][[2]]\}
\end{aligned}
$$

A elipse será definida no Mathematica por

$$
\text { ellipse }\left[\mathbf{a}_{-}, \mathbf{b}_{-}\right]\left[\mathbf{t}_{-}\right]:=\{\mathrm{a} * \operatorname{Cos}[\mathbf{t}], \mathbf{b} * \operatorname{Sin}[\mathbf{t}]\}
$$

observamos que as circunferências estão contidas nestes casos para $a=b$.

Definiremos a função

$$
\begin{gathered}
\mathrm{p}\left[\mathrm{a}_{-}, \mathrm{b}_{-}, \mathrm{c}_{-}, \mathrm{d}_{-}\right]:=\text {ParametricPlot[Evaluate[Table[parcuve[ellipse[a, b]]][s]][t] } \\
+\{\mathrm{c}, \mathrm{d}\},\{\mathrm{s}, 0,6,0.5\}]],\{\mathrm{t}, 0,2 \mathrm{Pi}\}, \text { AspectRatio } \rightarrow \text { Automatic }]
\end{gathered}
$$

como sendo o operador do Mathematica que grafica, transladada para o ponto [c,d], a elipse[a,b] e suas curvas paralelas.

Assim sendo, podemos através do comando Show visualizar várias combinações destas figuras, como por exemplo

$$
\begin{aligned}
& \text { Show }[\mathrm{p}[3,1,-5,0], \mathrm{p}[1,3,5,0], \text { Axes } \rightarrow \text { False }] \text {; (ver figura 1.6) } \\
& \text { Show }[\mathrm{p}[2,2,-4,0], \mathrm{p}[1,3,5,0], \text { Axes } \rightarrow \text { False }] \text {; (ver figura 1.7) } \\
& \text { Show }[\mathrm{p}[1,3,-4,0], \mathrm{p}[1,3,4,0], \text { Axes } \rightarrow \text { False }] \text {; (ver figura 1.8) }
\end{aligned}
$$

Os pontos de intersecção entre as respectivas curvas paralelas são pontos da curvas de conflito, pois estão a uma mesma distância das elipses centrais. Ligando os pontos temos as curvas de conflito.

Outros exemplos podem ser obtidos através da função rotação, para isto serão definidas as funções

$$
\mathrm{r}[\mathrm{t}]:=\operatorname{rt}[\pi / 4, \text { ellipse }[3,1]][\mathrm{t}]
$$




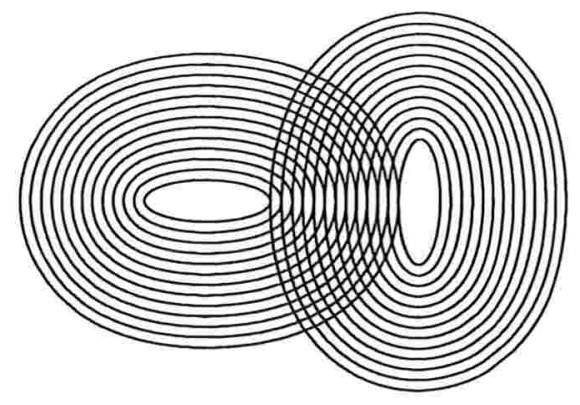

Figura 1.6: $\mathrm{p}[3, \mathbf{1},-5,0]$ e $\mathrm{p}[\mathbf{1}, 3,5,0]$

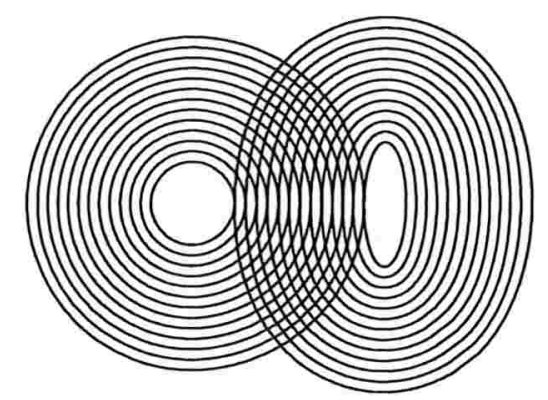

Figura 1.7: $\mathrm{p}[2,2,-4,0]$ e $\mathrm{p}[1,3,5,0]$

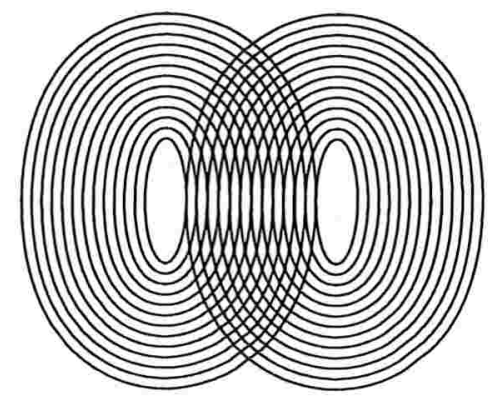

Figura 1.8: $\mathrm{p}[1,3,-4,0]$ e $\mathrm{p}[1,3,4,0]$

$$
\begin{gathered}
\mathrm{h}\left[\mathrm{c}_{-}, \mathrm{d}_{-}\right]:=\text {ParametricPlot }[\text { Evaluate}[\text { Table }[\text { parcuve }[\mathrm{r}][\mathrm{s}][\mathrm{t}]+\{\mathrm{c}, \mathrm{d}\}, \\
\{\mathrm{s}, 0,6,0.5\}]],\{\mathrm{t}, 0,2 \mathrm{Pi}\}, \text { AspectRatio } \rightarrow \text { Automatic }] .
\end{gathered}
$$

Daremos a seguir dois exemplos envolvendo rotações. Para obter estes exemplos podemos utilizar os seguintes comandos:

$$
\text { Show }[\mathrm{p}[3,1,-5,0], \mathrm{h}[6,0], \text { Axes } \rightarrow \text { False }] \text {; (ver figura 1.9) }
$$


Show $[\mathrm{h}[-5,0], \mathrm{h}[5,0]$, Axes $\rightarrow$ False $]$; (ver figura 1.10)

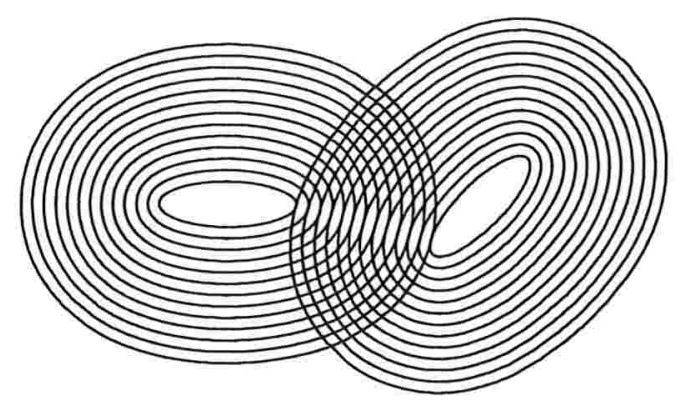

Figura 1.9: $\mathrm{p}[3,1,-5,0]$ e $\mathrm{h}[6,0]$

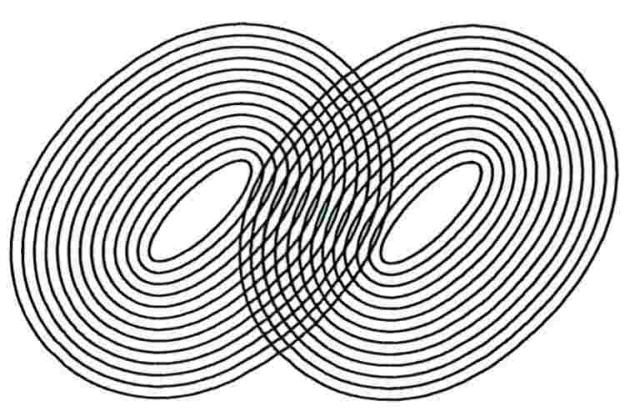

Figura 1.10: $\mathrm{h}[-5,0]$ e $\mathrm{h}[5,0]$

Nestes exemplos observa-se uma mudança no sinal da curvatura.

O próximo exemplo foi obtido chegando-se diretamente na expressão paramétrica da curva. Para isso igualamos as expressões das curvas paralelas a duas circunferência de raios diferentes. Sejam

$$
\begin{aligned}
& (x-6)^{2}+y^{2}=(5+t)^{2}, \\
& (x+6))^{2}+y^{2}=(1+t)^{2},
\end{aligned}
$$

as equações das curvas paralelas as circunferências de de raios 5 e 1, respectivamente, para $t \in \mathbb{R}$. Igualando as duas equações obtemos

$$
t=-3(1+x)
$$


substituindo na primeira equação acima, obtemos

$$
y^{2}=8\left(-4+x^{2}\right)
$$

Daí chegamos à parametrização para a curva de conflito, dada por

$$
c(y)=\left(\sqrt{\frac{y^{2}}{8}+4}, y\right)
$$

esta curva é definida no Mathematica como

$$
\mathrm{c}\left[\mathrm{y}_{-}\right]:=\left\{\operatorname{Sqrt}\left[\mathrm{y}^{2} / 8+4\right], \mathrm{y}\right\}
$$

para visualizar este gráfico e suas curvas geradoras podemos utilizar os seguintes comandos no Mathematica,

$$
\mathrm{cc}:=\text { ParametricPlot[Evaluate[c[y]], }\{\mathrm{y},-10,10\}]
$$

para definir o gráfico de $c\left[y_{-}\right] \mathrm{e}$

$$
\text { Show }[\{\mathbf{p}[5,5,-6,0], \mathrm{p}[1,1,6,0], \mathrm{cc}\}, \text { Axes } \rightarrow \text { False }] \text {; }
$$

para obter a figura 1.11 .

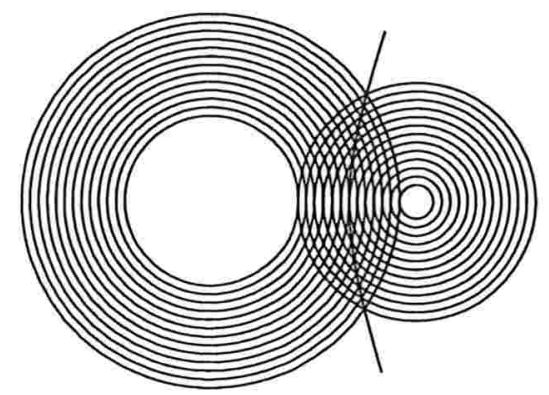

Figura 1.11: p[5,5,-6,0], p[1,1,6,0] e cc

É fácil ver, que caso este método seja utilizado para elipses, não teremos tamanha facilidade em isolar as variáveis para obtermos uma parametrização direta. Abaixo, segue a expressão em coordenadas para as curvas paralelas da elipse[a,b] em função de seno e coseno,

$$
\left(\cos (t)\left(a+\frac{b s}{\sqrt{b^{2} \cos ^{2}(t)+a^{2} \operatorname{sen}^{2}(t)}}\right), \operatorname{sen}(t)\left(b+\frac{a s}{\sqrt{b^{2} \cos ^{2}(t)+a^{2} \operatorname{sen}^{2}(t)}}\right)\right)
$$


onde $s$ é o parâmetro na direção da normal e $t$ é o parâmetro sobre a elipse.

Visualizando as figuras 1.6 a 1.8, podemos ser levados a acreditar que uma expressão de $x$ em função de $y$, bem maleável possa existir para parametrizarmos suas respectivas curvas de conflito, pois as curvas são tão simples quanto para duas circunferências. Trabalhamos nesta direção, porém devido a falta de tempo deixamos este estudo para abordarmos futuramente.

\subsubsection{Superfícies de Conflito}

A definição no Mathematica para superfície paralela é

$$
\begin{aligned}
& \operatorname{parsurf}\left[\mathrm{x}_{-}\right]\left[\mathrm{r}_{-}\right]\left[\mathrm{u}_{-}, \mathrm{v}_{-}\right]:= \operatorname{Module}[\{\mathrm{xu}, \mathrm{xv}, \mathrm{n} 1, \mathrm{n} 2, \mathrm{n} 3, \mathrm{n} 4\} \\
& \mathrm{xu}=\mathrm{D}[\mathrm{x}[\mathrm{uu}, \mathrm{vv}], \mathrm{uu}] ; \\
& \mathrm{xv}=\mathrm{D}[\mathrm{x}[\mathrm{uu}, \mathrm{vv}], \mathrm{vv}] ; \\
& \mathrm{n} 1=\operatorname{Cross}[\mathrm{xu}, \mathrm{xv}] / / \operatorname{Simplify} ; \\
& \mathrm{n} 2=\operatorname{Simplify}[\text { Factor[n1.n1]]; } \\
& \mathrm{n} 3=\operatorname{PowerExpand}[\operatorname{Sqrt}[\mathrm{n} 2]] / / \operatorname{Simplify} ; \\
& \mathrm{n} 4=\operatorname{Simplify}[\mathrm{n} 1 / \mathrm{n} 3] ; \\
&\text { Simplify[Together }[\mathrm{x}[\mathrm{uu}, \mathrm{vv}]+\mathrm{rn} 4]]] / .\{\mathrm{uu} \rightarrow \mathrm{u}, \mathrm{vv} \rightarrow \mathrm{v}\}
\end{aligned}
$$

Definiremos a esfera por

$$
\operatorname{esfera}\left[\mathbf{a}_{-}, \mathbf{b}_{-}\right]\left[\mathrm{u}_{-}, \mathrm{v}_{-}\right]:=\{\mathrm{a} \operatorname{Cos}[\mathrm{v}] \operatorname{Cos}[\mathrm{u}]+\mathrm{b}, \mathrm{aCos}[\mathrm{v}] \operatorname{Sin}[\mathrm{u}], \mathrm{aSin}[\mathrm{v}]\}
$$

onde o parâmetro a é o raio e b é o parâmetro de translação sobre o eixo $x$. Para para visualizar os exemplos usamos o parâmetro b para obter que os interiores das esferas sejam disjuntos.

No $\mathbb{R}^{3}$ não podemos usar o método do deslocamento paralelo ao longo da normal para visualizar as curvas de intersecção, como foi feito no $\mathbb{R}^{2}$ para visualizar os pontos de intersecção, pois existe o problema da dimensão. As superfícies paralelas tem dimensão dois, elas funcionam como paredes obstruindo a visualização das intersecções.

Logo, só usamos o segundo método, que é o de encontrar uma parametrização para a superfície de conflito e depois visualizá-la com as superfícies paralelas dos conjuntos que a geraram. 
Encontremos agora a parametrização para a superfície de conflito gerada por dois cones. A equação para os cones no Mathematica é

$$
\text { cone }\left[\mathbf{a}_{-}, \mathbf{b}_{-}\right]\left[\mathbf{t}_{-}, \mathbf{z}_{-}\right]:=\{\mathbf{a} * \operatorname{Cos}[\mathbf{t}]-\mathbf{b}, \mathbf{a} * \operatorname{Sin}[\mathbf{t}], \mathbf{z}\}
$$

Como para o caso de $\{a=5, b=-6\}$ e $\{a=1, b=6\}$ já temos a expressão para a curva de conflito das duas circunferências no plano $x y$, feito na seção anterior, basta então a.crescentarmos a variável $z$, da seguinte forma no Matehematica,

$$
\mathrm{c}\left[\mathrm{y}_{-}, \mathrm{z}_{-}\right]:=\left\{\operatorname{Sqrt}\left[\left(\mathrm{y}^{2}\right) / 8+4\right], \mathrm{y}, \mathrm{z}\right\}
$$

o gráfico desta curva será definido por

$$
\begin{gathered}
\mathrm{sc1}:=\text { ParametricPlot3D }[\text { Evaluate }[\mathrm{c}[\mathrm{y}, \mathrm{z}]],\{\mathrm{y},-10,10\},\{\mathrm{z},-10,10\}, \\
\text { Axes } \rightarrow \text { None, Boxed } \rightarrow \text { False }] ;
\end{gathered}
$$

e dos cones por

$\mathrm{c} 1:=$ ParametricPlot3D $[$ Evaluate $[$ cone $[5,-6][\mathbf{t}, \mathbf{z}]],\{\mathbf{t}, 0,2 \mathrm{Pi}\},\{\mathbf{z},-10,10\}$,

$$
\text { Axes } \rightarrow \text { None, Boxed } \rightarrow \text { False], }
$$

c2 := ParametricPlot3D[Evaluate[cone $[1,6][t, z]],\{t, 0,2 \mathrm{Pi}\},\{\mathrm{z},-10,10\}$,

$$
\text { Axes } \rightarrow \text { None, Boxed } \rightarrow \text { False]. }
$$

O comando para visualizar estes gráficos é

$$
\text { Show }[\{\mathrm{c} 1, \mathrm{c} 2, \mathrm{sc} 1\}, \text { Shading } \rightarrow \text { False }] \text {; }
$$

que nos dar a figura 1.12

O transporte paralelo de $c 1$ e $c 2$ é dado por

$$
\text { Pc1 := ParametricPlot3D }[\{c 1[u, v], \operatorname{parsurf}[\mathrm{c} 1][3][\mathrm{u}, \mathrm{v}], \operatorname{parsurf}[\mathrm{c} 1][4][\mathrm{u}, \mathrm{v}]
$$
parsurf $[\mathrm{c} 1][5][\mathrm{u}, \mathrm{v}]\} / /$ Evaluate, $\{\mathrm{u}, 0, \mathrm{Pi}\},\{\mathrm{v},-10,10\}$, PlotPoints $\rightarrow\{40,40\}$,

$$
\text { Axes } \rightarrow \text { None, Boxed } \rightarrow \text { False]; }
$$

$$
\operatorname{Pc} 2:=\text { ParametricPlot3D }[\{\mathrm{c} 2[\mathrm{u}, \mathrm{v}], \operatorname{parsurf}[\mathrm{c} 2][2][\mathrm{u}, \mathrm{v}], \operatorname{parsurf}[\mathrm{c} 2][3][\mathrm{u}, \mathrm{v}] \text {, }
$$
parsurf $[\mathrm{c} 2][4][\mathrm{u}, \mathrm{v}]\} / /$ Evaluate, $\{\mathrm{u}, 0, \mathrm{Pi}\},\{\mathrm{v},-10,10\}$, PlotPoints $\rightarrow\{40,40\}$,

$$
\text { Axes } \rightarrow \text { None, Boxed } \rightarrow \text { False]; }
$$




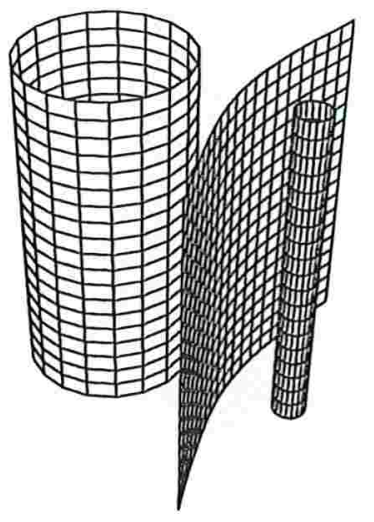

Figura 1.12: c1, c2 e sc1

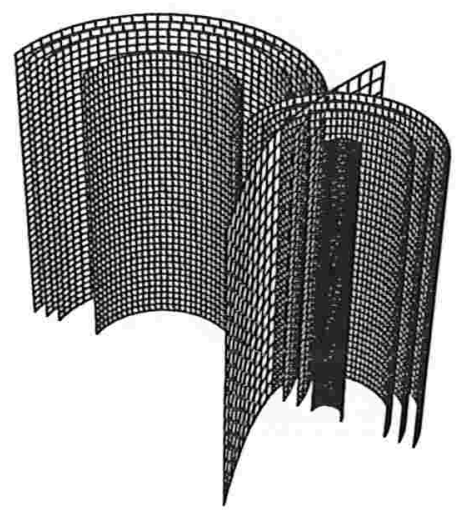

Figura 1.13: Pc1, Pc2 e sc1

Assim sendo, plotando $P c 1, P c 2$ e sc1 através do operador

$$
\text { Show }[\{\text { Pc1, Pc2, sc1 }\} \text {, Axes } \rightarrow \text { False, ViewPoint } \rightarrow\{-0.8,-2,3\}] \text {; }
$$

obtemos a figura 1.13 .

O próximo exemplo será a superfície de conflito entre duas esferas de mesmo raio. Para isto, igualamos as equações das seguintes esferas

$$
\begin{aligned}
& (x+4)^{2}+y^{2}+z^{2}=(2+t)^{2} \\
& (x-4)^{2}+y^{2}+z^{2}=(2+t)^{2}
\end{aligned}
$$


e fazendo cálculos semelhantes aos feitos no $\mathbb{R}^{2}$, obtemos $x=0$, ou seja o conflito será o plano $x y$. Logo as interseç̧ões das superfícies paralelas com o plano $x y$ são circunferências, ou seja, teremos circunferências gerando todo o plano $x y$ para $t \geq-2$.

A superfície de conflito é o plano $x y$, dado pela equação

$$
\mathrm{p}:=\{0,(2+\mathrm{t}) \operatorname{Cos}[\mathrm{v}],(2+\mathrm{t}) \operatorname{Sin}[\mathrm{v}]\}
$$

seu gráfico fica definido por

$\mathrm{sc} 2:=$ ParametricPlot3D $[$ Evaluate $[\mathrm{p}],\{\mathbf{t},-2,20\},\{\mathrm{v}, 0,2 \mathrm{Pi}\}$,

$$
\text { Axes, } \rightarrow \text { None, Boxed } \rightarrow \text { False]; }
$$

Para esboçarmos os gráficos das esferas, se faz necessário o uso dos seguintes comandos:

$\mathrm{e} 1:=$ ParametricPlot3D $[$ Evaluate $[\operatorname{esfera}[2,-4][\mathrm{u}, \mathrm{v}]],\{\mathrm{u}, 0,2 \mathrm{Pi}\},\{\mathrm{v},-\mathrm{Pi} / 2, \mathrm{Pi} / 2\}$

$$
\text { Axes } \rightarrow \text { None, Boxed } \rightarrow \text { False]; }
$$

$\mathrm{e} 2:=$ ParametricPlot3D $[$ Evaluate[esfera $[2,4][\mathrm{u}, \mathrm{v}]],\{\mathrm{u}, 0,2 \mathrm{Pi}\},\{\mathrm{v},-\mathrm{Pi} / 2, \mathrm{Pi} / 2\}$,

$$
\text { Axes } \rightarrow \text { None, Boxed } \rightarrow \text { False]; }
$$

As superfícies paralelas às esfera são dadas por

Pe1 := ParametricPlot3D $[\{$ esfera $[2,-4][\mathrm{u}, \mathrm{v}]$, parsurf $[\operatorname{esfera}[2,-4]][2][\mathrm{u}, \mathrm{v}]$, $\operatorname{parsurf}[\operatorname{espera}[2,-4]][3][\mathrm{u}, \mathrm{v}], \operatorname{parsurf}[\operatorname{espera}[2,-4]][4][\mathrm{u}, \mathrm{v}]\}$

//Evaluate, $\{\mathrm{u}, 0, \mathrm{Pi}\},\{\mathrm{v},-\mathrm{Pi} / 2, \mathrm{Pi} / 2\}$, PlotPoints $\rightarrow\{40,40\}$,

$$
\text { Axes } \rightarrow \text { None, Boxed } \rightarrow \text { False]; }
$$

Pe2 := ParametricPlot3D $[\{\operatorname{esfera}[2,4][\mathrm{u}, \mathrm{v}]$, parsurf $[$ esfera $[2,4]][2][\mathrm{u}, \mathrm{v}]$, parsurf $[\operatorname{espera}[2,4]][3][\mathrm{u}, \mathrm{v}]$, parsurf $[\operatorname{espera}[2,4]][4][\mathrm{u}, \mathrm{v}]\}$

//Evaluate, $\{\mathrm{u}, 0, \mathrm{Pi}\},\{\mathrm{v},-\mathrm{Pi} / 2, \mathrm{Pi} / 2\}$, PlotPoints $\rightarrow\{40,40\}$,

$$
\text { Axes } \rightarrow \text { None, Boxed } \rightarrow \text { False]; }
$$

Assim sendo, temos os comandos para visualizarmos a superfície de conflito primeiramente com os conjuntos geradores e depois com as superfícies paralelas

Show $[\{$ e1, e2, sc2 $\}$, Shading $\rightarrow$ False, ViewPoint $\rightarrow\{-0.4,-2,0\}$, (verfigura 1.14) 


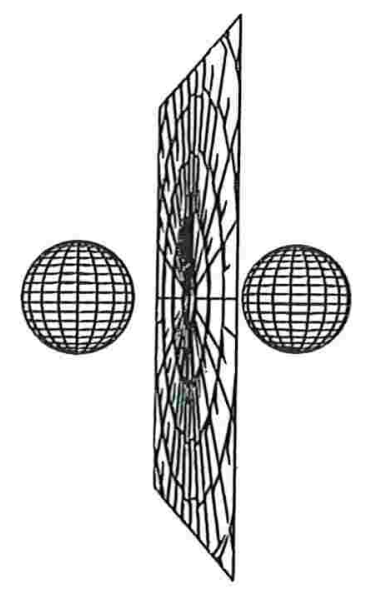

Figura 1.14: e1, e2 e sc2

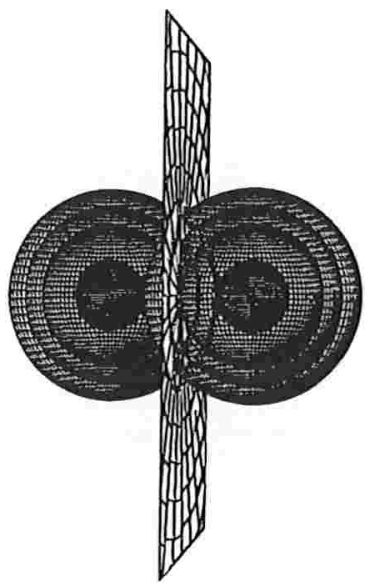

Figura 1.15: Pe1, Pe2 e sc2

Show $[\{$ Pe1, Pe2, sc2 $\}$, Axes $\rightarrow$ False, ViewPoint $\rightarrow\{0.4,-2,0\}$, (verfigura 1.15)

Igualando as equações das superfícies paralelas das esferas $[a=1, b=-6]$ e $[a=5$, $b=6]$, como foi feito para as esferas $[a=2, b=-4]$ e $[a=2, b=4]$, chegamos na equação paramétrica do conflito dada por

$$
c(y, z)=\left(\sqrt{\frac{\left(y^{2}+z^{2}\right)}{8}+4}, y, z\right)
$$


Sua representação no Mathematica é

$$
\mathrm{c}\left[\mathrm{y}_{-}, \mathrm{z}_{-}\right]:=\left\{\operatorname{Sqrt}\left[\left(\mathrm{y}^{2}+\mathrm{z}^{2}\right) / 8+4\right], \mathrm{y}, \mathrm{z}\right\}
$$

e seu gráfico fica definido por

$$
\begin{gathered}
\text { sc3 }:=\text { ParametricPlot3D }[\text { Evaluate }[\mathrm{c}],\{\mathrm{y},-10,10\},\{\mathrm{z},-10,10\}, \\
\text { Axes, } \rightarrow \text { None, Boxed } \rightarrow \text { False }] ; .
\end{gathered}
$$

Para visualizar as superfícies o processo é análogo, ao feito anteriormente, basta definir Pe3 e e 3 como Pe1 e e1 trocando os valores $a=2$ por $a=1$ e $b=-4$ por $b=-6$. Analogamente, definamos Pe4 e e 4 trocando $a=2$ por $a=5$ e $b=-4$ por $b=6$, obtendo como isto as figuras 1.16 e 1.17 .

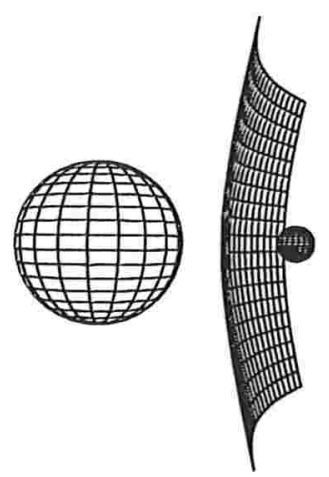

Figura 1.16: e3, e4 e sc3

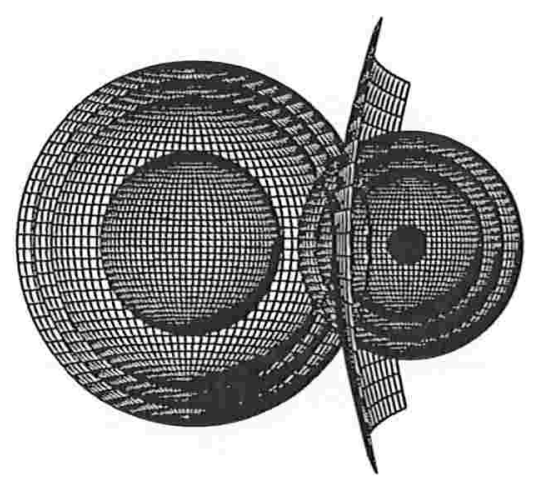

Figura 1.17: Pe3, Pe4 e sc3 


\section{Conjuntos de Conflito no $\mathbb{R}^{4}$}

\subsection{Introdução}

Neste capítulo desenvolveremos o estudo das hipersuperfícies de conflito no $\mathbb{R}^{4}$.

Consideraremos dois conjuntos $A_{1}$ e $A_{2}$ do $\mathbb{R}^{4}$ convexos, com interiores disjuntos e bordos $B_{i}$ de classe $C^{k}, k>1$, para definimos o conjunto de conflito a ser estudado.

A maioria das hipóteses feitas neste capítulo são herdadas do $\mathbb{R}^{3}$. Na primeira seção faremos uma breve introdução dos objetos que utilizaremos. A semelhança com o $\mathbb{R}^{3}$ é grande.

Um dos assuntos introduzidos é a definição de um referencial móvel $\left\{T_{1}, T_{2}, T_{3}, N\right\}$ sobre a hipersuperfície de conflito. Este por sua vez é dados em função dos bordos dos conjuntos $A_{i}$. A ligação entre o referencial e os $B_{i}$ nos possibilitará determinar a matriz $D N$ da derivada da normal a hipersuperfície de conflito.

O resultado principal é a matriz $D N$. Encontraremos uma expressão para a matriz na base $\left\{T_{1}, T_{2}, T_{3}\right\}$ do hiperplano tangente a hipersuperfície de conflito em um ponto $p \in C \backslash M$. Com isso, podemos encontrar uma expressão para as funções simétricas elementares em função dos autovalores desta matriz. As funções simétricas elementares nos fornecem características da geometria da hipersuperfície de conflito.

Por fim, obtemos alguns exemplos de hipersuperfícies de conflito. 


\subsection{Hipersuperfícies de Conflito do $\mathbb{R}^{4}$}

Sejam $A_{1}$ e $A_{2}$ dois conjuntos não vazios, fechados no espaço Euclidiano $\mathbb{R}^{4}$. Adotaremos a distância, o produto interno e a orientação canônicas do $\mathbb{R}^{4}$.

O conjunto de conflito $C\left(A_{1}, A_{2}\right)$ entre $A_{1}$ e $A_{2}$ é definido como no $\mathbb{R}^{3}$, ou seja,

$$
C\left(A_{1}, A_{2}\right)=\left\{p ; d\left(p, A_{1}\right)=d\left(p, A_{2}\right)\right\},
$$

onde $d(p, A)=\inf \{d(p, q) ; q \in A\}$.

É necessário, para obtermos hipersuperfícies regulares de classe $C^{k}, k \geq 1$, considerarmos somente o caso em que os $A_{i}$ são conjuntos fechados, convexos, com seus interiores disjuntos e bordos regulares $B_{i}=\partial A_{i}$ de classe $C^{k}, k \geq 2$.

Os bordos $B_{i}$ são orientados por campos diferenciáveis de vetores unitários $N_{i}$ normais as hipersuperfícies $B_{i}$. Assim, adotaremos a convenção que $N_{i}$ esta sempre apontando para o interior de $A_{i}$.

Denotaremos por $A_{i}^{r}$ o conjunto dos pontos a uma distância $r \geq 0$ de $A_{i}$; seu bordo é a superfície $B_{i}^{r}$ obtida pelo deslocamento de cada ponto $p_{i}$ de $B_{i}$ pela função $\sum_{i}^{r}\left(p_{i}\right)=p_{i}-r N_{i}\left(p_{i}\right)$. De acordo com as propriedades de vizinhança tubular, temos que $\Sigma_{i}^{r}$ é um difeomorfismo de classe $C^{k-1}$. (Ver p. 9, cap. 1)

Definiremos a função projeção $\Pi_{i}(p)=p_{i}$ de um ponto do conflito em $B_{i}$, caracterizada por

$$
d\left(p, A_{i}\right)=\left\langle\Pi_{i}(p)-p, N_{i}\left(\Pi_{i}(p)\right)\right\rangle,
$$

observamos que $\Pi_{i}$ pode ser definida como a inversa da função $\sum_{i}^{r}$. Temos então que $\Pi_{i}$ é uma função de classe $C^{k-1}$. Podemos também definir a projeção $\Pi_{i}^{r}: C \rightarrow B_{i}^{r}$, através da seguinte composição $\Pi_{i}^{r}=\Sigma_{i}^{r} \circ \Pi_{i}$.

Utilizando-se da função de classe $C^{k-1}$,

$$
c(p)=d\left(p, A_{2}\right)-d\left(p, A_{1}\right),
$$

para definir $C\left(A_{1}, A_{2}\right)$ como sendo o conjunto de nível zero, introduziremos um campo normal a hipersuperfície de conflito na direção do gradiente $\nabla c$, que é dado por

$$
\nabla c(p)=N_{1}\left(\Pi_{1}(p)\right)-N_{2}\left(\Pi_{i}(p)\right)
$$

A hipersuperfície de conflito, portanto, será orientada pela normal $N$ apontando de $A_{2}$ para $A_{1}$, dada por:

$$
N=\left|N_{1}\left(\Pi_{1}(p)\right)-N_{2}\left(\Pi_{2}(p)\right)\right|^{-1}\left[N_{1}\left(\Pi_{1}(p)\right)-N_{2}\left(\Pi_{2}(p)\right)\right] .
$$


Para simplificar a notação escreveremos $\nu(V)=|V|^{-1} V$ para a normalização de um vetor não nulo $V$. Logo

$$
N=\nu\left(N_{1}\left(\Pi_{1}(p)\right)-N_{2}\left(\Pi_{2}(p)\right)\right)
$$

Para introduzirmos um referencial móvel positivo em $C$ é necessário obter uma base do plano tangente. Um vetor tangente é

$$
T_{1}=\nu\left(N_{1}\left(\Pi_{1}(p)\right)+N_{2}\left(\Pi_{2}(p)\right)\right)
$$

Para completarmos a base do plano tangente precisamos de mais um vetor tangente, pois o terceiro é definido como o produto vetorial dos vetores tangentes já existentes e a normal.

Lema 1 Seja $W=\left\{w \in \mathbb{R}^{4} / w \in T B_{1}^{r}(p) \cap T B_{2}^{r}(p)\right\}, r=\operatorname{dist}\left(p, B_{i}\right)$. Então $w \in W$, se somente se, $w$ é ortogonal a $N$ e $T_{1}$ em $p$.

Prova. Se $w \in T B_{i}^{r}$ então $w$ é ortogonal a $N_{1}$ e $N_{2}$. Logo $w$ é ortogonal a $N$ e $T_{1}$. Se $w$ é ortogonal a $N$ e $T_{1}$ então temos que $\left.\langle N, w\rangle=<T_{1}, w\right\rangle=0$, daí $<N_{2}, w>=<N_{1}, w>=0$. Como $\Pi_{i}^{r}(p)=p$, para $r=\operatorname{dist}\left(p, B_{i}\right)$, e $T B_{i}^{r}(p)=\{v \in$ $\left.\mathbb{R}^{4} /<v, N_{i}>=0\right\}$ o vetor $w \in T B_{1}^{r}(p) \cap T B_{2}^{r}(p)$.

Como queremos um referencial ortonormal o vetor que estamos procurando pertence a $W$. Suponhamos que a projeção do vetor $D N\left(T_{1}\right)$ sobre o conjunto $W$ seja um vetor não nulo, ou seja, existe um vetor $w \in W, w \neq 0$, tal que $D N\left(T_{1}\right)-w$ é ortogonal a todo vetor de $W$. Denominaremos de $T_{2}$ o vetor unitário na direção da projeção $w$. Assim sendo, tomemos o último vetor da base, que denominaremos por $T_{3}$, como sendo $N \wedge T_{1} \wedge T_{2}$. Logo o referencial móvel será dado por $T_{1}, T_{2}, T_{3}, N$.

A hipersuperfície de conflito ficará dividida em dois conjuntos importantes a serem distinguidos. O primeiro é quando o campo $T_{1}$ não é singular. Neste caso, o conjunto $W$ tem dimensão dois. O segundo caso é quando o campo $T_{1}$ é singular. Neste caso, o conjunto $W$ tem dimensão três.

Note que o campo de vetores $T_{1}$ é singular nos pontos $p$ do conjunto fechado $M=M\left(A_{1}, A_{2}\right)$, onde $N_{1}\left(\Pi_{1}(p)\right)+N_{2}\left(\Pi_{2}(p)\right)=0$, que ocorre quando a distância de $C$ para $A_{i}$,

$$
r(p)=d\left(p, A_{1}\right)=d\left(p, A_{2}\right)
$$

é mínima, assumindo o valor $d_{m}=\frac{1}{2} d\left(A_{1}, A_{2}\right)$. A condição de convexidade estrita dos $B_{i}$ nos conjuntos $\Pi_{i}(M)$, reduz $M$ a um único ponto $p_{m}$. 
Considerando a convexidade estrita de $B_{i}$ em $p_{i}$, implica que $D N_{i}$ é um automorfismo do espaço tangente $T B_{i}$, com a identificação usual do espaço tangente $T B_{i}$ em $p_{i}$ com o da esfera unitária, em $N_{i}\left(p_{i}\right)$. Em termos das curvaturas principais $k_{1}^{i} \leq k_{2}^{i} \leq k_{3}^{i}$, que são os autovalores de $-D N_{i}$, a condição de convexidade estrita e a orientação do campo normal implica que $0<k_{1}^{i} \leq k_{2}^{i} \leq k_{3}^{i}$. Ver Rodrigues [10], Cap. 3, pag. 30 .

As funções simétricas elementares dos autovalores da matriz são as funções que aparecem como coeficientes do polinômio característico de uma matriz. Na próxima seção descreveremos como as funções simétricas elementares dos autovalores da matriz de $D N$, derivada da normal a superfície de conflito, podem ser escritas em função dos $B_{i}$ dados. As funções simétricas elementares das hipresuperfícies $B_{i}$ são obtidas dos autovalores da matriz $D N_{i}$ e suas expressões são dadas por,

$$
\begin{gathered}
\mathcal{K}^{i}=k_{1}^{i} k_{2}^{i} k_{3}^{i}=\operatorname{det}\left(-D N_{i}\right), \\
\mathcal{H}_{1}^{i}=\frac{1}{2}\left(k_{1}^{i} k_{2}^{i}+k_{1}^{i} k_{3}^{i}+k_{2}^{i} k_{3}^{i}\right) . \\
\mathcal{H}^{i}=\frac{1}{3}\left(k_{1}^{i}+k_{2}^{i}+k_{3}^{i}\right)=\frac{1}{3} \operatorname{traço}\left(-D N_{i}\right),
\end{gathered}
$$

onde $\mathcal{H}_{1}^{i}$ é a soma dos determinantes dos menores de $-D N_{i}$ e $k_{j}^{i}$ são os autovalores de $-D N_{i}$.

De acordo com a notação introduzida em Forsyth [3] (pag. 40 seção 281 capítulo XVI) temos que $\mathcal{H}^{i}$ pode ser chamada por curvatura linear, $\mathcal{H}_{1}^{i}$ de curvatura superficial e $\mathcal{K}^{i}$ a curvatura espacial (ou a curvatura volumétrica).

\subsection{Funções Simétricas Elementares}

Seja $p \in C \backslash M$ tal que suas projeções $p_{i}=\Pi_{i}(p)$ nos bordos $B_{i}$ sejam tais que os autovalores das matrizes $D N_{i}$ não sejam iguais, ou seja, $k_{1}^{i}<k_{2}^{i}<k_{3}^{i}$. Seja $\left\{E_{1}^{i}, E_{2}^{i}, E_{3}^{i}, N_{i}\right\}$ o referencial móvel positivo principal em $B_{i}$, numa vizinhança de $p_{i}$. Isto implica que

$$
D N_{i} \cdot E_{j}^{i}=-k_{j}^{i} E_{j}^{i}
$$

onde $k_{1}^{i}<k_{2}^{i}<k_{3}^{1}, i=1,2$, são as curvaturas principais de $B_{i}$. 
Sejam $R_{1}, R_{2}$ e $R_{3}$ as seguintes matrizes,

$$
\begin{aligned}
& R_{1}(\alpha):=\left(\begin{array}{ccc}
\cos (\alpha) & \operatorname{sen}(\alpha) & 0 \\
-\operatorname{sen}(\alpha) & \cos (\alpha) & 0 \\
0 & 0 & 1
\end{array}\right), \\
& R_{2}(\theta):=\left(\begin{array}{ccc}
\cos (\theta) & 0 & -\operatorname{sen}(\theta) \\
0 & 1 & 0 \\
\operatorname{sen}(\theta) & 0 & \cos (\theta)
\end{array}\right), \\
& R_{3}(\varphi):=\left(\begin{array}{ccc}
1 & 0 & 0 \\
0 & \cos (\varphi) & \operatorname{sen}(\varphi) \\
0 & -\operatorname{sen}(\varphi) & \cos (\varphi)
\end{array}\right) .
\end{aligned}
$$

Seja $H$ a multiplicação das três matrizes acima e $G$ sua inversa (que é igual a sua transposta), ou seja

$$
\begin{gathered}
H(\alpha, \theta, \varphi):=R_{1}(\alpha) \cdot R_{2}(\theta) \cdot R_{3}(\varphi) \\
G(\alpha, \theta, \varphi):=H^{-1}(\alpha, \theta, \varphi)=H^{\top}(\alpha, \theta, \varphi) .
\end{gathered}
$$

Logo,

$H=\left(\begin{array}{ccc}\cos \alpha \cos \theta & \operatorname{sen} \alpha \cos \varphi+\cos \alpha \operatorname{sen} \theta \operatorname{sen} \varphi & \operatorname{sen} \alpha \operatorname{sen} \varphi-\cos \alpha \operatorname{sen} \theta \cos \varphi \\ -\operatorname{sen} \alpha \cos \theta & \cos \alpha \cos \varphi-\operatorname{sen} \alpha \operatorname{sen} \theta \operatorname{sen} \varphi & \cos \alpha \operatorname{sen} \varphi+\operatorname{sen} \alpha \operatorname{sen} \theta \cos \varphi \\ \operatorname{sen} \theta & -\cos \theta \operatorname{sen} \varphi & \cos \theta \cos \varphi\end{array}\right)$,

e

$G=\left(\begin{array}{ccc}\cos \alpha \cos \theta & -\operatorname{sen} \alpha \cos \theta & \operatorname{sen} \theta \\ \operatorname{sen} \alpha \cos \varphi+\cos \alpha \operatorname{sen} \theta \operatorname{sen} \varphi & \cos \alpha \cos \varphi-\operatorname{sen} \alpha \operatorname{sen} \theta \operatorname{sen} \varphi & -\cos \theta \operatorname{sen} \varphi \\ \operatorname{sen} \alpha \operatorname{sen} \varphi-\cos \alpha \operatorname{sen} \theta \cos \varphi & \cos \alpha \operatorname{sen} \varphi+\operatorname{sen} \alpha \operatorname{sen} \theta \cos \varphi & \cos \theta \cos \varphi\end{array}\right)$.

Utilizaremos as matrizes acima para relacionar a base $\left\{E_{1}^{i}, E_{2}^{i}, E_{3}^{i}\right\}$ e a base $\left\{F_{1}^{i}=\right.$ $\left.\nu\left(D \Pi_{i}(p) \cdot T_{1}\right), F_{2}^{i}=\nu\left(D \Pi_{i}(p) \cdot T_{2}\right), F_{3}^{i}=\nu\left(D \Pi_{i}(p) \cdot T_{3}\right)\right\}$, dos espaços tangentes a $B_{i}$. Isso é feito através da matriz $H$, onde os ângulos envolvidos, também chamados de ângulos de Euller, são aqueles que através das matrizes do grupo de rotação $R_{1}, R_{2}$ e $R_{3}$ levam uma base na outra, da seguinte forma.

$$
\left(\begin{array}{c}
F_{1}^{i} \\
F_{2}^{i} \\
F_{3}^{i}
\end{array}\right)=H\left(\alpha_{i}, \theta_{i}, \varphi_{i}\right) \cdot\left(\begin{array}{c}
E_{1}^{i} \\
E_{2}^{i} \\
E_{3}^{i}
\end{array}\right)
$$


Como os vetores $F_{j}^{i}$ são definidos em função dos $T_{i}$, isso nos permitirá associar o hiperplano tangente da hipersuperfície de conflito e o hiperplano tangente a $B_{i}$. É através desta associação que conseguiremos obter a matriz de $D N$ do conflito em função dos dados das hipersuperfícies $B_{i}$.

Definamos as seguintes relações

$$
\begin{aligned}
& k_{n_{1}}^{i}=k_{n_{1}}^{i}\left(\alpha_{i}, \theta_{i}, \varphi_{i}\right)=k_{n_{1}}^{i}\left(F_{1}^{i}\right)=-\left\langle D N_{i}\left(F_{1}^{i}\right), F_{1}^{i}\right\rangle \\
& =k_{1}^{i} \cos ^{2}\left(\alpha_{i}\right) \cos ^{2}\left(\theta_{i}\right)+k_{2}^{i}\left[\operatorname{sen}\left(\alpha_{i}\right) \cos \left(\varphi_{i}\right)+\cos \left(\alpha_{i}\right) \operatorname{sen}\left(\theta_{i}\right) \operatorname{sen}\left(\varphi_{i}\right)\right]^{2} \\
& +k_{3}^{i}\left[\operatorname{sen}\left(\alpha_{i}\right) \operatorname{sen}\left(\varphi_{i}\right)-\cos \left(\alpha_{i}\right) \operatorname{sen}\left(\theta_{i}\right) \cos \left(\varphi_{i}\right)\right]^{2} \\
& k_{n_{2}}^{i}=k_{n_{2}}^{i}\left(\alpha_{i}, \theta_{i}, \varphi_{i}\right)=k_{n_{2}}^{i}\left(F_{2}^{i}\right)=-\left\langle D N_{i}\left(F_{2}^{i}\right), F_{2}^{i}\right\rangle \\
& =k_{1}^{i} \operatorname{sen}^{2}\left(\alpha_{i}\right) \cos ^{2}\left(\theta_{i}\right)+k_{2}^{i}\left[\cos \left(\alpha_{i}\right) \cos \left(\varphi_{i}\right)-\operatorname{sen}\left(\alpha_{i}\right) \operatorname{sen}\left(\theta_{i}\right) \operatorname{sen}\left(\varphi_{i}\right)\right]^{2} \\
& +k_{3}^{i}\left[\cos \left(\alpha_{i}\right) \operatorname{sen}\left(\varphi_{i}\right)+\operatorname{sen}\left(\alpha_{i}\right) \operatorname{sen}\left(\theta_{i}\right) \cos \left(\varphi_{i}\right)\right]^{2} \\
& k_{n_{3}}^{i}=k_{n_{3}}^{i}\left(\alpha_{i}, \theta_{i}, \varphi_{i}\right)=k_{n_{3}}^{i}\left(F_{3}^{i}\right)=-\left\langle D N_{i}\left(F_{3}^{i}\right), F_{3}^{i}\right\rangle \\
& =k_{1}^{i} \operatorname{sen}^{2}\left(\theta_{i}\right)+k_{2}^{i} \cos ^{2}\left(\theta_{i}\right) \operatorname{sen}^{2}\left(\varphi_{i}\right)+k_{3}^{i} \cos ^{2}\left(\theta_{i}\right) \cos ^{2}\left(\varphi_{i}\right) \\
& \tau_{12}^{i}=\tau_{12}^{i}\left(\alpha_{i}, \theta_{i}, \varphi_{i}\right)=\tau_{12}^{i}\left(F_{1}^{i}\right)=-\left\langle D N_{i}\left(F_{1}^{i}\right), F_{2}^{i}\right\rangle \\
& =-k_{1}^{i} \cos \left(\alpha_{i}\right) \cos ^{2}\left(\theta_{i}\right) \operatorname{sen}\left(\alpha_{i}\right)+k_{2}^{i}\left[\operatorname{sen}\left(\alpha_{i}\right) \cos \left(\varphi_{i}\right)+\cos \left(\alpha_{i}\right) \operatorname{sen}\left(\theta_{i}\right) \operatorname{sen}\left(\varphi_{i}\right)\right] \\
& {\left[\cos \left(\alpha_{i}\right) \cos \left(\varphi_{i}\right)-\operatorname{sen}\left(\alpha_{i}\right) \operatorname{sen}\left(\theta_{i}\right) \operatorname{sen}\left(\varphi_{i}\right)\right]+k_{3}^{i}\left[\operatorname{sen}\left(\alpha_{i}\right) \operatorname{sen}\left(\varphi_{i}\right)\right.} \\
& \left.-\cos \left(\alpha_{i}\right) \operatorname{sen}\left(\theta_{i}\right) \cos \left(\varphi_{i}\right)\right]\left[\cos \left(\alpha_{i}\right) \operatorname{sen}\left(\varphi_{i}\right)+\operatorname{sen}\left(\alpha_{i}\right) \operatorname{sen}\left(\theta_{i}\right) \cos \left(\varphi_{i}\right)\right](2.4) \\
& \tau_{13}^{i}=\tau_{13}^{i}\left(\alpha_{i}, \theta_{i}, \varphi_{i}\right)=\tau_{13}^{i}\left(F_{1}^{i}\right)=-\left\langle D N_{i}\left(F_{1}^{i}\right), F_{3}^{i}\right\rangle \\
& =k_{1}^{i} \cos \left(\alpha_{i}\right) \cos \left(\theta_{i}\right) \operatorname{sen}\left(\theta_{i}\right)-k_{2}^{i} \cos \left(\theta_{i}\right) \operatorname{sen}\left(\varphi_{i}\right)\left[\operatorname{sen}\left(\alpha_{i}\right) \cos \left(\varphi_{i}\right)\right. \\
& \left.+\cos \left(\alpha_{i}\right) \operatorname{sen}\left(\theta_{i}\right) \operatorname{sen}\left(\varphi_{i}\right)\right]+k_{3}^{i} \cos \left(\theta_{i}\right) \cos \left(\varphi_{i}\right)\left[\operatorname{sen}\left(\alpha_{i}\right) \operatorname{sen}\left(\varphi_{i}\right)\right. \\
& \left.-\cos \left(\alpha_{i}\right) \operatorname{sen}\left(\theta_{i}\right) \cos \left(\varphi_{i}\right)\right] \\
& \tau_{23}^{i}=\tau_{23}^{i}\left(\alpha_{i}, \theta_{i}, \varphi_{i}\right)=\tau_{23}^{i}\left(F_{2}^{i}\right)=-\left\langle D N_{i}\left(F_{2}^{i}\right), F_{3}^{i}\right\rangle \\
& =-k_{1}^{i} \cos \left(\theta_{i}\right) \operatorname{sen}\left(\alpha_{i}\right) \operatorname{sen}\left(\theta_{i}\right)-k_{2}^{i} \cos \left(\theta_{i}\right) \operatorname{sen}\left(\varphi_{i}\right)\left[\cos \left(\alpha_{i}\right) \cos \left(\varphi_{i}\right)\right. \\
& \left.-\operatorname{sen}\left(\alpha_{i}\right) \operatorname{sen}\left(\theta_{i}\right) \operatorname{sen}\left(\varphi_{i}\right)\right]+k_{3}^{i} \cos \left(\theta_{i}\right) \cos \left(\varphi_{i}\right)\left[\cos \left(\alpha_{i}\right) \operatorname{sen}\left(\varphi_{i}\right)\right. \\
& \left.+\operatorname{sen}\left(\alpha_{i}\right) \operatorname{sen}\left(\theta_{i}\right) \cos \left(\varphi_{i}\right)\right]
\end{aligned}
$$

Lema 2 Seja $I_{k}$ a matriz diagonal $3 \times 3$, na qual, os $a_{j j}=-k_{j}^{i}$. Então a matriz $H\left(\alpha_{i}, \theta_{i}, \varphi_{i}\right) . I_{k} . G\left(\alpha_{i}, \theta_{i}, \varphi_{i}\right)$ é dada por

$$
\left(\begin{array}{ccc}
-k_{n_{1}}^{i} & -\tau_{12}^{i} & -\tau_{13}^{i} \\
-\tau_{12}^{i} & -k_{n_{2}}^{i} & -\tau_{23}^{i} \\
-\tau_{13}^{i} & -\tau_{23}^{i} & -k_{n_{3}}^{i}
\end{array}\right)
$$


Prova. Fazendo a multiplicação $I_{k} \cdot G\left(\alpha_{i}, \theta_{i}, \varphi_{i}\right)$, obtemos:

$\left(\begin{array}{ccc}-k_{1}^{i} \cos \alpha \cos \theta & k_{1}^{i} \operatorname{sen} \alpha \cos \theta & -k_{1}^{i} \operatorname{sen} \theta \\ -k_{2}^{i}(\operatorname{sen} \alpha \cos \varphi+\cos \alpha \operatorname{sen} \theta \operatorname{sen} \varphi) & -k_{2}^{i}(\cos \alpha \cos \varphi-\operatorname{sen} \alpha \operatorname{sen} \theta \operatorname{sen} \varphi) & k_{2}^{i} \cos \theta \operatorname{sen} \varphi \\ -k_{3}^{i}(\operatorname{sen} \alpha \operatorname{sen} \varphi-\cos \alpha \operatorname{sen} \theta \cos \varphi) & -k_{3}^{i}(\cos \alpha \operatorname{sen} \varphi+\operatorname{sen} \alpha \operatorname{sen} \theta \cos \varphi) & -k_{3}^{i} \cos \theta \cos \varphi\end{array}\right)$

Daí temos que os elementos $\left(a_{1 j}\right)$ da matriz $H\left(\alpha_{i}, \theta_{i}, \varphi_{i}\right) \cdot I_{k} \cdot G\left(\alpha_{i}, \theta_{i}, \varphi_{i}\right)$ são dados por:

$$
\begin{gathered}
a_{11}=-k_{1}^{i} \cos ^{2}\left(\alpha_{i}\right) \cos ^{2}\left(\theta_{i}\right)-k_{2}^{i}\left[\operatorname{sen}\left(\alpha_{i}\right) \cos \left(\varphi_{i}\right)-\cos \left(\alpha_{i}\right) \operatorname{sen}\left(\theta_{i}\right) \operatorname{sen}\left(\varphi_{i}\right)\right]^{2} \\
-k_{3}^{i}\left[\operatorname{sen}\left(\alpha_{i}\right) \operatorname{sen}\left(\varphi_{i}\right)-\cos \left(\alpha_{i}\right) \operatorname{sen}\left(\theta_{i}\right) \cos \left(\varphi_{i}\right)\right]^{2} \\
a_{12}=k_{1}^{i} \cos \left(\alpha_{i}\right) \cos ^{2}\left(\theta_{i}\right) \operatorname{sen}\left(\alpha_{i}\right)-k_{2}^{i}\left[\operatorname{sen}\left(\alpha_{i}\right) \cos \left(\varphi_{i}\right)+\cos \left(\alpha_{i}\right) \operatorname{sen}\left(\theta_{i}\right) \operatorname{sen}\left(\varphi_{i}\right)\right] \\
{\left[\cos \left(\alpha_{i}\right) \cos \left(\varphi_{i}\right)-\operatorname{sen}\left(\alpha_{i}\right) \operatorname{sen}\left(\theta_{i}\right) \operatorname{sen}\left(\varphi_{i}\right)\right]-k_{3}^{i}\left[\operatorname{sen}\left(\alpha_{i}\right) \operatorname{sen}\left(\varphi_{i}\right)\right.} \\
\left.-\cos \left(\alpha_{i}\right) \operatorname{sen}\left(\theta_{i}\right) \cos \left(\varphi_{i}\right)\right]\left[\cos \left(\alpha_{i}\right) \operatorname{sen}\left(\varphi_{i}\right)+\operatorname{sen}\left(\alpha_{i}\right) \operatorname{sen}\left(\theta_{i}\right) \cos \left(\varphi_{i}\right)\right] \\
a_{13}=-k_{1}^{i} \cos \left(\alpha_{i}\right) \cos \left(\theta_{i}\right) \operatorname{sen}\left(\theta_{i}\right)+k_{2}^{i} \cos \left(\theta_{i}\right) \operatorname{sen}\left(\varphi_{i}\right)\left[\operatorname{sen}\left(\alpha_{i}\right) \cos \left(\varphi_{i}\right)\right. \\
\left.+\cos \left(\alpha_{i}\right) \operatorname{sen}\left(\theta_{i}\right) \operatorname{sen}\left(\varphi_{i}\right)\right]-k_{3}^{i} \cos \left(\theta_{i}\right) \cos \left(\varphi_{i}\right)\left[\operatorname{sen}\left(\alpha_{i}\right) \operatorname{sen}\left(\varphi_{i}\right)\right. \\
\left.-\cos \left(\alpha_{i}\right) \operatorname{sen}\left(\theta_{i}\right) \cos \left(\varphi_{i}\right)\right] .
\end{gathered}
$$

Analogamente pode-se verificar que $a_{2 j}=\left\langle D N_{i}\left(F_{j}^{i}\right), F_{2}^{i}\right\rangle$ e que $a_{3 j}=\left\langle D N_{i}\left(F_{j}^{i}\right), F_{3}^{i}\right\rangle$, ou seja,

$$
H\left(\alpha_{i}, \theta_{i}, \varphi_{i}\right) \cdot I_{k} \cdot G\left(\alpha_{i}, \theta_{i}, \varphi_{i}\right)=\left(\begin{array}{ccc}
\left\langle D N_{i}\left(F_{1}^{i}\right), F_{1}^{i}\right\rangle & \left\langle D N_{i}\left(F_{1}^{i}\right), F_{2}^{i}\right\rangle & \left\langle D N_{i}\left(F_{1}^{i}\right), F_{3}^{i}\right\rangle \\
\left\langle D N_{i}\left(F_{1}^{i}\right), F_{2}^{i}\right\rangle & \left\langle D N_{i}\left(F_{2}^{i}\right), F_{2}^{i}\right\rangle & \left\langle D N_{i}\left(F_{2}^{i}\right), F_{3}^{i}\right\rangle \\
\left\langle D N_{i}\left(F_{1}^{i}\right), F_{3}^{i}\right\rangle & \left\langle D N_{i}\left(F_{2}^{i}\right), F_{3}^{i}\right\rangle & \left\langle D N_{i}\left(F_{3}^{i}\right), F_{3}^{i}\right\rangle
\end{array}\right) .
$$

De (2.1) - (2.6) temos o que queríamos.

As propriedades abaixo relacionadas são obtidas através de demonstrações análogas às feitas para o $\mathbb{R}^{3}$. Portanto, optamos por apenas mencioná-las.

Denotemos por $\phi$ o ângulo entre $N_{i}$ e $T_{1}$. Então

$$
\operatorname{sen} \phi=\frac{1}{2}\left|N_{1}\left(\Pi_{1}(p)\right)-N_{2}\left(\Pi_{2}(p)\right)\right|=\left\langle F_{1}^{1}, T_{1}\right\rangle=\left\langle F_{1}^{2}, T_{1}\right\rangle .
$$

A base $\left\{T_{1}, T_{2}, T_{3}\right\}$ em $C \backslash M$ é projetada ao longo da derivada de $\Pi_{i}$ na base ortonormal $\left\{F_{1}^{i}, F_{2}^{i}, F_{3}^{i}\right\}$ em $B_{i}$. Os vetores $\left\{F_{1}^{i}, F_{2}^{i}, F_{3}^{i}\right\}, i=1,2$, podem ser escritos da seguinte forma:

$$
\begin{gathered}
\left\{F_{1}^{1}=\nu\left(N_{2}-\left\langle N_{1}, N_{2}\right\rangle N_{1}\right), F_{2}^{1}=T_{2}, F_{3}^{1}=T_{3}\right\}, \\
\left\{F_{1}^{2}=\nu\left(-N_{1}-\left\langle N_{1}, N_{2}\right\rangle N_{2}\right), F_{2}^{2}=T_{2}, F_{3}^{2}=T_{3}\right\} .
\end{gathered}
$$


Observe que $T_{2}$ e $T_{3}$ pertencem a $W$, logo ao fazermos $D \Pi_{i}(p) \cdot T_{2}$ e $D \Pi_{i}(p) \cdot T_{3}$ não haverá alteração nas direções. Por isso temos a igualdade acima $F_{2}^{i}=T_{2}$ e $F_{3}^{i}=T_{3}$.

Além do mais,

$$
D \Pi_{i}^{r}\left(T_{1}\right)=\operatorname{sen} \phi F_{1}^{i} \quad D \Pi_{i}^{r}\left(T_{2}\right)=F_{2}^{i} \quad D \Pi_{i}^{r}\left(T_{3}\right)=F_{3}^{i}
$$

Os referenciais principais $\left\{E_{1}^{i}, E_{2}^{i}, E_{3}^{i}, N_{i}\right\}$ em $B_{i}$ são transladados ao longo da normal para os referenciais principais em $B_{i}^{r}$. Isso segue do fato que $\Sigma_{i}^{r}$ preserva os campos de direções principais. As curvaturas principais sofrem mudanças para $k_{j}^{i}(r)=\frac{k_{j}^{i}}{1-r k_{j}^{i}}$. Ver Hicks [6], pag. 36 seção 2.6 .

As expressões para $k_{n_{1}}^{i}, k_{n_{2}}^{i}, k_{n_{3}}, \tau_{12}^{i}, \tau_{13}^{i}$ e $\tau_{23}^{i}$, definidas de (2.1) à (2.6), são dadas em termos das curvaturas principais de $B_{i}$, podendo ser obviamente modificadas para valerem em $B_{i}^{r}$ e denotaremos respectivamente por $k_{n_{1}}^{i}(r), k_{n_{2}}^{i}(r), k_{n_{3}}^{i}(r), \tau_{12}^{i}(r), \tau_{13}^{i}(r)$ e $\tau_{23}^{i}(r)$. Portanto, trocando $k_{j}^{i}$ por $k_{j}^{i}(r)$ nas equações de $(2.1)-(2.6)$, temos:

$$
\begin{gathered}
k_{n_{1}}^{i}(r)=k_{1}^{i}(r) \cos ^{2}\left(\alpha_{i}\right) \cos ^{2}\left(\theta_{i}\right)+k_{2}^{i}(r)\left[\operatorname{sen}\left(\alpha_{i}\right) \cos \left(\varphi_{i}\right)+\cos \left(\alpha_{i}\right) \operatorname{sen}\left(\theta_{i}\right) \operatorname{sen}\left(\varphi_{i}\right)\right]^{2} \\
+k_{3}^{i}(r)\left[\operatorname{sen}\left(\alpha_{i}\right) \operatorname{sen}\left(\varphi_{i}\right)-\cos \left(\alpha_{i}\right) \operatorname{sen}\left(\theta_{i}\right) \cos \left(\varphi_{i}\right)\right]^{2}
\end{gathered}
$$

$$
\begin{gathered}
k_{n_{2}}^{i}(r)=k_{1}^{i}(r) \operatorname{sen}^{2}\left(\alpha_{i}\right) \cos ^{2}\left(\theta_{i}\right)+k_{2}^{i}(r)\left[\cos \left(\alpha_{i}\right) \cos \left(\varphi_{i}\right)-\operatorname{sen}\left(\alpha_{i}\right) \operatorname{sen}\left(\theta_{i}\right) \operatorname{sen}\left(\varphi_{i}\right)\right]^{2} \\
+k_{3}^{i}(r)\left[\cos \left(\alpha_{i}\right) \operatorname{sen}\left(\varphi_{i}\right)+\operatorname{sen}\left(\alpha_{i}\right) \operatorname{sen}\left(\theta_{i}\right) \cos \left(\varphi_{i}\right)\right]^{2}
\end{gathered}
$$

$$
k_{n_{3}}^{i}(r)=k_{1}^{i}(r) \operatorname{sen}^{2}\left(\theta_{i}\right)+k_{2}^{i}(r) \cos ^{2}\left(\theta_{i}\right) \operatorname{sen}^{2}\left(\varphi_{i}\right)+k_{3}^{i}(r) \cos ^{2}\left(\theta_{i}\right) \cos ^{2}\left(\varphi_{i}\right)
$$

$$
\begin{aligned}
\tau_{12}^{i}(r)=- & k_{1}^{i}(r) \cos \left(\alpha_{i}\right) \cos ^{2}\left(\theta_{i}\right) \operatorname{sen}\left(\alpha_{i}\right)+k_{2}^{i}(r)\left[\operatorname{sen}\left(\alpha_{i}\right) \cos \left(\varphi_{i}\right)+\cos \left(\alpha_{i}\right) \operatorname{sen}\left(\theta_{i}\right) \operatorname{sen}\left(\varphi_{i}\right)\right] \\
& {\left[\cos \left(\alpha_{i}\right) \cos \left(\varphi_{i}\right)-\operatorname{sen}\left(\alpha_{i}\right) \operatorname{sen}\left(\theta_{i}\right) \operatorname{sen}\left(\varphi_{i}\right)\right]+k_{3}^{i}(r)\left[\operatorname{sen}\left(\alpha_{i}\right) \operatorname{sen}\left(\varphi_{i}\right)\right.} \\
& \left.-\cos \left(\alpha_{i}\right) \operatorname{sen}\left(\theta_{i}\right) \cos \left(\varphi_{i}\right)\right]\left[\cos \left(\alpha_{i}\right) \operatorname{sen}\left(\varphi_{i}\right)+\operatorname{sen}\left(\alpha_{i}\right) \operatorname{sen}\left(\theta_{i}\right) \cos \left(\varphi_{i}\right)\right](2.12)
\end{aligned}
$$

$$
\begin{aligned}
\tau_{13}^{i}(r)=k_{1}^{i}(r) & \cos \left(\alpha_{i}\right) \cos \left(\theta_{i}\right) \operatorname{sen}\left(\theta_{i}\right)-k_{2}^{i}(r) \cos \left(\theta_{i}\right) \operatorname{sen}\left(\varphi_{i}\right)\left[\operatorname{sen}\left(\alpha_{i}\right) \cos \left(\varphi_{i}\right)\right. \\
+ & \left.\cos \left(\alpha_{i}\right) \operatorname{sen}\left(\theta_{i}\right) \operatorname{sen}\left(\varphi_{i}\right)\right]+k_{3}^{i}(r) \cos \left(\theta_{i}\right) \cos \left(\varphi_{i}\right)\left[\operatorname{sen}\left(\alpha_{i}\right) \operatorname{sen}\left(\varphi_{i}\right)\right. \\
& \left.-\cos \left(\alpha_{i}\right) \operatorname{sen}\left(\theta_{i}\right) \cos \left(\varphi_{i}\right)\right]
\end{aligned}
$$

$$
\begin{aligned}
\tau_{23}^{i}(r)=-k_{1}^{i}(r) \cos \left(\theta_{i}\right) \operatorname{sen}\left(\alpha_{i}\right) \operatorname{sen}\left(\theta_{i}\right)-k_{2}^{i}(r) \cos \left(\theta_{i}\right) \operatorname{sen}\left(\varphi_{i}\right)\left[\cos \left(\alpha_{i}\right) \cos \left(\varphi_{i}\right)\right. \\
\left.\quad-\operatorname{sen}\left(\alpha_{i}\right) \operatorname{sen}\left(\theta_{i}\right) \operatorname{sen}\left(\varphi_{i}\right)\right]+k_{3}^{i}(r) \cos \left(\theta_{i}\right) \cos \left(\varphi_{i}\right)\left[\cos \left(\alpha_{i}\right) \operatorname{sen}\left(\varphi_{i}\right)\right. \\
\left.\quad+\operatorname{sen}\left(\alpha_{i}\right) \operatorname{sen}\left(\theta_{i}\right) \cos \left(\varphi_{i}\right)\right]
\end{aligned}
$$


A seguir encontraremos uma expressão para a derivada da normal, $D N$, da hipersuperfície de conflito em função dos dados geométricos das hipersuperfícies $B_{i}$.

Proposição 2 Com a notação acima, nos pontos de $C \backslash M$, temos

$$
\begin{aligned}
& D N . T_{1}=\frac{\operatorname{sen} \phi}{2}\left[k_{n_{1}}^{2}(r)-k_{n_{1}}^{1}(r)\right] T_{1}+\frac{1}{2}\left[\tau_{12}^{2}(r)-\tau_{12}^{1}(r)\right] T_{2}+\frac{1}{2}\left[\tau_{13}^{2}(r)-\tau_{13}^{1}(r)\right] T_{3}, \\
& D N . T_{2}=\frac{1}{2}\left[\tau_{12}^{2}(r)-\tau_{12}^{1}(r)\right] T_{1}+\frac{1}{2 \operatorname{sen} \phi}\left[k_{n_{2}}^{2}(r)-k_{n_{2}}^{1}(r)\right] T_{2}+\frac{1}{2 \operatorname{sen} \phi}\left[\tau_{23}^{2}(r)-\tau_{23}^{1}(r)\right] T_{3}, \\
& D N . T_{3}=\frac{1}{2}\left[\tau_{13}^{2}(r)-\tau_{13}^{1}(r)\right] T_{1}+\frac{1}{2 \operatorname{sen} \phi}\left[\tau_{23}^{2}(r)-\tau_{23}^{1}(r)\right] T_{2}+\frac{1}{2 \operatorname{sen} \phi}\left[k_{n_{3}}^{2}(r)-k_{n_{3}}^{1}(r)\right] T_{3} .
\end{aligned}
$$

Prova. A conclusão segue dos cálculos dos produtos internos em

$$
\begin{aligned}
& D N\left(T_{1}\right)=\left\langle D N\left(T_{1}\right), T_{1}\right\rangle T_{1}+\left\langle D N\left(T_{1}\right), T_{2}\right\rangle T_{2}+\left\langle D N\left(T_{1}\right), T_{3}\right\rangle T_{3}, \\
& D N\left(T_{2}\right)=\left\langle D N\left(T_{2}\right), T_{1}\right\rangle T_{1}+\left\langle D N\left(T_{2}\right), T_{2}\right\rangle T_{2}+\left\langle D N\left(T_{2}\right), T_{3}\right\rangle T_{3} . \\
& D N\left(T_{3}\right)=\left\langle D N\left(T_{3}\right), T_{1}\right\rangle T_{1}+\left\langle D N\left(T_{3}\right), T_{2}\right\rangle T_{2}+\left\langle D N\left(T_{3}\right), T_{3}\right\rangle T_{3} .
\end{aligned}
$$

Diferenciando $N$, obtemos:

$$
D N=\frac{D N_{1} \cdot D \Pi_{1}^{r}-D N_{2} \cdot D \Pi_{2}^{r}}{2 \operatorname{sen} \phi}+\frac{1}{2} D\left[(\operatorname{sen} \phi)^{-1}\right] \cdot\left[N_{1}\left(\Pi_{1}(p)\right)-N_{2}\left(\Pi_{2}(p)\right)\right] .
$$

A contribuição do segundo termo nos produtos internos de (2.15), (2.16) e (2.17) é nula. Logo, consideraremos somente o primeiro termo de $D N$.

Lembrando que as bases $E_{j}^{i}$ e $F_{j}^{i}$ são relacionadas por

$$
\left(\begin{array}{c}
F_{1}^{i} \\
F_{2}^{i} \\
F_{3}^{i}
\end{array}\right)=H\left(\alpha_{i}, \theta_{i}, \varphi_{i}\right) \cdot\left(\begin{array}{c}
E_{1}^{i} \\
E_{2}^{i} \\
E_{3}^{i}
\end{array}\right) .
$$

Calculemos agora $D N_{i} D \Pi_{i}^{r}$ nas direções $T_{1}, T_{2}$ e $T_{3}$, para sabermos o valor de $D N$ nestas direções. De (2.8), obtemos

$$
\left(\begin{array}{c}
D N_{i} \cdot D \Pi_{i}^{r}\left(T_{1}\right) \\
D N_{i} \cdot D \Pi_{i}^{r}\left(T_{2}\right) \\
D N_{i} \cdot D \Pi_{i}^{r}\left(T_{3}\right)
\end{array}\right)=\left(\begin{array}{c}
D N_{i}\left(\operatorname{sen} \phi F_{1}^{i}\right) \\
D N_{i}\left(F_{2}^{i}\right) \\
D N_{i}\left(F_{3}^{i}\right)
\end{array}\right)=I_{\phi} \cdot\left(\begin{array}{c}
D N_{i}\left(F_{1}^{i}\right) \\
D N_{i}\left(F_{2}^{i}\right) \\
D N_{i}\left(F_{3}^{i}\right)
\end{array}\right)
$$

onde $I_{\phi}$ é a matriz diagonal $3 \times 3$, na qual, o $a_{11}=\operatorname{sen} \phi$ e $a_{22}=a_{33}=1$. Denotando por $h_{l j}$ os elementos da matriz $H\left(\alpha_{i}, \theta_{i}, \varphi_{i}\right)$, temos:

$$
=I_{\phi} \cdot\left(\begin{array}{c}
D N_{i}\left(h_{11} E_{1}^{i}+h_{12} E_{2}^{i}+h_{13} E_{3}^{i}\right) \\
D N_{i}\left(h_{21} E_{1}^{i}+h_{22} E_{2}^{i}+h_{23} E_{3}^{i}\right) \\
D N_{i}\left(h_{31} E_{1}^{i}+h_{32} E_{2}^{i}+h_{33} E_{3}^{i}\right)
\end{array}\right)
$$




$$
\begin{gathered}
=I_{\phi} \cdot\left(\begin{array}{c}
h_{11} D N_{i}\left(E_{1}^{i}\right)+h_{12} D N_{i}\left(E_{2}^{i}\right)+h_{13} D N_{i}\left(E_{3}^{i}\right) \\
h_{21} D N_{i}\left(E_{1}^{i}\right)+h_{22} D N_{i}\left(E_{2}^{i}\right)+h_{23} D N_{i}\left(E_{3}^{i}\right) \\
h_{31} D N_{i}\left(E_{1}^{i}\right)+h_{32} D N_{i}\left(E_{2}^{i}\right)+h_{33} D N_{i}\left(E_{3}^{i}\right)
\end{array}\right) \\
=I_{\phi} \cdot H\left(\alpha_{i}, \theta_{i}, \varphi_{i}\right) \cdot\left(\begin{array}{c}
D N_{i}\left(E_{1}^{i}\right) \\
D N_{i}\left(E_{2}^{i}\right) \\
D N_{i}\left(E_{3}^{i}\right)
\end{array}\right)=I_{\phi} \cdot H\left(\alpha_{i}, \theta_{i}, \varphi_{i}\right) \cdot\left(\begin{array}{c}
-k_{1}^{i}(r) E_{1}^{i} \\
-k_{2}^{i}(r) E_{2}^{i} \\
-k_{3}^{i}(r) E_{3}^{i}
\end{array}\right)
\end{gathered}
$$

Denotando por $I_{k}^{r}$ a matriz diagonal $3 \times 3$, na qual, os $a_{j j}=-k_{j}^{i}(r)$, temos

$$
=I_{\phi} \cdot H\left(\alpha_{i}, \theta_{i}, \varphi_{i}\right) \cdot I_{k}^{r} \cdot\left(\begin{array}{c}
E_{1}^{i} \\
E_{2}^{i} \\
E_{3}^{i}
\end{array}\right)=I_{\phi} \cdot H\left(\alpha_{i}, \theta_{i}, \varphi_{i}\right) \cdot I_{k}^{r} \cdot G\left(\alpha_{i}, \theta_{i}, \varphi_{i}\right) \cdot\left(\begin{array}{c}
F_{1}^{i} \\
F_{2}^{i} \\
F_{3}^{i}
\end{array}\right)
$$

Do Lema 2 e de (2.9)-(2.14), obtemos

$$
=I_{\phi} \cdot\left(\begin{array}{ccc}
-k_{n_{1}}^{i}(r) & -\tau_{12}^{i}(r) & -\tau_{13}^{i}(r) \\
-\tau_{12}^{i}(r) & -k_{n_{2}}^{i}(r) & -\tau_{23}^{i}(r) \\
-\tau_{13}^{i}(r) & -\tau_{23}^{i}(r) & -k_{n_{3}}^{i}(r)
\end{array}\right) \cdot\left(\begin{array}{c}
F_{1}^{i} \\
F_{2}^{i} \\
F_{3}^{i}
\end{array}\right)
$$

Daí, temos que:

$$
\left(\begin{array}{c}
D N_{i} \cdot D \Pi_{i}^{r}\left(T_{1}\right) \\
D N_{i} \cdot D \Pi_{i}^{r}\left(T_{2}\right) \\
D N_{i} \cdot D \Pi_{i}^{r}\left(T_{3}\right)
\end{array}\right)=I_{\phi} \cdot\left(\begin{array}{c}
-k_{n_{1}}^{i}(r) F_{1}^{i}-\tau_{12}^{i}(r) F_{2}^{i}-\tau_{13}^{i}(r) F_{3}^{i} \\
-\tau_{12}^{i}(r) F_{1}^{i}-k_{n_{2}}^{i}(r) F_{2}^{i}-\tau_{23}^{i}(r) F_{3}^{i} \\
-\tau_{13}^{i}(r) F_{1}^{i}-\tau_{23}^{i}(r) F_{2}^{i}-k_{n_{3}}^{i}(r) F_{3}^{i}
\end{array}\right)
$$

Denotando por $H I G(i)$ a última matriz acima, temos

$$
\left(\begin{array}{c}
D N_{i} \cdot D \Pi_{i}^{r}\left(T_{1}\right) \\
D N_{i} \cdot D \Pi_{i}^{r}\left(T_{2}\right) \\
D N_{i} \cdot D \Pi_{i}^{r}\left(T_{3}\right)
\end{array}\right)=I_{\phi} \cdot H I G(i)
$$

Considerando somente o primeiro termo de (2.18), obtemos

$$
\begin{gathered}
\left(\begin{array}{c}
D N\left(T_{1}\right) \\
D N\left(T_{2}\right) \\
D N\left(T_{3}\right)
\end{array}\right)=\frac{1}{2 \operatorname{sen} \phi}\left[\left(\begin{array}{c}
D N_{1} \cdot D \Pi_{1}^{r}\left(T_{1}\right) \\
D N_{1} \cdot D \Pi_{1}^{r}\left(T_{2}\right) \\
D N_{1} \cdot D \Pi_{1}^{r}\left(T_{3}\right)
\end{array}\right)-\left(\begin{array}{c}
D N_{2} \cdot D \Pi_{2}^{r}\left(T_{1}\right) \\
D N_{2} \cdot D \Pi_{2}^{r}\left(T_{2}\right) \\
D N_{2} \cdot D \Pi_{2}^{r}\left(T_{3}\right)
\end{array}\right)\right]+\cdots \\
=\frac{1}{2 \operatorname{sen} \phi} \cdot I_{\phi} \cdot(H I G(1)-H I G(2))+\cdots
\end{gathered}
$$


Logo,

onde $U$ é dada por

$$
\left(\begin{array}{l}
D N\left(T_{1}\right) \\
D N\left(T_{2}\right) \\
D N\left(T_{3}\right)
\end{array}\right)=\frac{1}{2 \operatorname{sen} \phi} \cdot U+\cdots
$$

$$
U=\left(\begin{array}{c}
\operatorname{sen} \phi\left\{\left[k_{n_{1}}^{2}(r) F_{1}^{2}-k_{n_{1}}^{1}(r) F_{1}^{1}\right]+\left[\tau_{12}^{2}(r) F_{2}^{2}-\tau_{12}^{1}(r) F_{2}^{1}\right]+\left[\tau_{13}^{2}(r) F_{3}^{2}-\tau_{13}^{1}(r) F_{3}^{1}\right]\right\} \\
{\left[\tau_{12}^{2}(r) F_{1}^{2}-\tau_{12}^{1}(r) F_{1}^{1}\right]+\left[k_{n_{2}}^{2}(r) F_{2}^{2}-k_{n_{2}}^{1}(r) F_{2}^{1}\right]+\left[\tau_{23}^{2}(r) F_{3}^{2}-\tau_{23}^{1}(r) F_{3}^{1}\right]} \\
{\left[\tau_{13}^{2}(r) F_{1}^{1}-\tau_{13}^{1}(r) F_{1}^{2}\right]+\left[\tau_{23}^{2}(r) F_{2}^{2}-\tau_{23}^{1}(r) F_{2}^{1}\right]+\left[k_{n_{3}}^{2}(r) F_{3}^{2}-k_{n_{3}}^{1}(r) F_{3}^{1}\right]}
\end{array}\right)
$$

Fazendo os produtos internos de (2.15) e utilizando (2.7), temos,

$$
\begin{aligned}
\left\langle D N\left(T_{1}\right), T_{1}\right\rangle & =\left\langle\frac{1}{2}\left\{\left[k_{n_{1}}^{2}(r) F_{1}^{2}-k_{n_{1}}^{1}(r) F_{1}^{1}\right]+\left[\tau_{12}^{2}(r) F_{2}^{2}-\tau_{12}^{1}(r) F_{2}^{1}\right]+\left[\tau_{13}^{2}(r) F_{3}^{2}-\tau_{13}^{1}(r) F_{3}^{1}\right]\right\}, T_{1}\right\rangle \\
& =\frac{1}{2}\left\{k_{n_{1}}^{2}(r)\left\langle F_{1}^{2}, T_{1}\right\rangle-k_{n_{1}}^{1}(r)\left\langle F_{1}^{1}, T_{1}\right\rangle\right\}=\frac{\operatorname{sen} \phi}{2}\left[k_{n_{1}}^{2}(r)-k_{n_{1}}^{1}(r)\right] \\
\left\langle D N\left(T_{1}\right), T_{2}\right\rangle & =\left\langle\frac{1}{2}\left\{\left[k_{n_{1}}^{2}(r) F_{1}^{2}-k_{n_{1}}^{1}(r) F_{1}^{1}\right]+\left[\tau_{12}^{2}(r) F_{2}^{2}-\tau_{12}^{1}(r) F_{2}^{1}\right]+\left[\tau_{13}^{2}(r) F_{3}^{2}-\tau_{13}^{1}(r) F_{3}^{1}\right]\right\}, T_{2}\right\rangle \\
& =\frac{1}{2}\left\{\tau_{12}^{2}(r)\left\langle F_{2}^{2}, T_{2}\right\rangle-\tau_{12}^{1}(r)\left\langle F_{2}^{1}, T_{2}\right\rangle\right\}=\frac{1}{2}\left[\tau_{12}^{2}(r)-\tau_{12}^{1}(r)\right] \\
\left\langle D N\left(T_{1}\right), T_{3}\right\rangle & =\left\langle\frac{1}{2}\left\{\left[k_{n_{1}}^{2}(r) F_{1}^{2}-k_{n_{1}}^{1}(r) F_{1}^{1}\right]+\left[\tau_{12}^{2}(r) F_{2}^{2}-\tau_{12}^{1}(r) F_{2}^{1}\right]+\left[\tau_{13}^{2}(r) F_{3}^{2}-\tau_{13}^{1}(r) F_{3}^{1}\right]\right\}, T_{3}\right\rangle \\
& =\frac{1}{2}\left\{\tau_{13}^{2}(r)\left\langle F_{3}^{2}, T_{3}\right\rangle-\tau_{13}^{1}(r)\left\langle F_{3}^{1}, T_{3}\right\rangle\right\}=\frac{1}{2}\left[\tau_{13}^{2}(r)-\tau_{13}^{1}(r)\right]
\end{aligned}
$$

Analogamente obtemos os produtos internos de (2.16) e (2.17).

De posse da matriz $D N$, podemos obter as expressões para as funções simétricas elementares das curvaturas principais, que são as curvaturas volumétrica, superficial e linear. Para tal faremos uso da seguinte notação para os elementos da diagonal de $D N$ :

$$
\begin{aligned}
& k_{n_{1}}=\frac{\operatorname{sen} \phi}{2}\left[k_{n_{1}}^{2}(r)-k_{n_{1}}^{1}(r)\right], \\
& k_{n_{2}}=\frac{1}{2 \operatorname{sen} \phi}\left[k_{n_{2}}^{2}(r)-k_{n_{2}}^{1}(r)\right] \mathrm{e} \\
& k_{n_{3}}=\frac{1}{2 \operatorname{sen} \phi}\left[k_{n_{3}}^{2}(r)-k_{n_{3}}^{1}(r)\right] .
\end{aligned}
$$

Para os outros termos usaremos:

$$
\begin{aligned}
\tau_{12} & =\frac{1}{2}\left[\tau_{12}^{2}(r)-\tau_{12}^{1}(r)\right], \\
\tau_{13} & =\frac{1}{2}\left[\tau_{13}^{2}(r)-\tau_{13}^{1}(r)\right] \mathrm{e} \\
\tau_{23} & =\frac{1}{2 \operatorname{sen} \phi}\left[\tau_{23}^{2}(r)-\tau_{23}^{1}(r)\right] .
\end{aligned}
$$


Então temos que a curvatura volumétrica é dada por:

$$
\mathcal{K}=\operatorname{det}(-D N)=-\left[k_{n_{1}} k_{n_{2}} k_{n_{3}}+2 \tau_{12} \tau_{13} \tau_{23}-k_{n_{1}}\left(\tau_{23}\right)^{2}-k_{n_{2}}\left(\tau_{13}\right)^{2}-k_{n_{3}}\left(\tau_{12}\right)^{2}\right] .
$$

A curvatura linear é dada por:

$$
\mathcal{H}=\operatorname{traço~}(-D N)=-\frac{1}{3}\left(k_{n_{1}}+k_{n_{2}}+k_{n_{3}}\right) .
$$

A curvatura superficial é dada por:

$$
\mathcal{H}_{1}=\frac{1}{2}\left\{\left[k_{n_{1}} k_{n_{2}}-\left(\tau_{12}\right)^{2}\right]+\left[k_{n_{1}} k_{n_{3}}-\left(\tau_{13}\right)^{2}\right]+\left[k_{n_{2}} k_{n_{3}}-\left(\tau_{23}\right)^{2}\right]\right\} .
$$

Deixamos para estudos futuros expressar as funções simétricas elementares em função das funções simétricas elementares das hipersuperfícies $B_{i}$. Além disso, gostaríamos de encontrar uma lei de formação para estas funções quando a dimensão for aumentando, tendo como modelo e ponto de partida o caso das curvas no plano e superfícies no $\mathbb{R}^{3}$, apresentado no capítulo 1 . Um aprimoramento pertinente consiste em reduzir o número de ângulos nas expressões acima para o número de 6 . 


\section{Referências Bibliográficas}

[1] M. do Carmo, Differential Geometry of Curves and Surfaces, Prentice-Hall, (1976).

[2] G. Darboux, Sur la forme des lignes de courbure dans la voisinage d'un ombilic, Lecons sur la Theorie des Surfaces, IV, Note 7, Gauthier Villars, Paris, (1896).

[3] A. R. Forsyth, Geometry of Four Dimensions, vols. I e II, Cambridge Univ. Press, Cambridge, (1930).

[4] R. Garcia, J.Sotomayor, Lectures on the Qualitative Theory of Differential Equations of Classical Geometry, pré-publicação UFG.

[5] A. Gray, Modern Differential Geometry de Curves and Surfaces with Mathematica, CRC Press, 2 edição, (1997).

[6] N. Hicks, Notes on Differential Geometry, McGraw-Hill, (1963).

[7] A. Okabe, B. Boots, K. Sugihara, Spatial Tesselations: Concepts and Applications of Voronoi Diagrams, Wiley Ser. Probab. Math. Statist. Appl. Probab. Statist., Wiley, Chichester, (1992).

[8] B. O'Neil, Elementary Differential Geometry, Ac. Press, (1966).

[9] I. R. Porteous. Geometric Differentiation for the Intelligence of Curves and Surfaces, Cambridge Univ. Press, Cambridge, (1994).

[10] L. Rodríguez, Geometria das Subvariedades, Monografias de Matemática, 26, IMPA, (1976).

[11] D. Siersma, Properties of conflict sets in the plano, Caustics 98, Banach Center Publications, vol. 50, Polish Acad. Sciences, Warszawa, (1999).

[12] J. Sotomayor, Lições de Equações Diferenciais Ordinárias, Projeto Euclides, CNPq, (1979). 
[13] J. Sotomayor, R. Garcia, D. Siersma, Curvatures of Conflict Surfaces in Euclidean 3-Space, Caustics 98, Banach Center Publications, vol. 50, Polish Acad. Sciences, Warszawa, (1999).

[14] J. Sotomayor, C. Gutierrez, Lines of Curvature, Umbitic Points and Carathéodory Conjecture, Resenhas IME-USP, 3:291-322, (1998).

[15] J. Sotomayor, C. Gutiérrez, Lines of Curvature and Umbilic Points on Surfaces, Colóquio Bras. de Mat. IMPA, (1991).

[16] J. Sotomayor, C. Gutiérrez, Structurally stable configurations of lines of principal curvature, Astérisque 98-99, (1982), 195-215.

[17] M. Spivak, A Comprehensive Introduction to Differential Geometry, vols. I e III, Publish or Perish, Wilmington, (1979).

[18] D. J. Struik, Lectures on Classical Differential Geometry, Addison Wesley, 1950, Reprinted by Dover, New York, (1988). 